\title{
Proceedings of the Cybersecurity for Direct Digital Manufacturing (DDM) Symposium
}

Celia Paulsen

This publication is available free of charge from: http://dx.doi.org/10.6028/NIST.IR.8041 


\section{Proceedings of the Cybersecurity for Direct Digital Manufacturing (DDM) Symposium}

Celia Paulsen

Computer Security Division Information Technology Laboratory

This publication is available free of charge from: http://dx.doi.org/10.6028/NIST.IR.8041

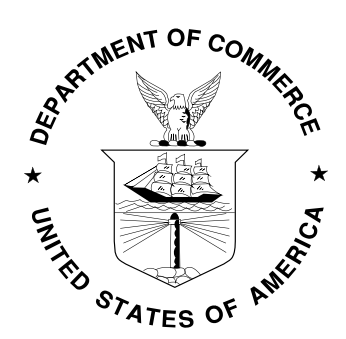

U.S. Department of Commerce

Penny Pritzker, Secretary

National Institute of Standards and Technology Willie May, Acting Under Secretary of Commerce for Standards and Technology and Acting Director 


\title{
National Institute of Standards and Technology Internal Report 8041 143 pages (April 2015)
}

This publication is available free of charge from: http://dx.doi.org/10.6028/NIST.IR.8041

Certain commercial entities, equipment, or materials may be identified in this document in order to describe an experimental procedure or concept adequately. Such identification is not intended to imply recommendation or endorsement by NIST, nor is it intended to imply that the entities, materials, or equipment are necessarily the best available for the purpose.

There may be references in this publication to other publications currently under development by NIST in accordance with its assigned statutory responsibilities. The information in this publication, including concepts and methodologies, may be used by Federal agencies even before the completion of such companion publications. Thus, until each publication is completed, current requirements, guidelines, and procedures, where they exist, remain operative. For planning and transition purposes, Federal agencies may wish to closely follow the development of these new publications by NIST.

Organizations are encouraged to review all draft publications during public comment periods and provide feedback to NIST. All NIST Computer Security Division publications, other than the ones noted above, are available at http://csrc.nist.gov/publications.

\section{Comments on this publication may be submitted to:}

\author{
National Institute of Standards and Technology \\ Attn: Computer Security Division, Information Technology Laboratory \\ 100 Bureau Drive (Mail Stop 8930) Gaithersburg, MD 20899-8930 \\ Email: csddm@nist.gov
}




\title{
Reports on Computer Systems Technology
}

The Information Technology Laboratory (ITL) at the National Institute of Standards and Technology (NIST) promotes the U.S. economy and public welfare by providing technical leadership for the Nation's measurement and standards infrastructure. ITL develops tests, test methods, reference data, proof of concept implementations, and technical analyses to advance the development and productive use of information technology. ITL's responsibilities include the development of management, administrative, technical, and physical standards and guidelines for the cost-effective security and privacy of other than national security-related information in Federal information systems.

\begin{abstract}
Direct Digital Manufacturing (DDM) involves fabricating physical objects from a data file using computer-controlled processes with little to no human intervention. It includes Additive Manufacturing (AM), 3D printing, and rapid prototyping. The technology is advancing rapidly and has the potential to significantly change traditional manufacturing and supply chain industries, including for information and communication technologies (ICT).

On February 3, 2015, the National Institute of Standards and Technology (NIST) Information Technology Laboratory (ITL) Computer Security Division hosted a one-day symposium to explore cybersecurity needed for DDM, to include ensuring the protection of intellectual property and the integrity of printers, elements being printed, and design data. Speakers and attendees from industry, academia, and government discussed the state of the industry, cybersecurity risks and solutions, and implications for Information and Communications Technology (ICT) supply chain risk management.
\end{abstract}

\section{Keywords}

3D Printing; Additive Manufacturing; Cyber Physical Systems; Cybersecurity; Direct Digital Manufacturing; Industrial Control Systems; Information Security 


\section{Acknowledgements}

The NIST Information Technology Laboratory would like to acknowledge Kevin Jurrens, Richard Ricker, Kim Schaffer, and Bill Newhouse of NIST for their contributions in putting together this symposium. NIST would also like to acknowledge each of the presenters for their participation. 


\section{Executive Summary}

Information Technology has increasingly been incorporated into every segment of the economy. In manufacturing, the basic technology of Direct Digital Manufacturing (DDM) been around for dozens of years. This involves the creation of a physical object from a digital design using computer-controlled processes with little to no human intervention. With the popularization and advancement of Additive Manufacturing (AM) and $3 \mathrm{D}$ printing, it is becoming much more common. These technologies have the potential to significantly change traditional manufacturing and supply chain industries, including information and communications technologies (ICT).

On February $3^{\text {rd }}, 2015$, the NIST Information Technology Laboratory (ITL) Computer Security Division hosted a one-day symposium to explore the cybersecurity aspects of DDM. There were approximately 50 attendees from government, industry, and academia representing a broad array of DDM practitioners, cybersecurity professionals, researchers, and manufacturing innovation organizations.

During the symposium, speakers and attendees discussed DDM cybersecurity risks, challenges, solutions, and implications for ICT supply chain risk management. Although the presenters were all from diverse backgrounds representing a variety of viewpoints, each had similar arguments:

- Cybersecurity risks to DDM are very real;

- Cybersecurity threats have the potential to disrupt the manufacturing revolution;

- There is real opportunity to improve the security of the manufacturing supply chain, and

- The time to build cybersecurity in to the DDM process is now.

During discussions and the concluding working session, participants generally agreed that the biggest challenge to building cybersecurity into DDM is culture. Organizations especially small businesses - may not recognize that AM or 3D printing devices have any cybersecurity risks and may be unwilling to compromise efficiency for security. Other key areas discussed included cost-effective technological capabilities, technical standards, and general guidance. While several existing technical standards were identified, most were not specific to cybersecurity in DDM. Attendees noted that technical and standards-based solutions for DDM are limited and do not address the rapid, changeable, and distributed manufacturing environment of the future. NIST SP 800-53 Revision 4, Security and Privacy Controls for Federal Information Systems and Organizations[1], and the NIST Framework for Improving Critical Infrastructure Cybersecurity[2] were identified as potential starting points for developing risk management guidance for DDM. 


\section{Table of Contents}

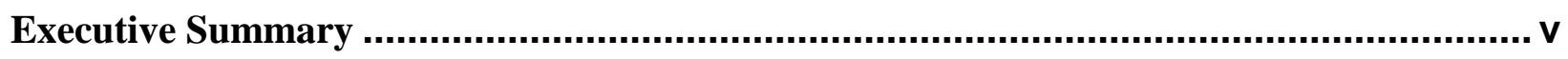

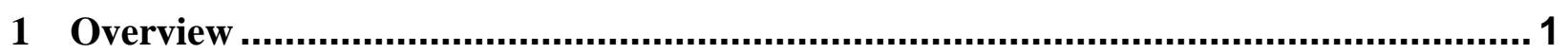

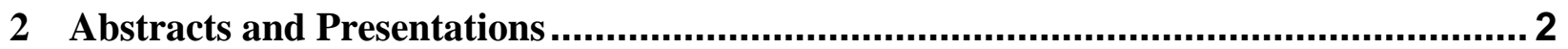

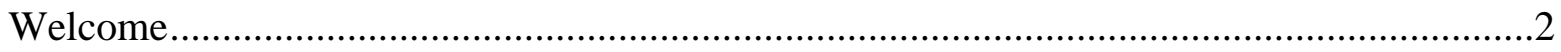

James St. Pierre

Deputy Director of the Information Technology Laboratory (ITL), NIST

Invited Talk

Michael F. Molnar

Director, NIST Advanced Manufacturing Program Office

Director, Advanced Manufacturing National Program Office (AMNPO)

Presentation

Presentation 1: An Analysis of Cyber Physical Vulnerabilities in Additive

Manufacturing

Christopher B. Williams

Associate Professor, Virginia Tech Department of Mechanical Engineering

Abstract. .20

Presentation

Presentation 2: Applying and Assessing Cybersecurity Controls for Direct Digital

Manufacturing Systems

Scott Zimmerman, CISSP-ISSEP

Principal IT Advisor, Concurrent Technologies Corporation (CTC)

Dominick Glavach, CISSP, GCIH

Principle Fellow, Information Systems Security Engineer, CTC

Abstract .52

Presentation

Presentation 3: Cybersecurity for Advanced Manufacturing - Securing the Digital

Thread

Dr. Michael F. McGrath

NDIA Manufacturing Division

Abstract .66

Presentation

Panel: Opportunities for Secure 3D Printing .......

Robert Zollo (moderator)

President, Avante Technology 
Abstract.....

Presentation

Dr. Claire Vishik

Trust and Security Technology and Policy Director, Intel Corporation

Presentation

Andre Wegner

Founder, CEO at Authentize

Presentation

3 Summary of Attendee Perceptions .................................................................. 118

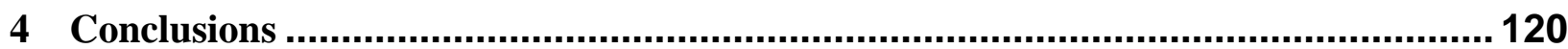

\section{List of Appendices}

Appendix A- Response Sheet Results .................................................. A-1

Appendix B-Working Session Results ...................................................... B-1

Appendix C- Biographies ............................................................................ C-1

Appendix D- Attendee List ..................................................................... D-1

Appendix E-Acronyms ..................................................................................E-1

Appendix F- References .................................................................................F-1 


\section{Overview}

Direct Digital Manufacturing (DDM) involves fabricating physical objects from a data file using computer-controlled processes with little to no human intervention. Traditionally, these technologies have not been widely adopted, but with the popularization of Additive Manufacturing (AM) and 3D printing, they are becoming increasingly common. These technologies are advancing rapidly and have the potential to significantly change traditional manufacturing and supply chain industries, including for information and communication technologies (ICT).

On February 3, 2015, the National Institute of Standards and Technology (NIST) Information Technology Laboratory (ITL) Computer Security Division hosted a one-day symposium to explore the cybersecurity aspects of DDM, to include ensuring the protection of intellectual property and the integrity of printers, elements being printed, and design data.

There were approximately 50 attendees from government, industry, and academia representing a broad array of DDM practitioners, cybersecurity professionals, researchers, and manufacturing innovation organizations. During the symposium, speakers and attendees discussed cybersecurity risks, challenges, solutions, and implications for Information and Communications Technology (ICT) supply chain risk management.

The agenda contained an invited talk, four presentations, and a panel discussion that exemplified diverse perspectives. A concluding working session captured the viewpoints of the attendees in several key areas. In addition, attendees provided inputs on the risks, challenges, existing solutions, and potential/theoretical solutions for cybersecurity in DDM. Responses focused around culture / humans, threats to the integrity of design, technological capabilities - especially around quality control and event detection, and guidance specific to cybersecurity in DDM.

The remainder of this publication is structured as follows:

- Section 2 contains a summary of each presentation, and speaker submitted abstracts and presentations where applicable. Presentations are included in the order they were given during the symposium.

- Section 3 contains an analysis of attendee perceptions based on completed attendee handouts / response sheets and the concluding working session.

- Section 4 presents conclusions, including possible future steps and recommendations.

- Appendix A contains data from completed handouts / response sheets.

- Appendix B contains data collected during the concluding working session

- Appendix C contains biographies of the presenters as contained in the agenda.

- Appendix D lists acronyms used throughout the document. 


\section{Abstracts and Presentations}

This section contains a brief summary of each presentation along with the abstracts speakers submitted, when applicable, and any slides used. Presentations in this section are listed in the order they were given during the symposium.

\section{Welcome}

James St. Pierre

Deputy Director of the Information Technology Laboratory (ITL), NIST

Key Points:

- NIST's mission is to promote "U.S. innovation and industrial competitiveness."

- Safeguarding the "digital threads" of the manufacturing process is critical to promoting innovation and industrial competitiveness.

- The core principles of NIST's ITL efforts include collaboration, openness, and transparency.

- We welcome the opportunity to collaborate to identify risks, challenges, gaps and opportunities as we look to "build security in" to the direct digital manufacturing processes and discuss ways forward.

\section{Invited Talk}

Michael F. Molnar

Director, NIST Advanced Manufacturing Program Office

Director, Advanced Manufacturing National Program Office (AMNPO)

Key Points:

- The first two manufacturing revolutions were about bringing capabilities together. The third and current manufacturing revolution is about new capabilities - creating things we never could have before.

- Misconceptions about manufacturing include that it is "dirty and declining," meaning it may not be an attractive job field.

- Manufacturing plays a central role in the U.S. economic base.

- In 2013, the National Network of Manufacturing Innovation (NNMI) was created with bi-partisan support to advance the US's manufacturing capabilities.

- The Revitalize American Manufacturing Innovation (RAMI) Act of 2014 (H.R. 2996/S. 1468) calls for open-topic proposals for creating additional NNMI institutes. Currently 8 are planned with a goal of 45 total. 
- Ed Morris was invited to speak about the first pilot NNMI institute - America Makes. He spoke about how they examined cyber implications and how advanced manufacturing would not exist without the digital component.

- Dean Bartles was invited to speak about the second pilot NNMI institute - the Digital Manufacturing and Design Innovation Institute (DMDII) in Chicago, Illinois. The DMDII focuses on digital design solutions and that cybersecurity ranked among the top five concerns of manufacturing leaders. DMDII Project Call 15-01 is specifically focused on cybersecurity and closes March 20, 2015.

- With digital manufacturing, the U.S. is regaining its focus on manufacturing and raising a new generation of makers. 
Presentation:

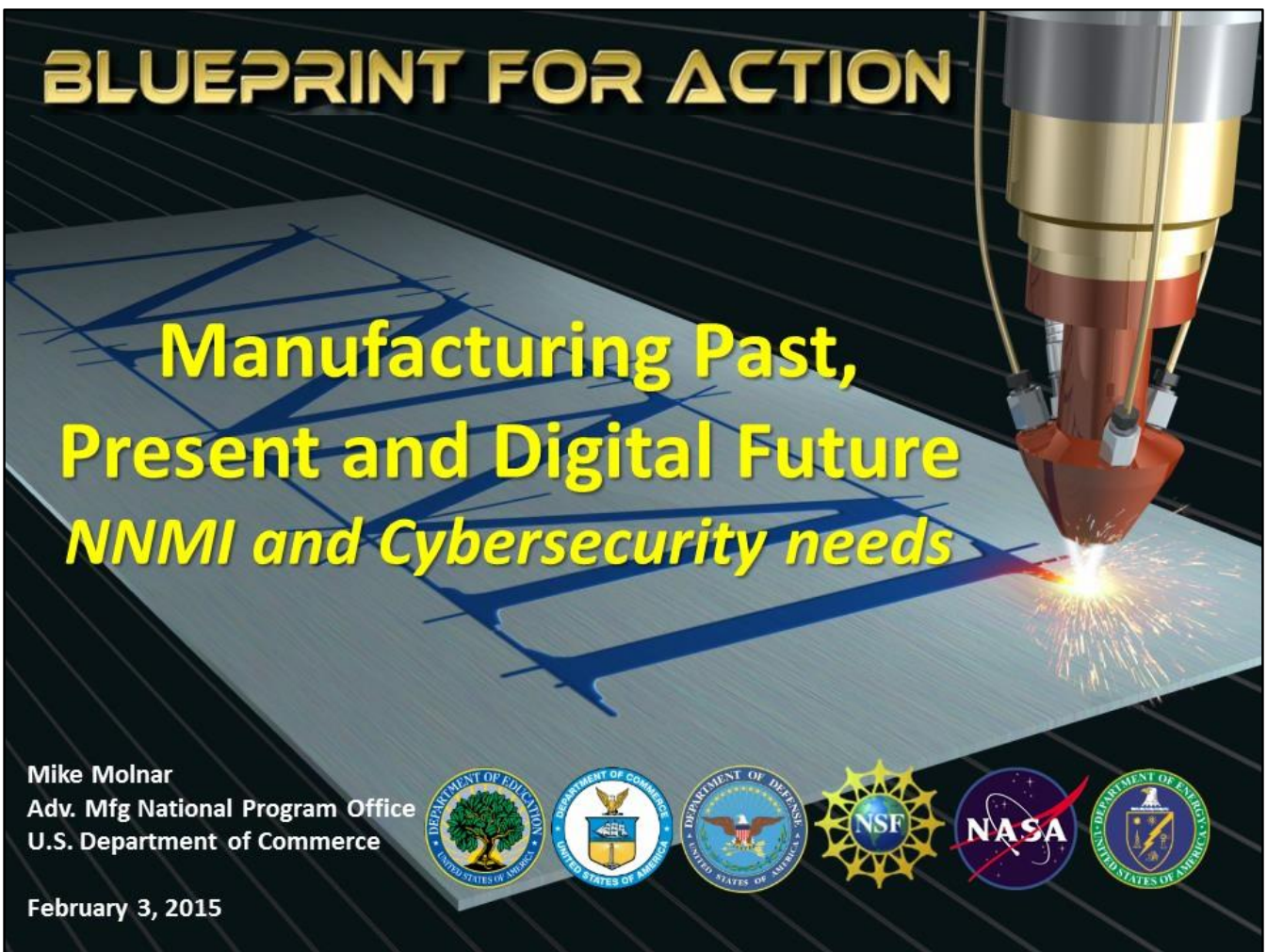

\section{Agenda}

\section{U.S. Manufacturing Yesterday}

Historical View and Challenge

U.S. Manufacturing Today

Creating NNMI

U.S. Manufacturing Tomorrow

A Digital Manufacturing Renaissance requiring Cybersecurity 

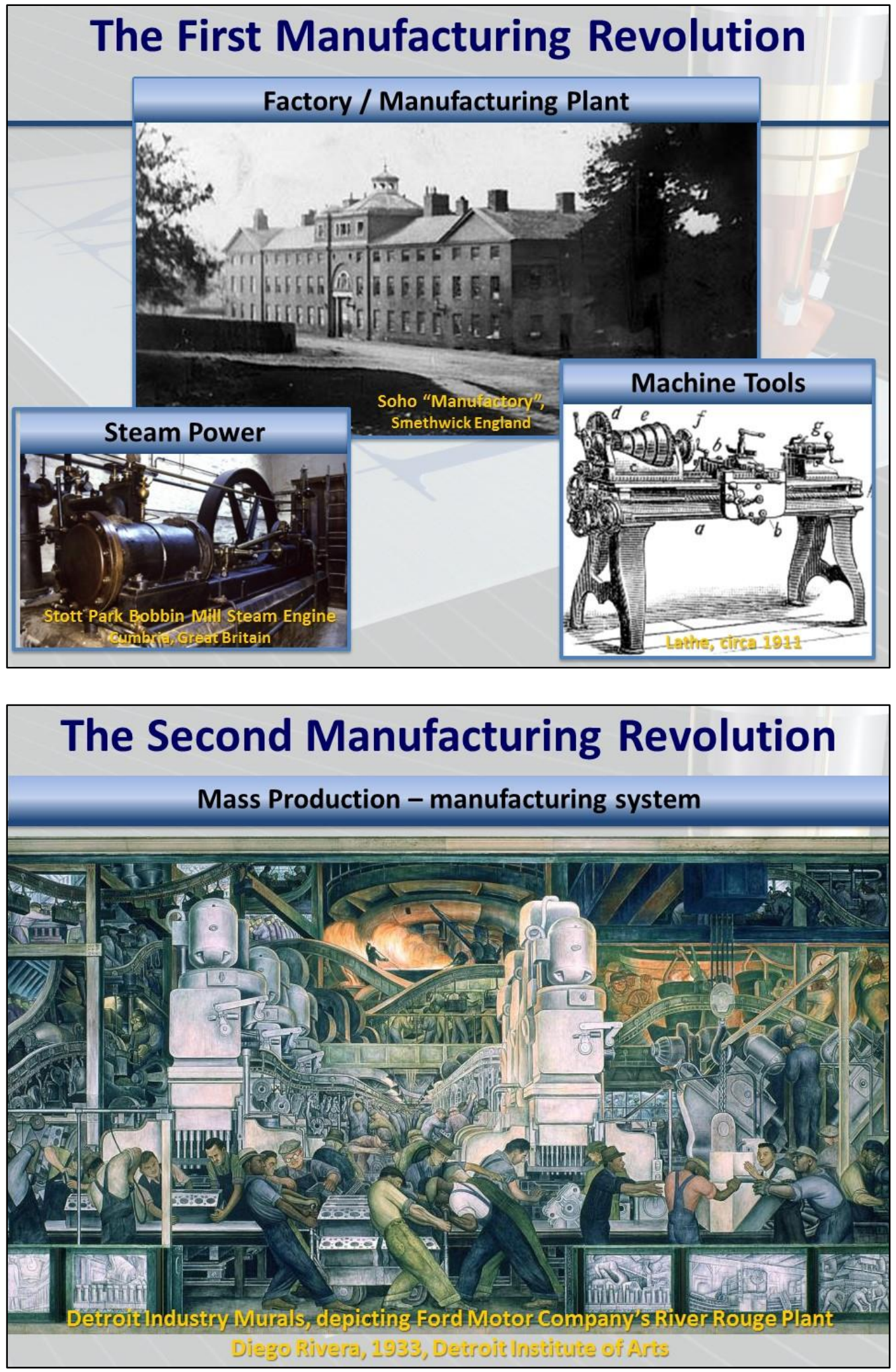


\section{Misperception - Productivity on Employment}

Rising Productivity does not create employment losses $1965-2000$ : US Mfg output rises $\mathbf{6 x}$, stable employment
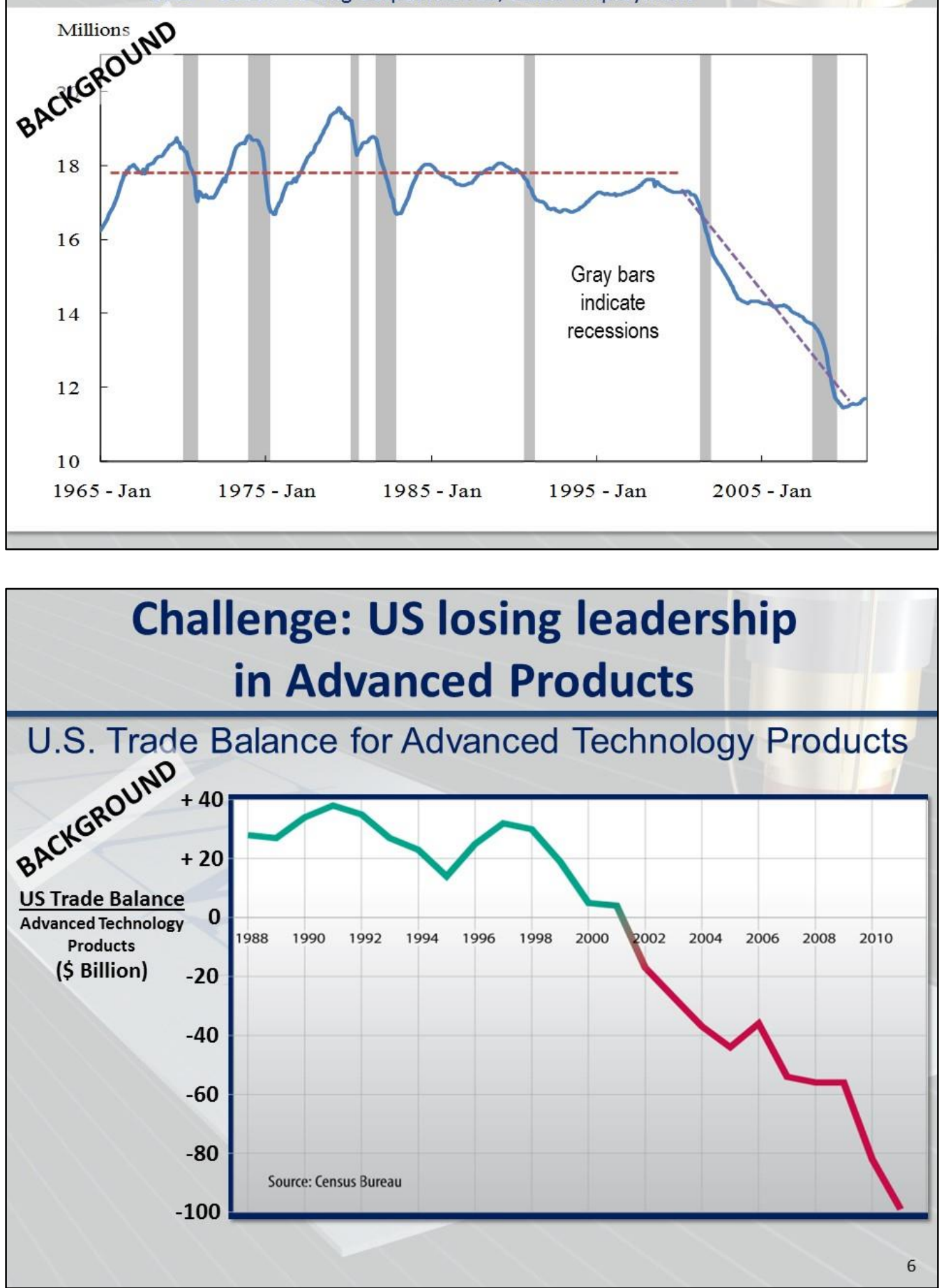


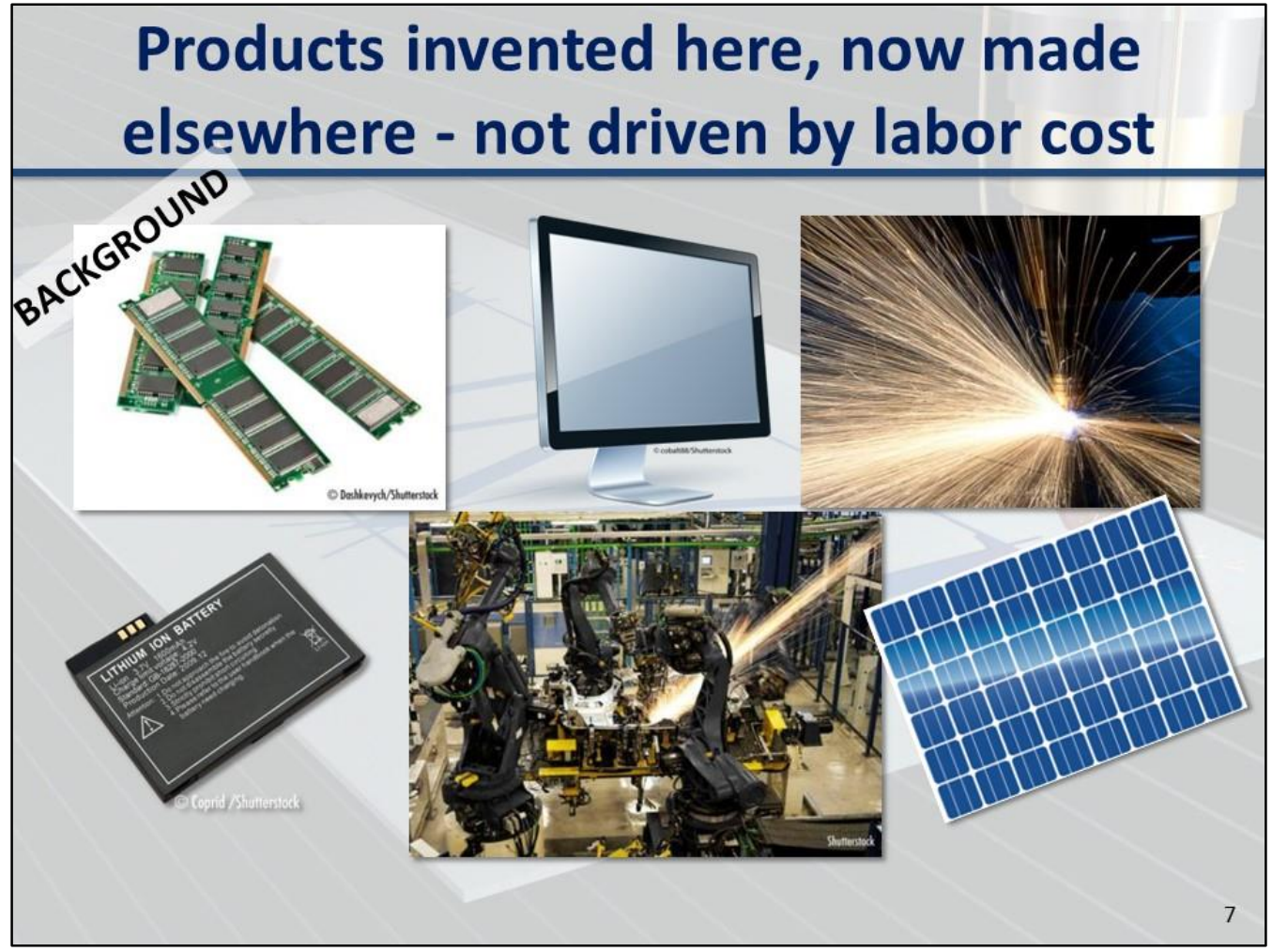

\section{Why should we care about US Manufacturing?}

\section{Critical role in U.S. Innovation Ecosystem}

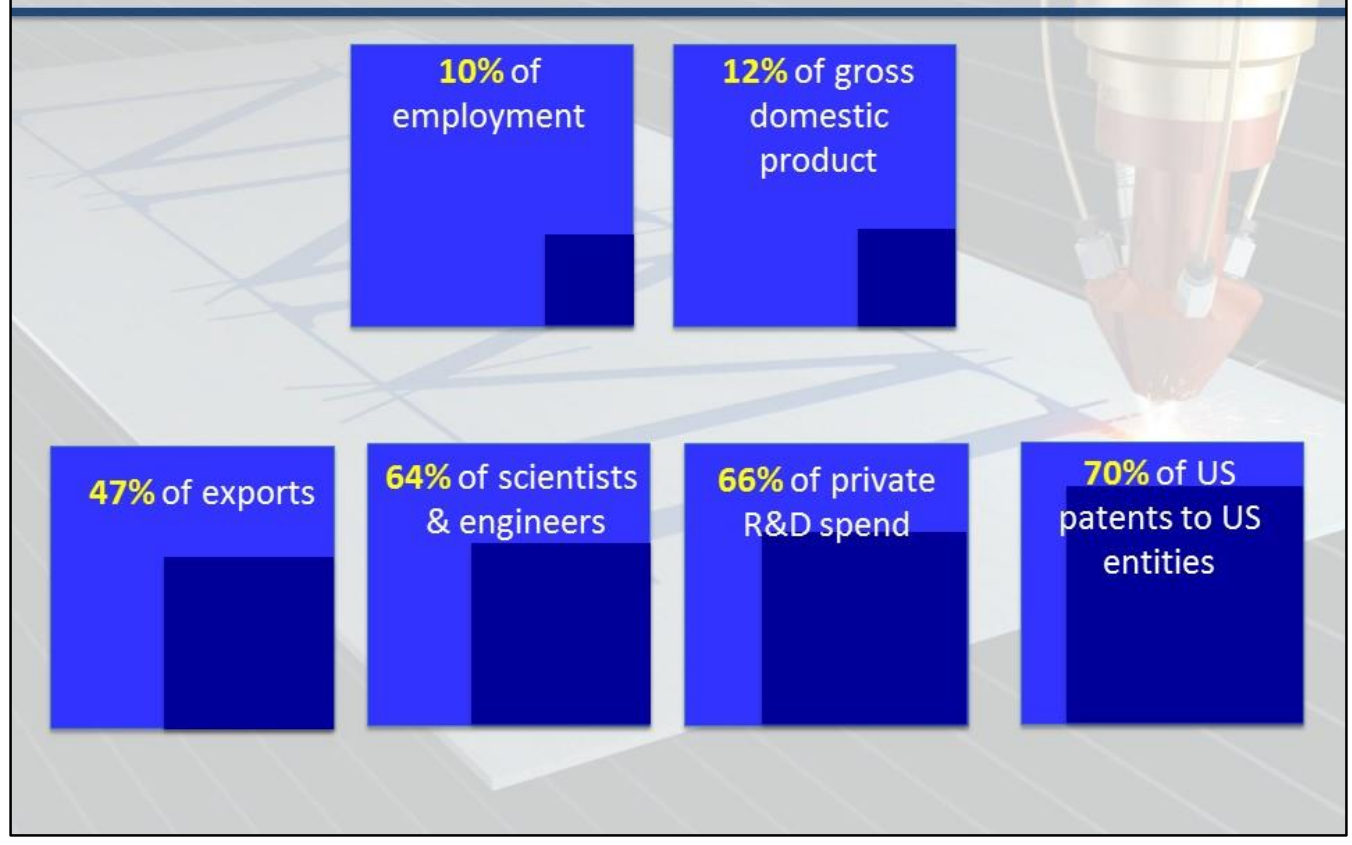




\section{Agenda}

U.S. Manufacturing Yesterday Historical View and Challenge

\section{U.S. Manufacturing Today}

Creating NNMI

U.S. Manufacturing Tomorrow

A Digital Manufacturing Renaissance requiring Cybersecurity

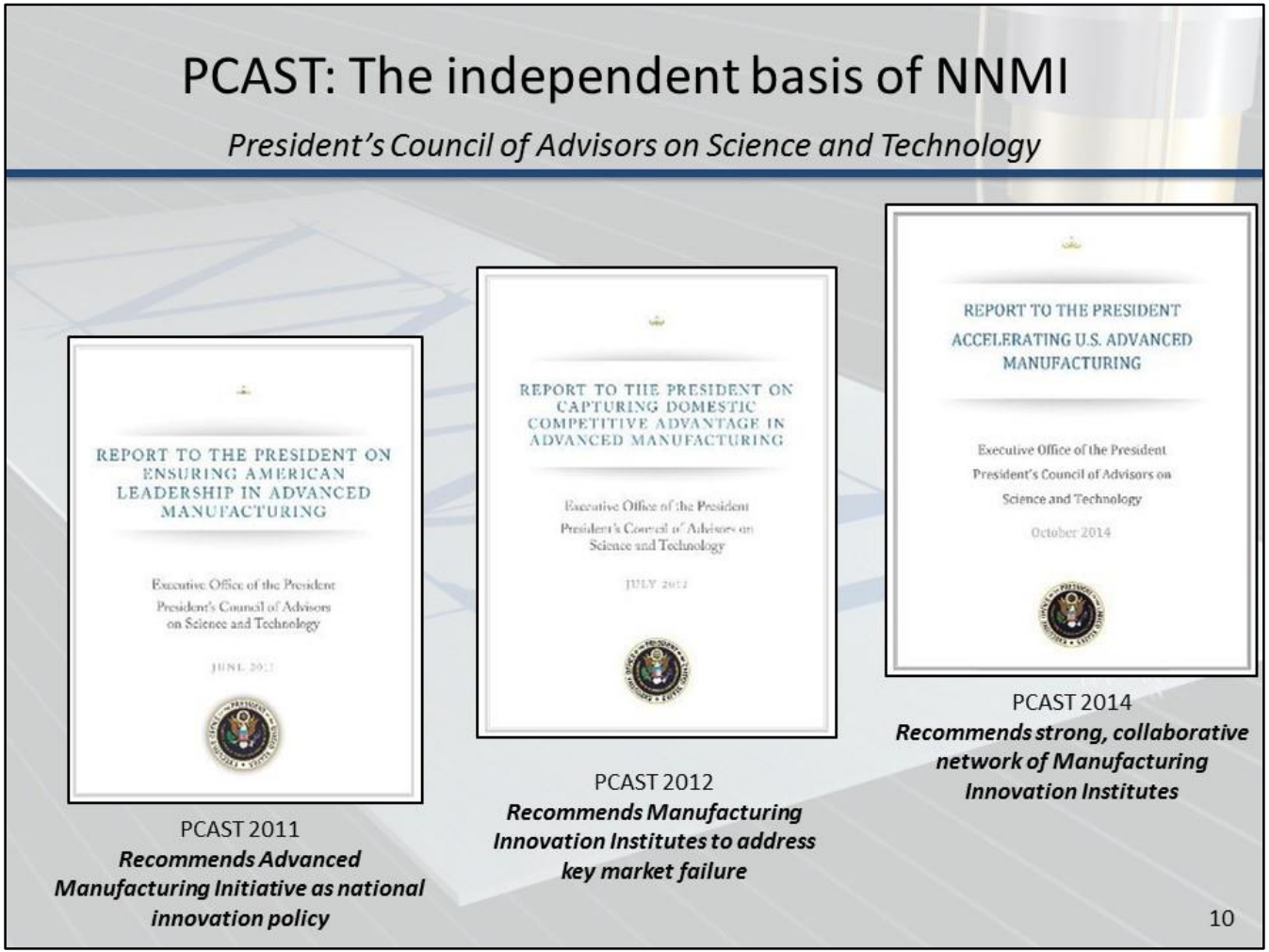



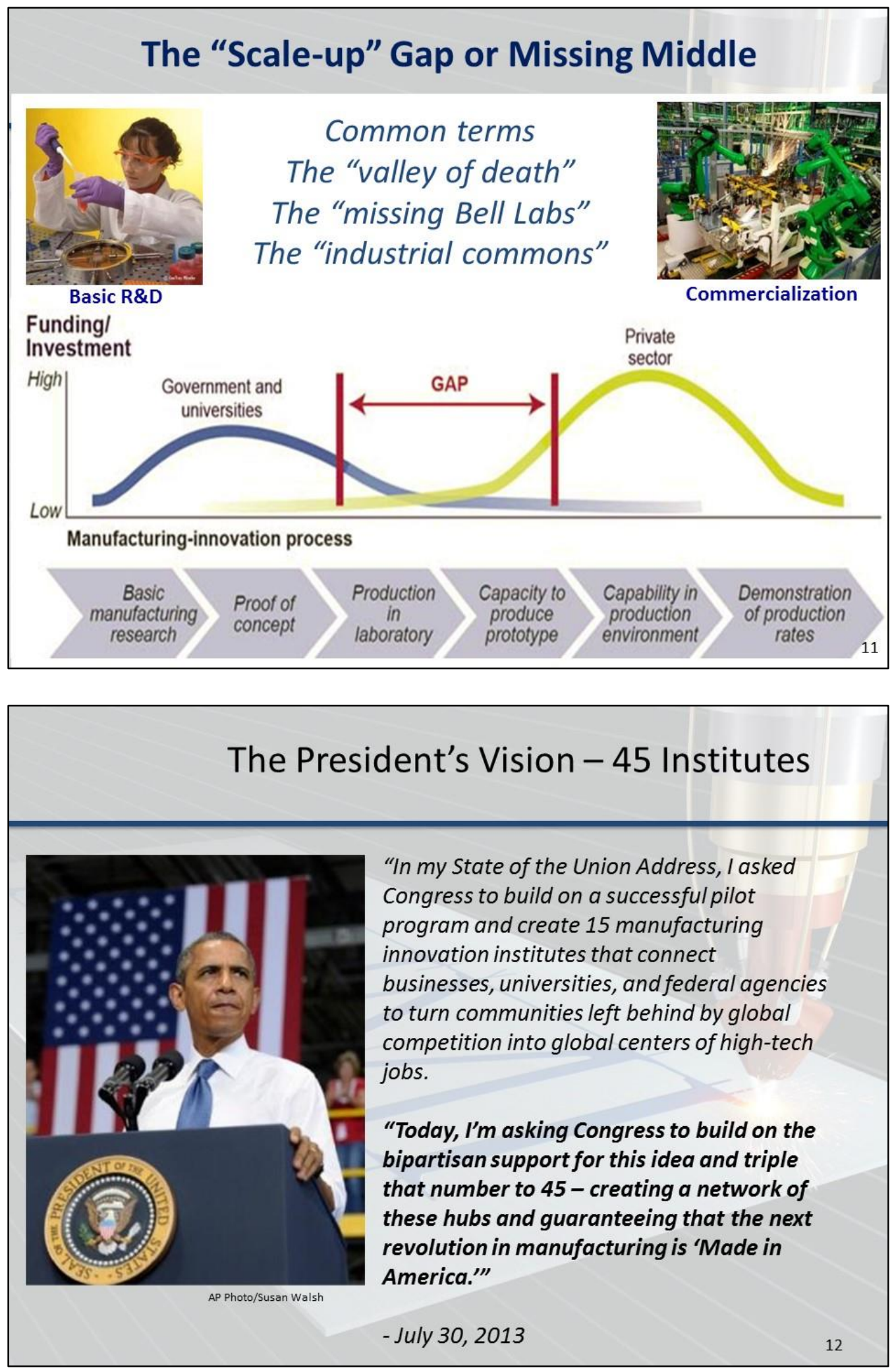

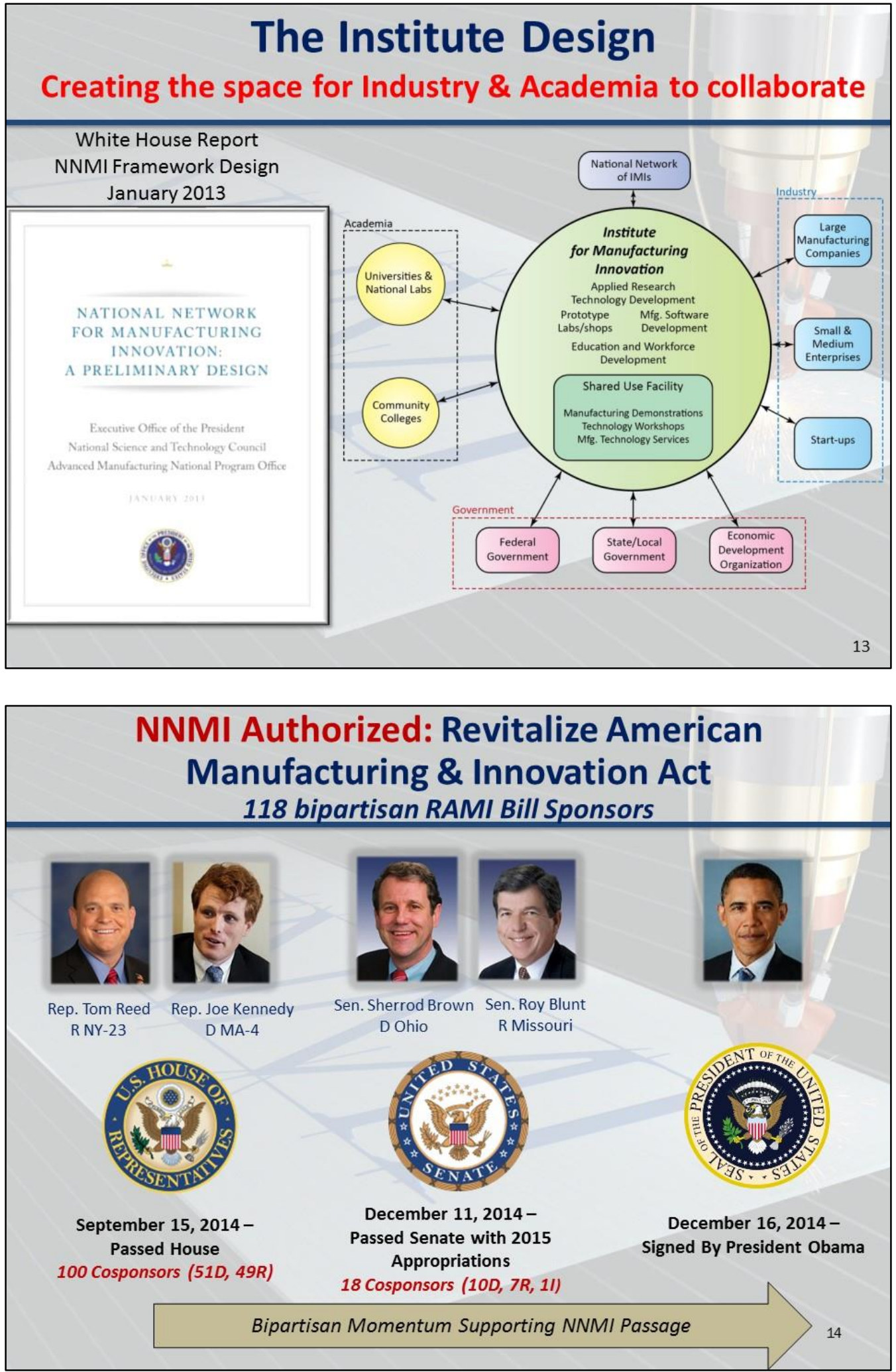


\section{RAMI and NIST}

Call to Action: RAMI calls upon the U.S. Secretary of Commerce and NIST to establish:

1. The "Network for Manufacturing Innovation Program" (Network function) - to convene and support a network of Institutes

2. New "Centers for Manufacturing Innovation" (Institutes) using an open topic, open competition process

3. The National Program Office at NIST - to oversee and carry out the program (coordination, network support, and reporting)

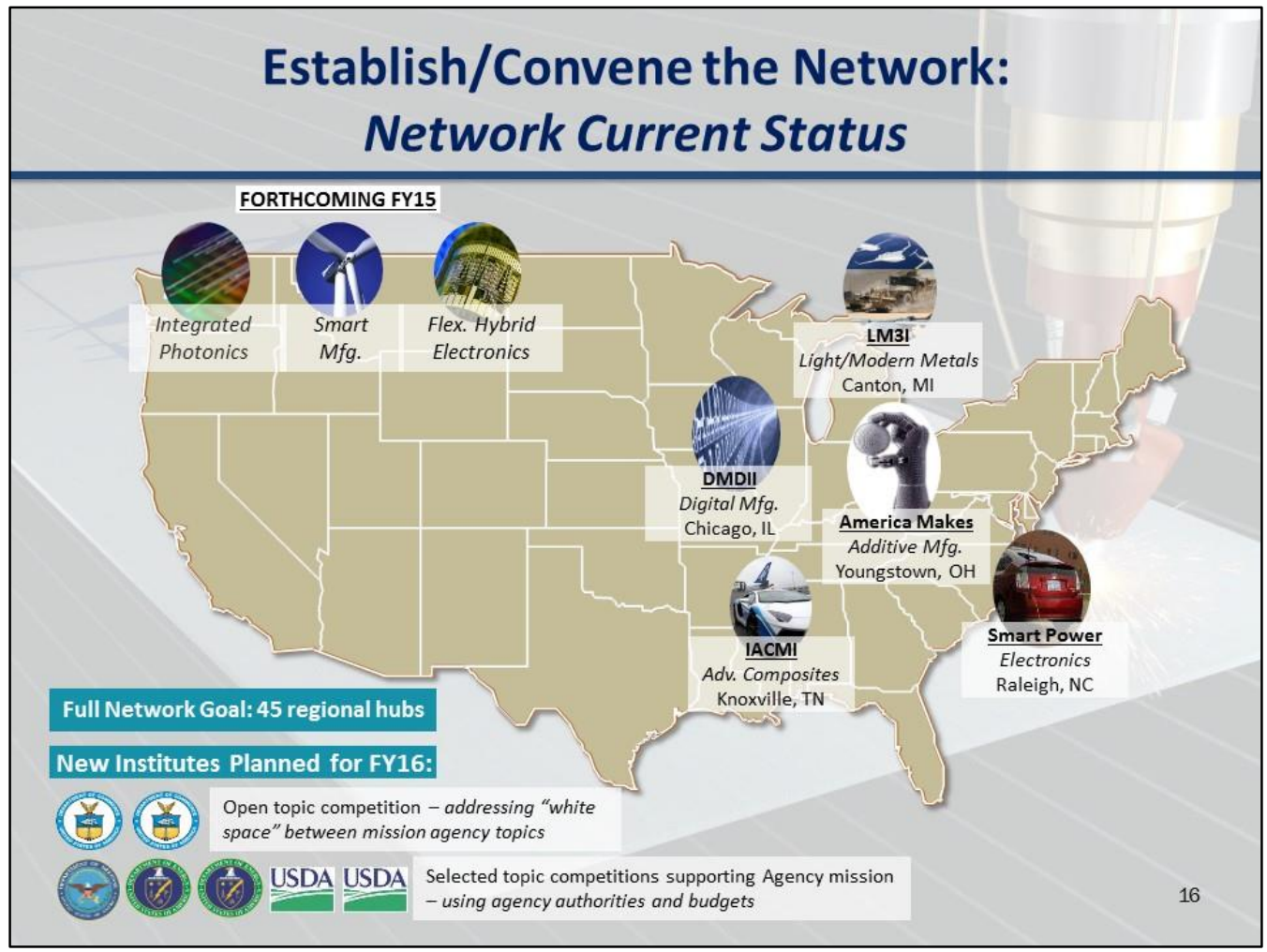




\section{Agenda}

U.S. Manufacturing Yesterday Historical View and Challenge

U.S. Manufacturing Today Creating NNMI

\section{U.S. Manufacturing Tomorrow}

A Digital Manufacturing Renaissance requiring Cybersecurity

\section{The Third Manufacturing Revolution}

Digital Mfg - smart, adaptive, optimized, distributed
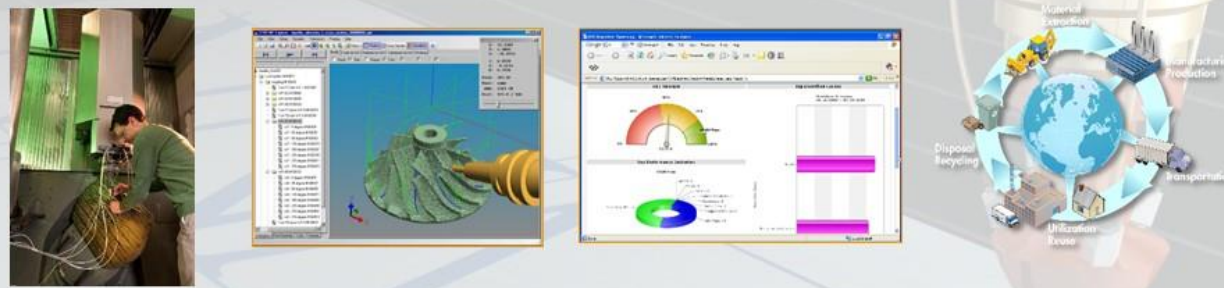

Example Digital Manufacturing Technology - "Additive Manufacturing"
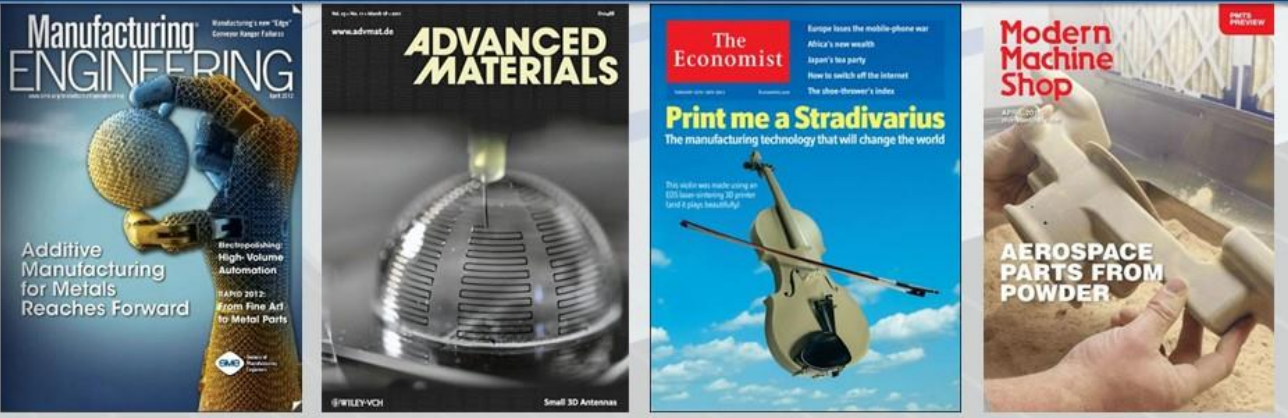


\section{The First Pilot Manufacturing Innovation Institute Additive Manufacturing/3D Printing - Youngstown $\mathrm{OH}$}

Prime Awardee: National Center for Defense Manufacturing and Machining

- Initial \$30M federal investment matched by $\$ 40 \mathrm{M}$ industry, state/local

- Strong leveraging of equipment, existing resources

- Strong business development

- Tiered membership-based model, low cost to small business and nonprofits
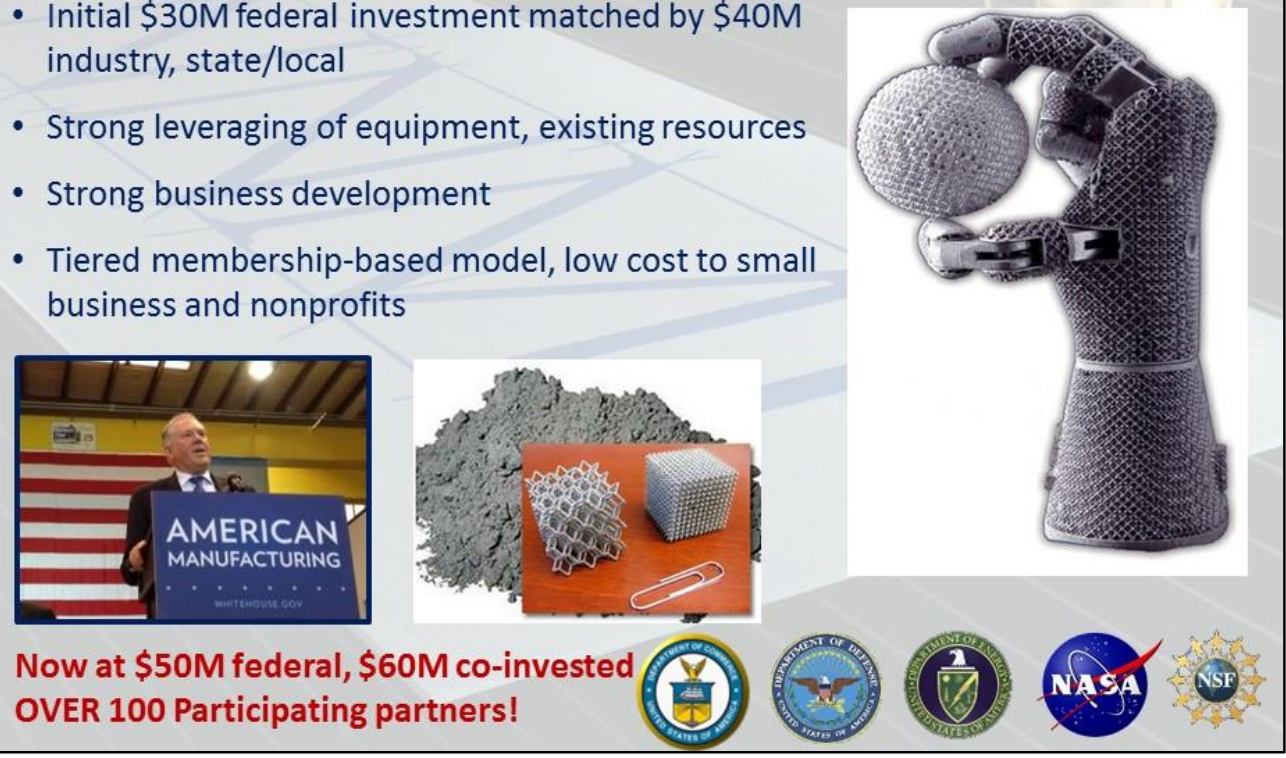

- Now at \$50M federal, \$60M co-invested

- OVER 100 Participating partners!

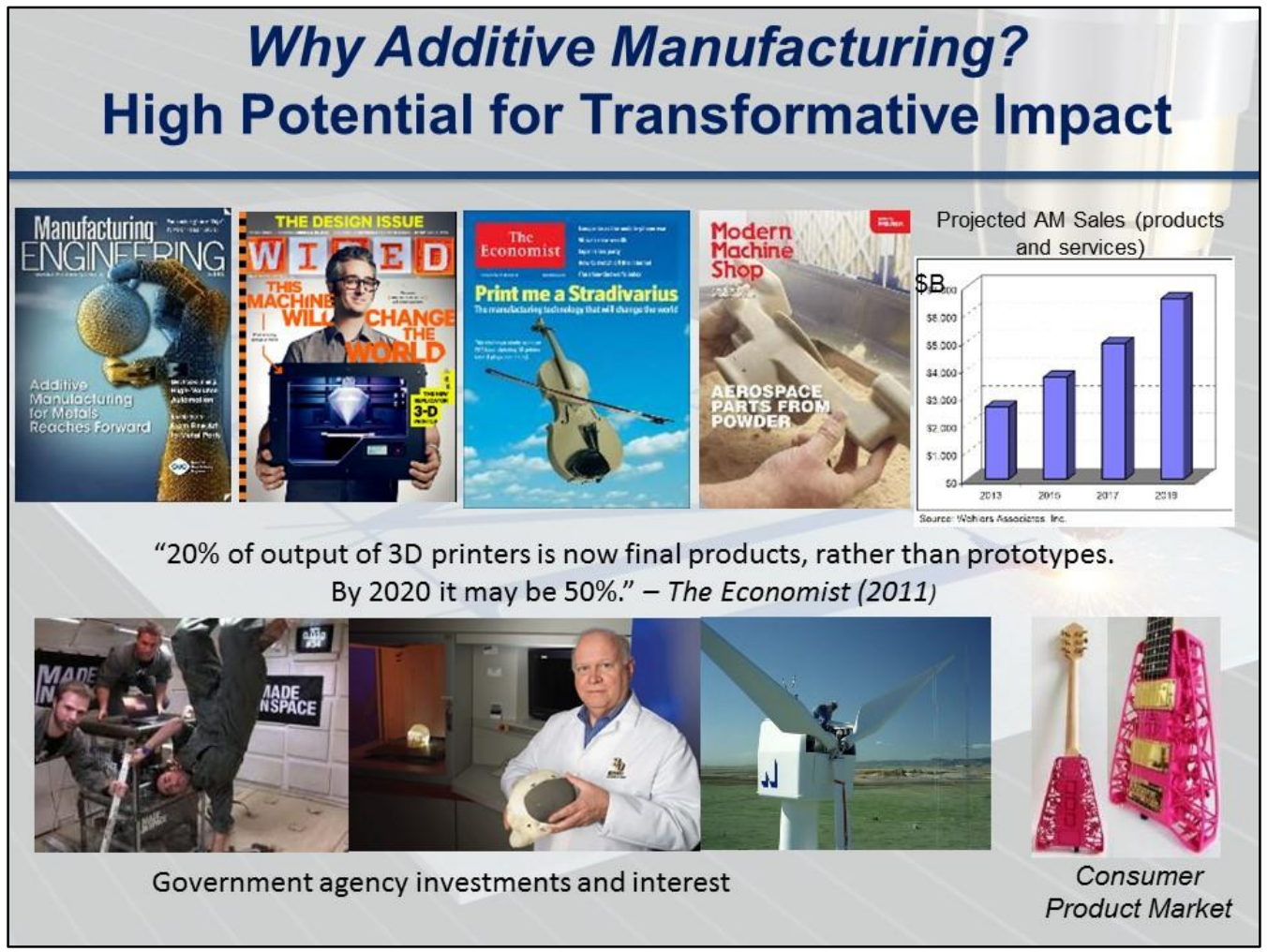




\section{$\triangle$ America Makes}

\section{America Makes - National Additive Manufacturing Innovation Institute: Cybersecurity Actions}

- "Cyber Security Awareness" Presentation to all Members at Spring 2014 Program Management Review by Victoria Yan Pillitteri, Advisor for Information System Security, Computer Security Division, Information Technology Laboratory, National Institute of Standards and Technology

- Reinforced the cybersecurity defenses on our America Makes website after successfully defeating a brute force foreign-based cyber attack in August 2014

- Host periodic FBI Cybersecurity Briefs to America Makes management team

- Deployed Siemens Teamcenter for America Makes PLM data management, hosted by Amazon in "GovCloud"

- Rigorous Password access protocols in place

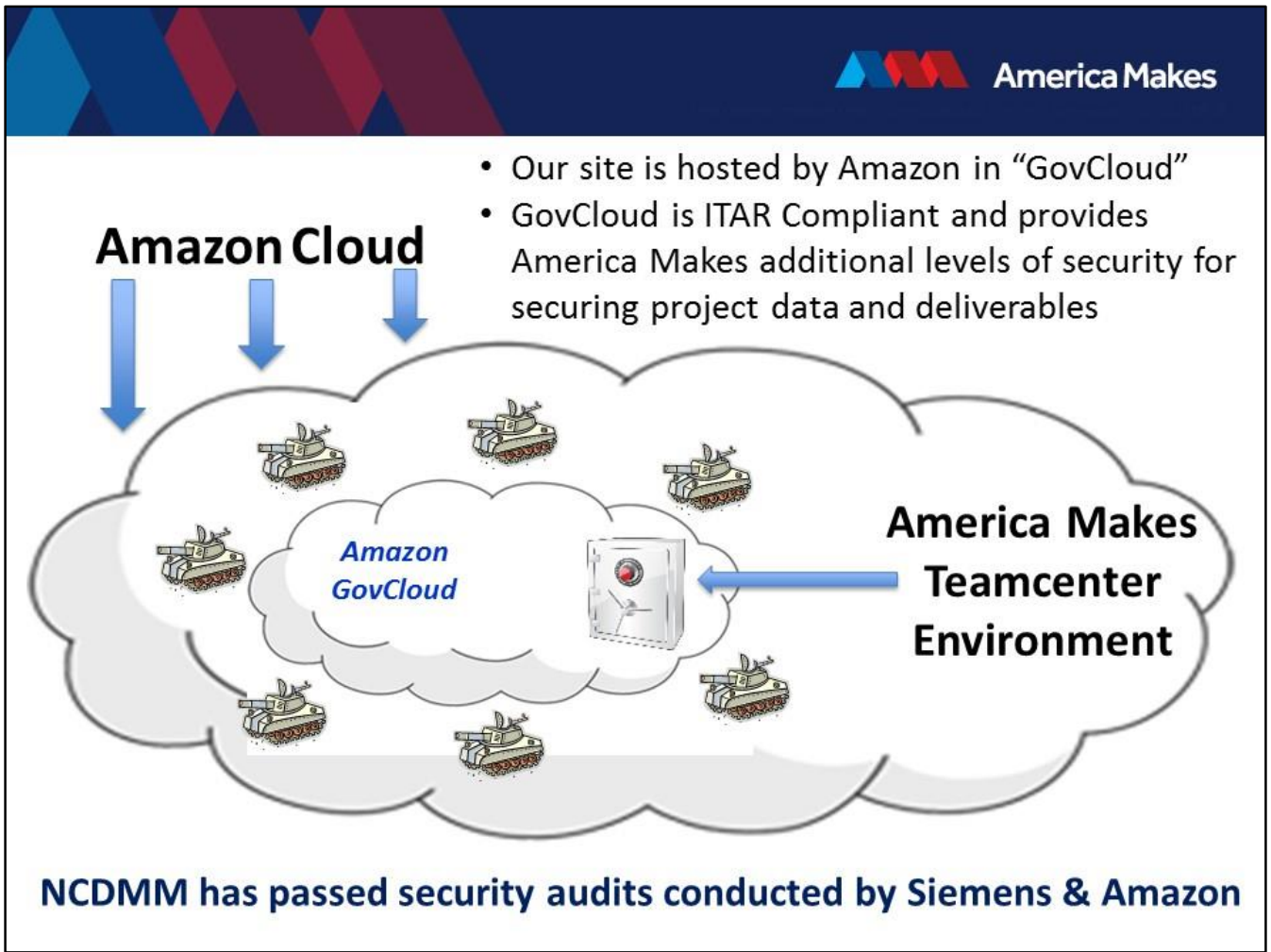



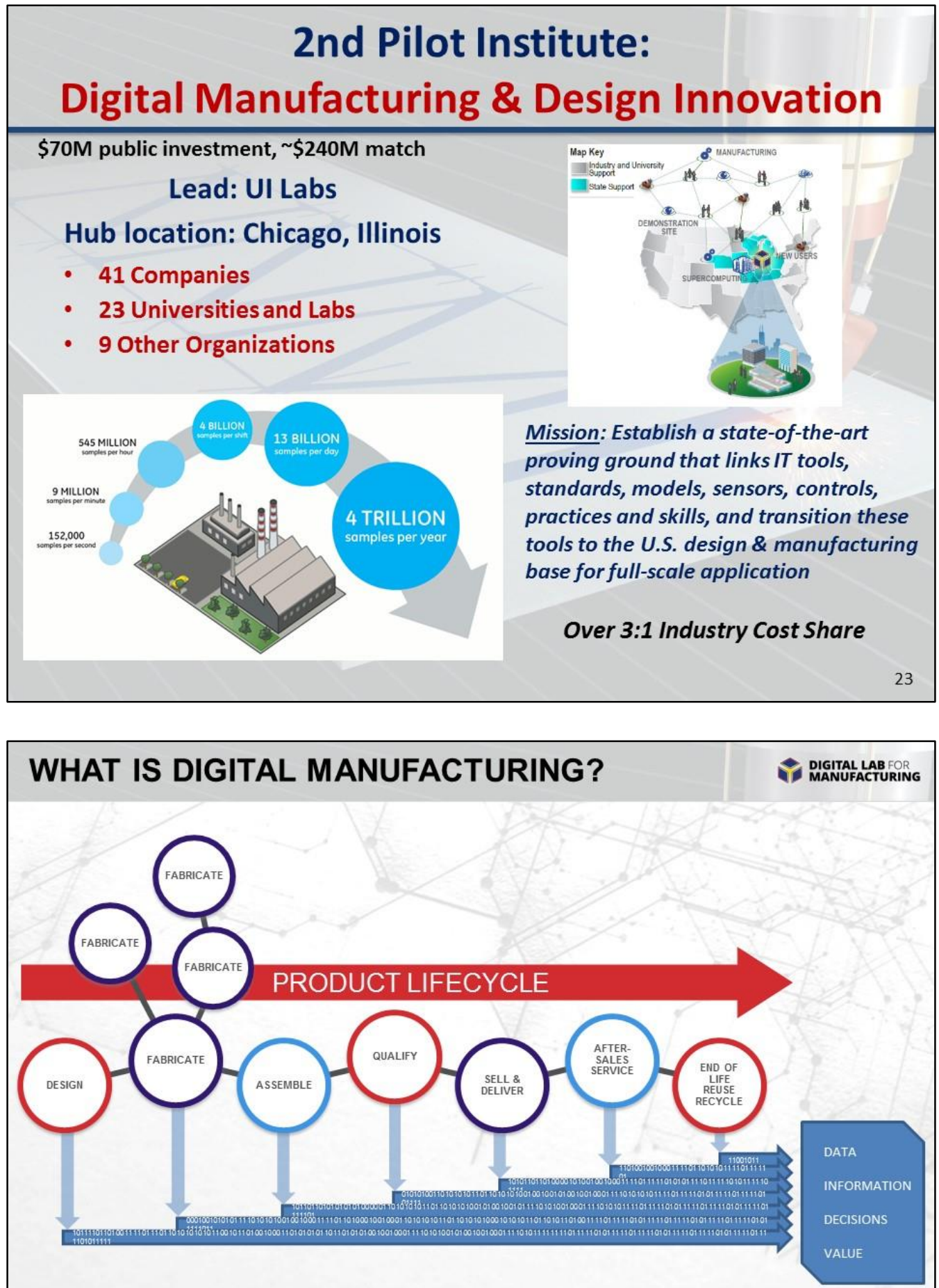

Data is gathered and available for optimization of process and product at every point in the cycle, is aggregated for used in the DIGITAL MANUFACTURING COMMONS 


\section{Manufacturing cyber security ranks among the top five priorities for manufacturing leaders}

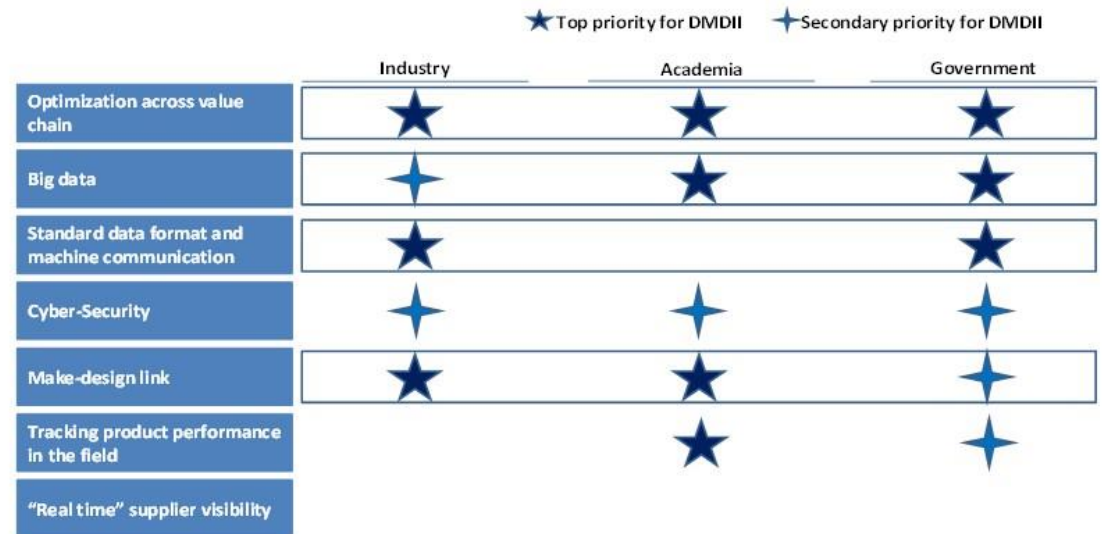

In building the DMDII Technology Roadmap, DMDII surveyed 170 manufacturing leaders from industry, academia, and government.

\section{DMDII Strategic Investment Plan for Intelligent Machines}

\section{Four key DMDII investment areas for 2015 and beyond.}

IM1: Communications Standards for Intelligent Machines

Provide a framework and standards for communication for Intelligent Machines, including: legacy and modern production machines, robotic devices, manufacturing cells, and other smart manufacturing systems.

IM2: Cyber Security for Intelligent Machines

Demonstrate technologies that de-risk the networking of production equipment within the smart factory.

IM3: Operating System for Cyber Physical Manufacturing Technologies for horizontal and vertical resource management from the lowest hardware to the highest enterprise level. Includes real time streaming of machine tool data, and remote operation of production equipment.

IM4: Intelligent Machining Toolkit Use real-time analytics and machine learning to optimize the smart factory. 


\section{DMDII Project Call 15-01: Factory Infrastructure Cybersecurity Assessment}

Key Challenge: Protecting the operational systems of a manufacturing enterprise presents a different set of challenges from protecting enterprise IT systems and networks.

- The project objectives are to:

- Identify minimum capabilities that satisfy DFARS requirements (252.204-7012) for incorporating information security measures in a typical industry setting

- Estimation of the costs to reach and maintain those DFARS-compliant capabilities

- Development and test of a vulnerability assessment tool

White Papers are Due March 20 DMDII will fund 3 projects at a total of $\$ 1.2 \mathrm{M}$ More information at: http://dmdii.uilabs.org

\section{(6), DMDII}

cyber Security

Approved for Public Releas

+ a UI LABS Collaboration

\section{Opportunities from Digital Manufacturing}

Democratization of tools needed to Design and Make
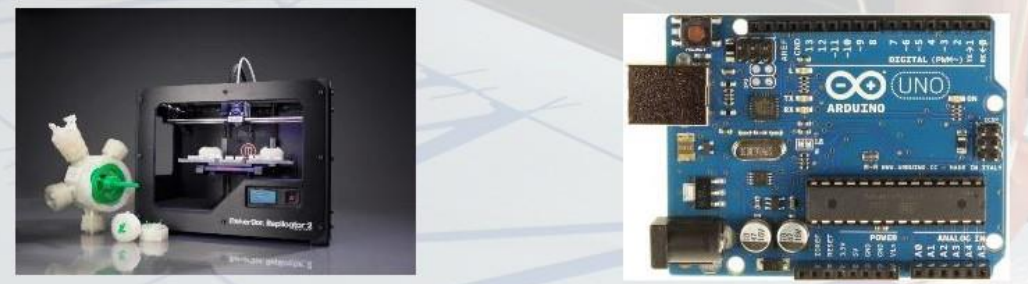

Shared access to non-profit and commercial makerspaces.
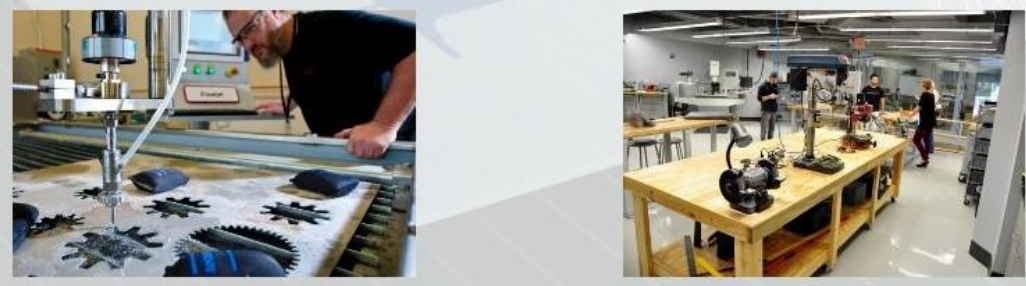


\section{Future Digital Manufacturers}

\section{Inspiration to Innovation to Making}
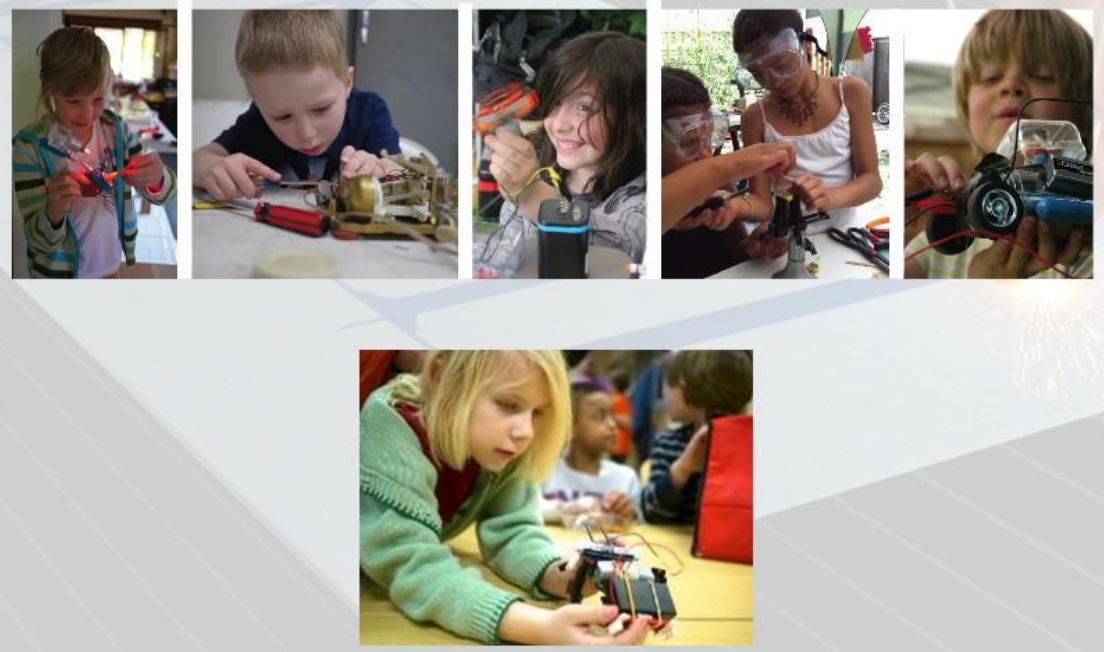

\section{Thank you}

For questions or comments, please contact the Advanced Manufacturing National Program Office amnpo@nist.gov

\section{WWW.manufacturing.gov}

$$
\text { 301-975-2830 }
$$

Unless otherwise labeled, images are courtesy of The White House, the National Institute of Standards and Technology, and Shutterstock 


\section{Presentation 1: An Analysis of Cyber Physical Vulnerabilities in Additive Manufacturing}

\section{Christopher B. Williams}

Associate Professor, Virginia Tech Department of Mechanical Engineering

Key Discussion Points:

- Current research in Cyber Physical Systems is focused on Supervisory Control and Data Acquisition (SCADA) systems, but Additive Manufacturing is different.

- Researchers were able to intercept a job initialization file and decode it, allowing attackers to potentially alter printer parameters mid-print. The STL (or newer AMF) standard files are especially vulnerable to attacks which alter a design.

- The presenters described an experiment run on students at Virginia Tech. Seven groups of students were given an "extra credit" assignment to design a standard dog bone, print it, and test it. An exploit was easily developed which inserted a void in the STL file. Students failed to recognize any anomalies prior to printing and testing. No students correctly diagnosed the anomalies as a cybersecurity problem.

- Recommendations include improved quality control processes, hashing, improved process monitoring, and operator training.

- Some attendees commented that other forms of manufacturing have similar vulnerabilities.

- Cybersecurity solutions should be built under the assumption that manufacturers are not cybersecurity experts. 


\title{
An Analysis of Cyber-Physical Vulnerabilities in Additive Manufacturing
}

\author{
Logan Sturm ${ }^{1}$, Christopher B. Williams ${ }^{1}$, Jaime A. Camelio ${ }^{2}$, Jules White ${ }^{3}$, Robert Parker ${ }^{4}$ \\ ${ }^{1}$ Department of Mechanical Engineering, ${ }^{2}$ Department of Industrial \& Systems Engineering, ${ }^{3}$ Department \\ of Computer Science \\ ${ }^{1,2}$ Virginia Tech, ${ }^{3}$ Vanderbilt University, ${ }^{4}$ VT-ARC \\ ${ }^{1,2,4}$ Blacksburg, VA, USA
}

\section{Keywords-Additive Manufacturing; 3D Printing; Cyber/Physical security}

\section{EXTENDED ABSTRACT}

While the "digital thread" of advanced manufacturing technologies enables a more efficient design process, it also presents opportunities for cyber-attacks to impact the physical word. A cyber-attack on manufacturing systems could cause injury to plant workers and damage to the machine itself. More insidiously, an attack could be designed to cause a process to produce faulty parts that might find their way into end-user products. With the rise in both the number of cyber-physical systems connected to networks and in malicious cyber-attacks, there is a clear need for research to understand the vulnerabilities of cyber-physical systems. While methods exist for detecting cyber-attacks on computer systems, no such research has been done on detecting an attack from the physical parts created by the attack.

In this work, the authors scope their research solely on Additive Manufacturing (AM; also referred to as "3D Printing") technologies. The AM process chain has unique vulnerabilities that warrant a detailed investigation due to their ability to fabricate parts in a layer-wise fashion. Because of the potential damage from a cyber-physical attack, there is a need to look at AM systems to determine what vulnerabilities exist and how to prevent and mitigate the threat of cyber-attacks.

The digital nature of the AM process chain provides an opportunity for a cyber-attack to cross into the physical world. There are four main steps on the process chain where an attack could take place: the CAD model, the .STL file, the toolpath file, and the physical machine itself. While the authors will discuss attack vectors at each of these steps within the process chain, their focus will be on vulnerabilities within the .STL file as it is the one vulnerability that does not require specific modification for an individual AM machine. As STL file creation occurs at the beginning of the process chain and the file format is standardized across every AM machine, a focused attack could have severe implications across an AM production line regardless of the machine type or manufacturer.

The current defacto standard in AM, the STL file only contains the surface information of the part. This information is stored as a list of triangular elements (specified by the a set of $\mathrm{x}, \mathrm{y}$, and $\mathrm{z}$ coordinates of three vertices) in ASCII or binary format. An attack that simply edits the STL file could subtly alter the part geometry. STL file edits/attacks could take the form of (i) part scaling, (ii) surface indents or protrusions, (iii) vertex movement, and (iv) insertion of internal voids within the part. While most of these vectors affect the surface of the part geometry - and thus could possibly detected using standard quality control dimensional measurements - the void attack is completely enclosed inside the model. Because of this, such an attack would be undetectable by dimensional measurements and may be difficult or impossible to find visually. The use of supporting material in many processes also renders the void undetectable by weighing, since the void is filled with a structurally deficient, but equivalently dense material.

To ascertain the potential impact of this specific attack, two experiments were performed. First the authors evaluated the effect of a "printed void" on the mechanical strength of a printed specimen. Several ASTM Standard D638-10 tensile test specimens with and without voids were printed on via Powder Bed Fusion (a Sinterstation 
2500 Plus machine) using Nylon 12 powder. Upon testing, all of the specimens containing voids fractured at the void location, while the specimens without voids failed normally. The average reduction in yield load was $14 \%$, from $1085 \mathrm{~N}$ to $930 \mathrm{~N}$, and the strain at failure was reduced from $10.4 \%$ to $5.8 \%$.

Second, a case study was performed to determine the feasibility of a cyber-attack on a simple AM system and to evaluate the ability of AM operators to detect an attack. In this experiment, upper-level and graduate engineering students were challenged to manufacture and test a tensile test specimen. Unknown to the participants, the computer used was infected with .STL attack software that automatically inserted voids into their files before fabrication. Upon completion of the printing, none of the participants detected the presence of the voids in their parts. Upon breaking the part, all participant teams identified that their parts failed prematurely. Two teams detected the presence of a void at the fracture location; however both of these teams concluded that the placement was due to problems with the machine. Two teams did not notice the voids and attributed the failure to the anisotropic nature of additively manufactured parts.

Based on the results of this study, it appears that a real threat from cyber-physical attacks exists and that further research needs to be done on how to mitigate such attacks. The inclusion of software checks, hashing, process monitoring, and worker training are proposed as methods of reducing these threats. Future work includes the development of physical hashing techniques and of improved side channel process monitoring and control. 
Presentation:

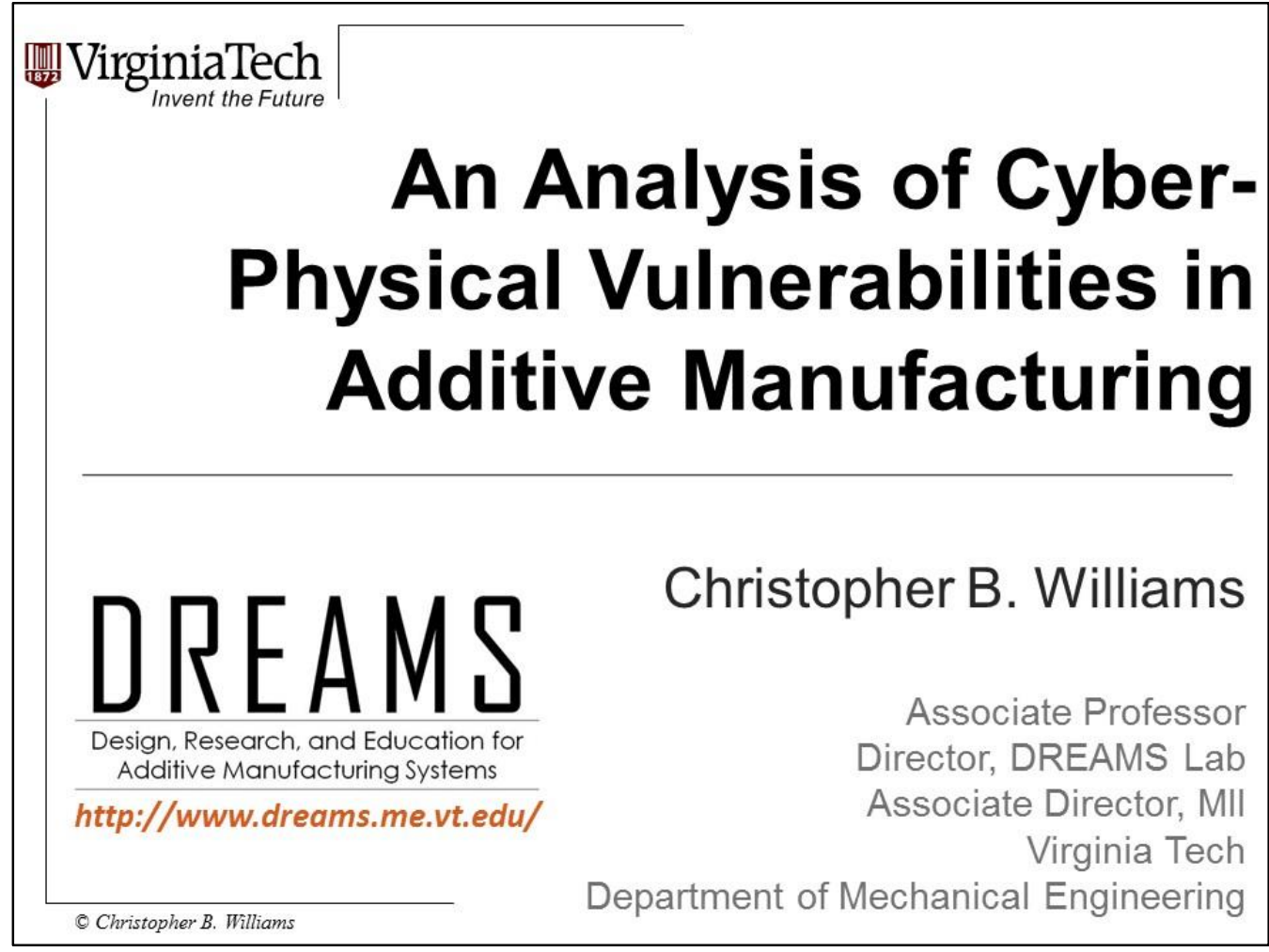

\section{VirginiaTech Invent the Future}

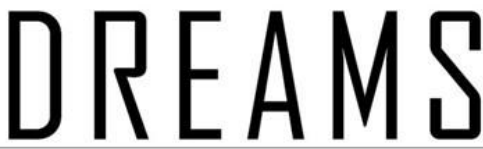

Design, Research, and Education for Additive Manufacturing Systems

http://www.me.vt.edu/dreams

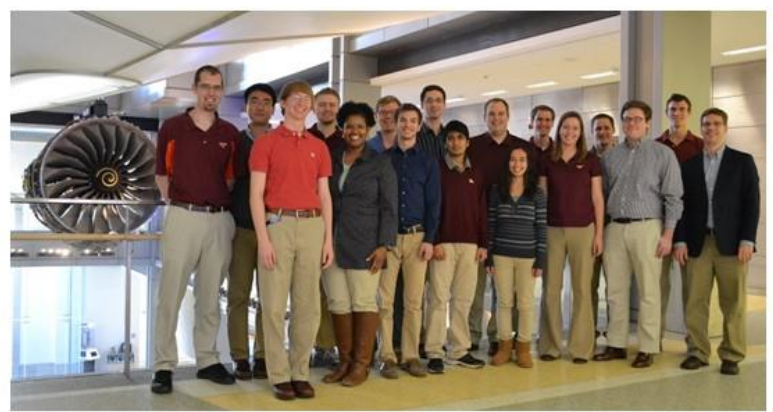

\section{Vision:}

To advance the science, state of the art, and pedagogy of AM, and thereby lead the transition of today's layered fabrication techniques into viable platforms for the realization of end-use products. 

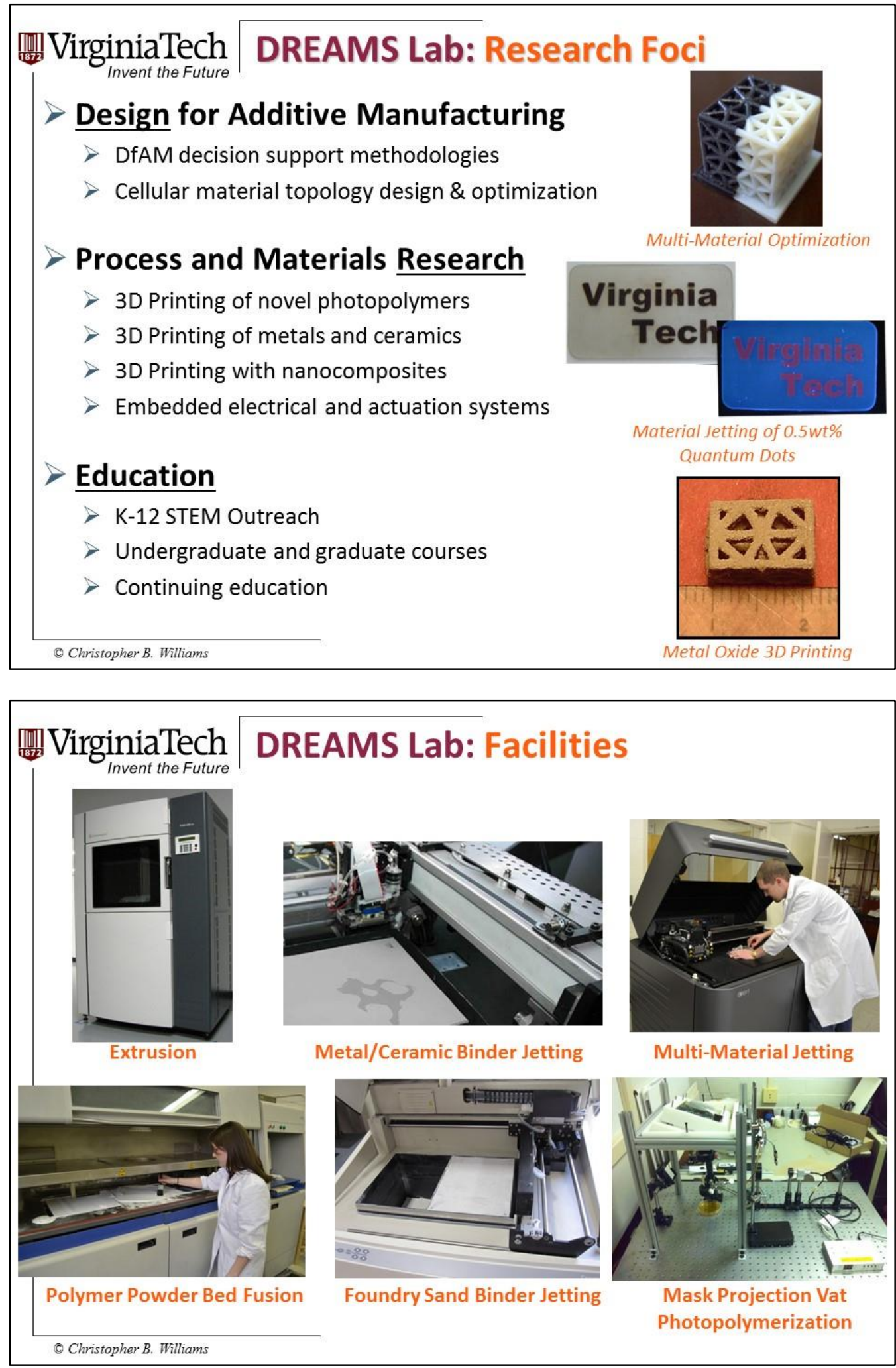


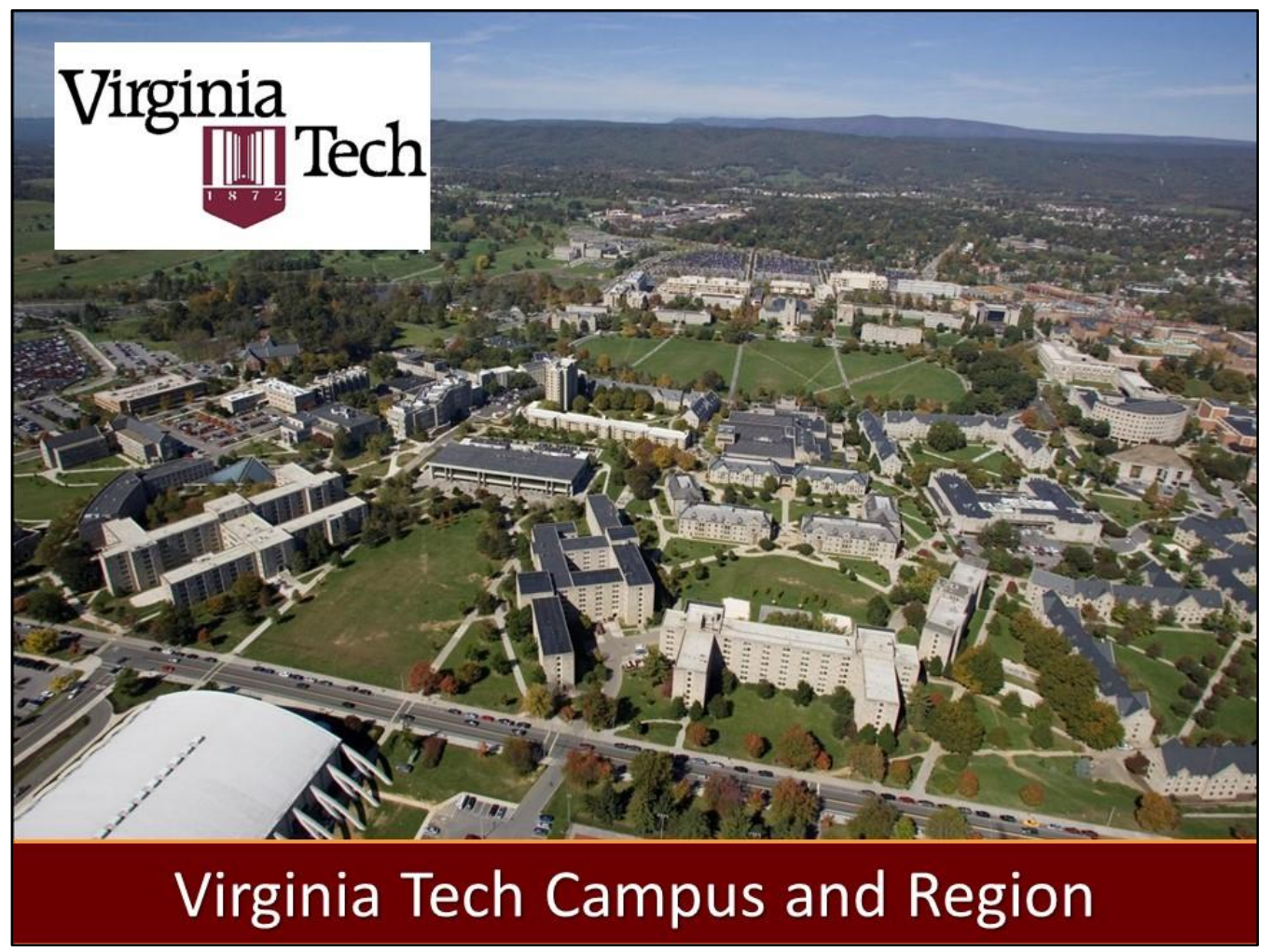

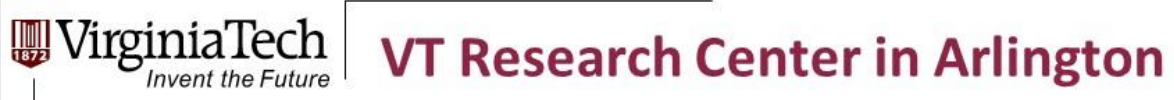

The Virginia Tech Research Center in Arlington (VTRCA) opened in June 2011 and is $144,000 \mathrm{GSF}$.

The College of Engineering has 19,000 GSF and ICTAS is occupies an additional 7,000 GSF.
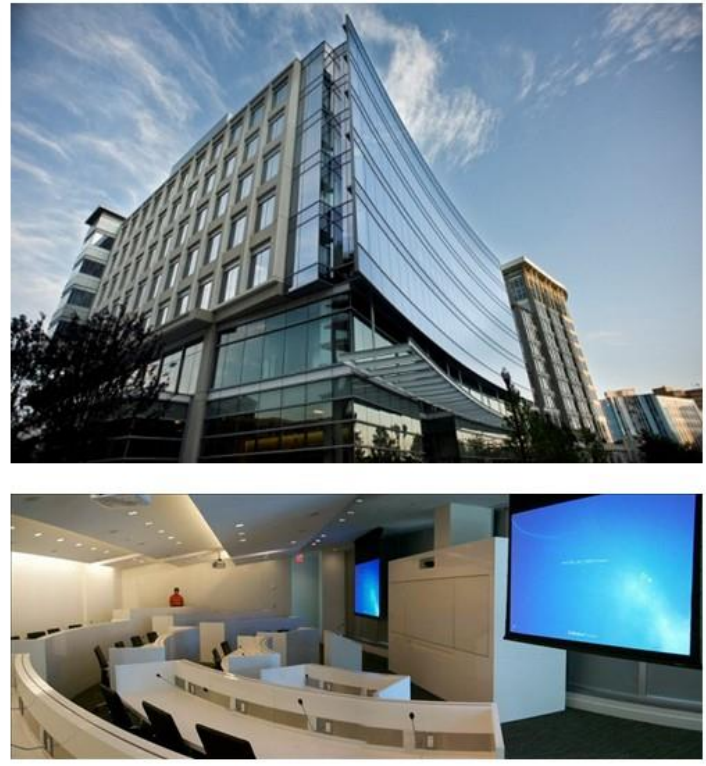


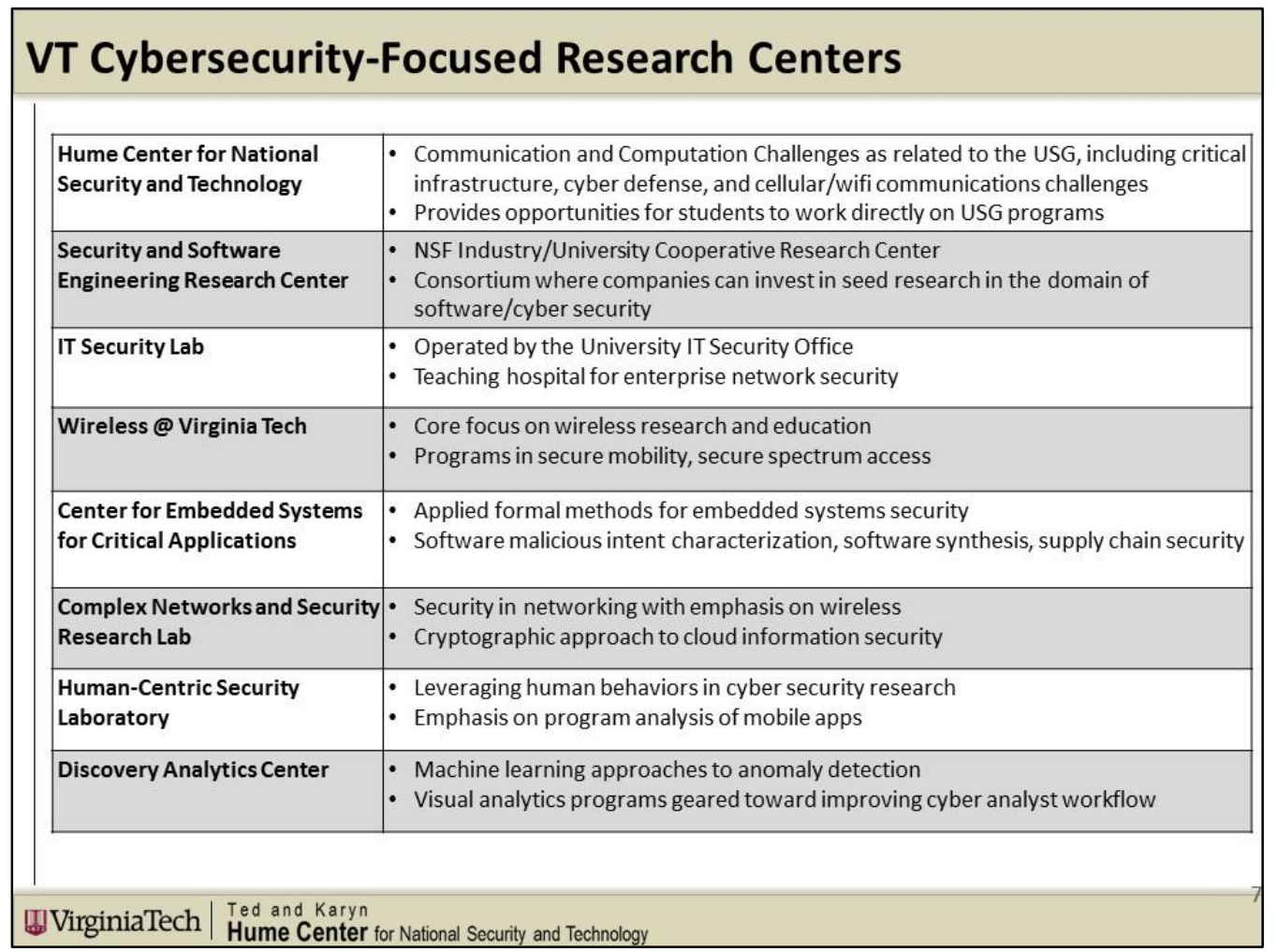

\section{VT Cybersecurity-Focused Research Centers}

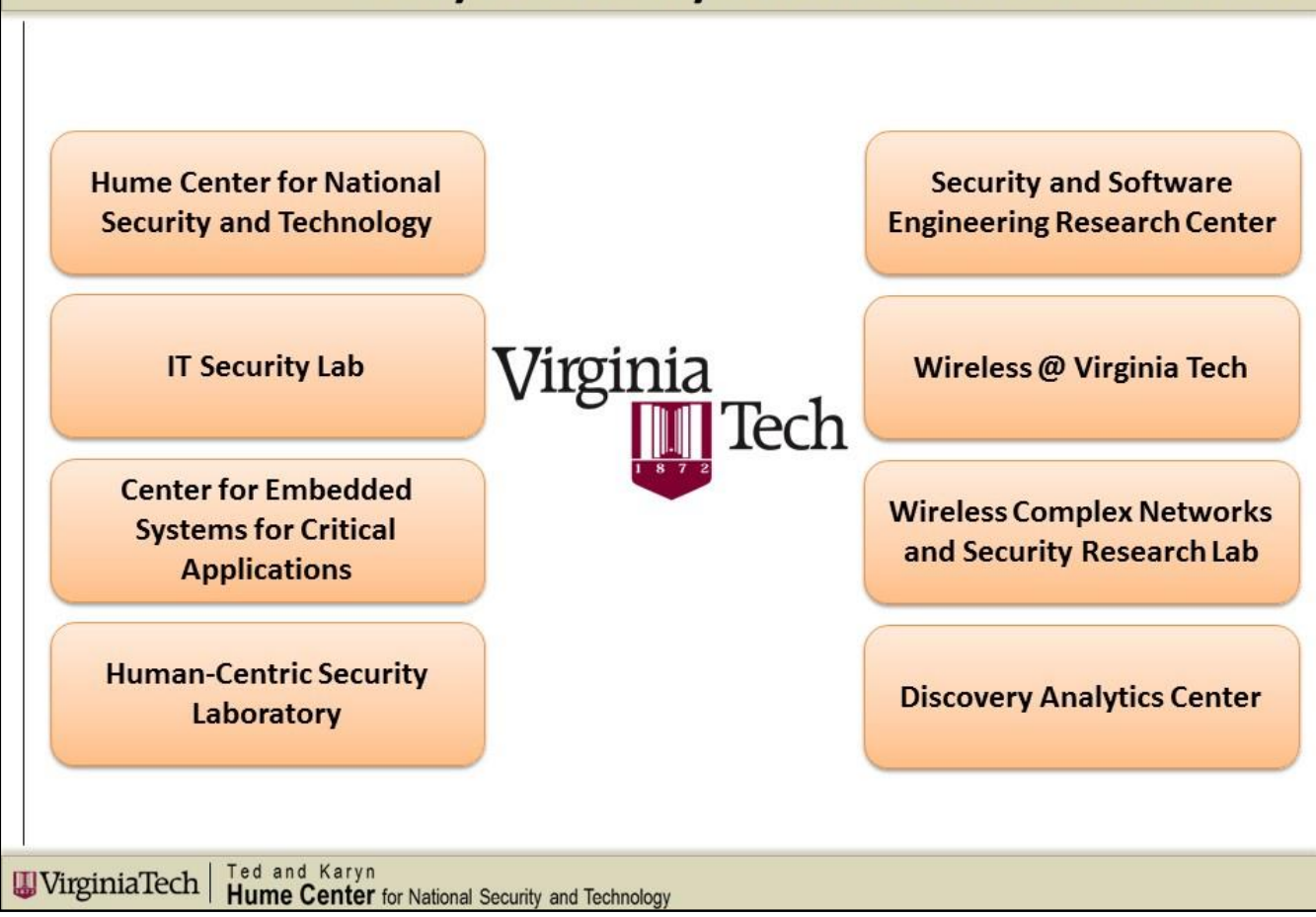




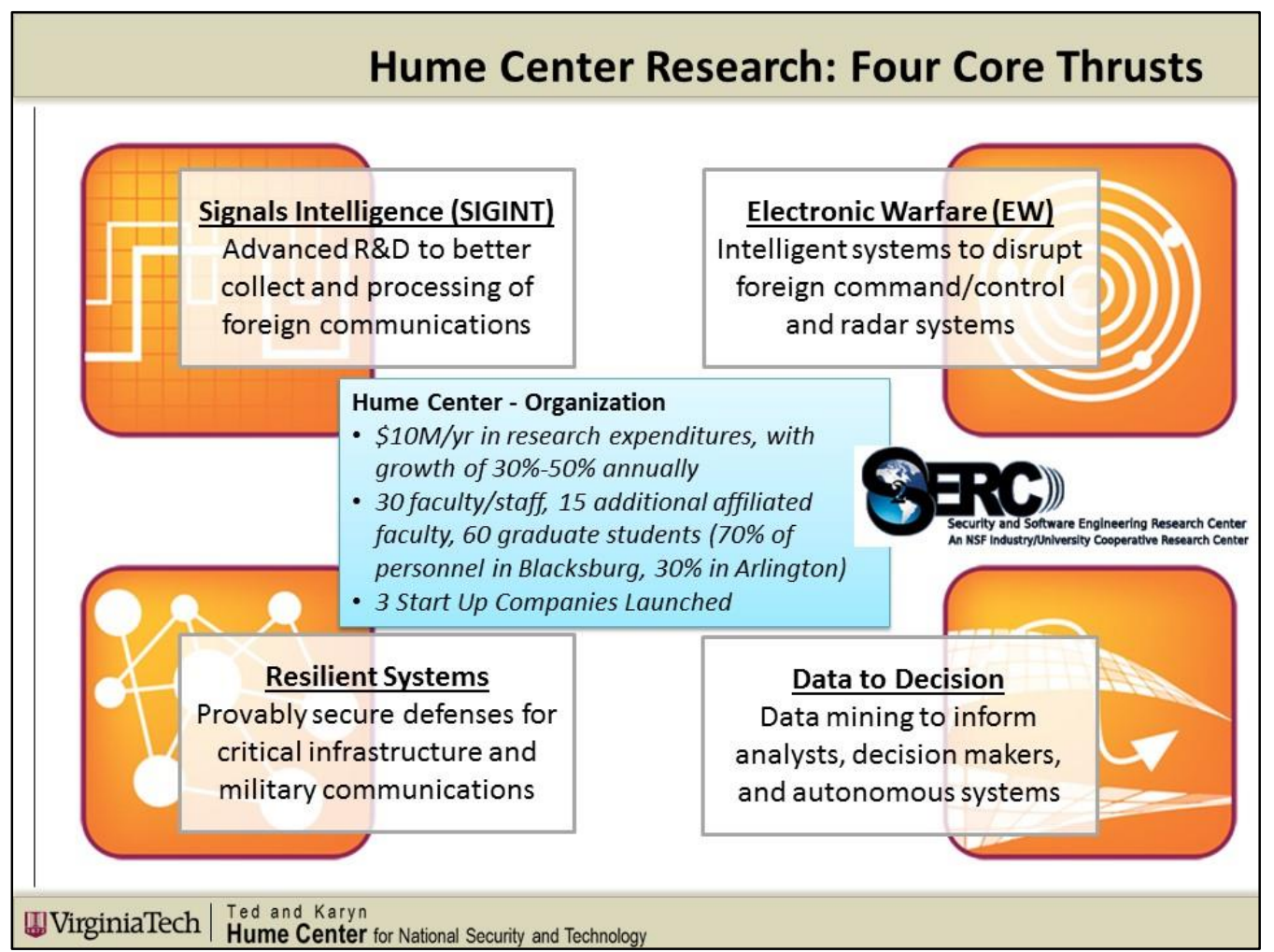

Mobile Security \& Privacy
Smartphone Security
Cellular Infrastructure Security
$4 \mathrm{G} / 5 \mathrm{G}$ Security




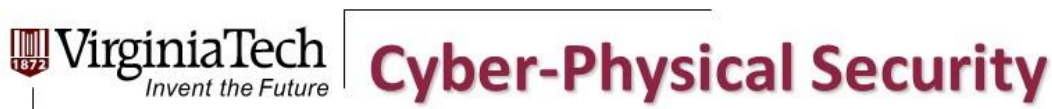

\section{Cyber-Physical Systems}

$>$ "a system of computational elements controlling physical entities"

\section{Cyber-Physical Attacks}

Malware attacks on PCs that affect physical systems

\section{Examples:}

Electrical power generation and management (Smart Grid)

$>$ Water and waste management

$>$ "Hacking people" (insulin pumps \& pacemakers)

$>$ Stuxnet (Iranian nuclear centrifuges)

\section{Current Research Focus}

Supervisory Control and Data Acquisition (SCADA) Systems

\section{WirginiaTech Cyber-Physical Security}

\section{$>$ Opportunity}

$>$ "Research in computer security has focused traditionally on the protection of information. Researchers have not considered how attacks affect the physical world." [Cardenas et al, 2009]

\section{$>$ Current Research Focus}

$>$ Supervisory Control and Data Acquisition (SCADA) Systems

\section{However, is this enough to protect our Manufacturing Infrastructure?}




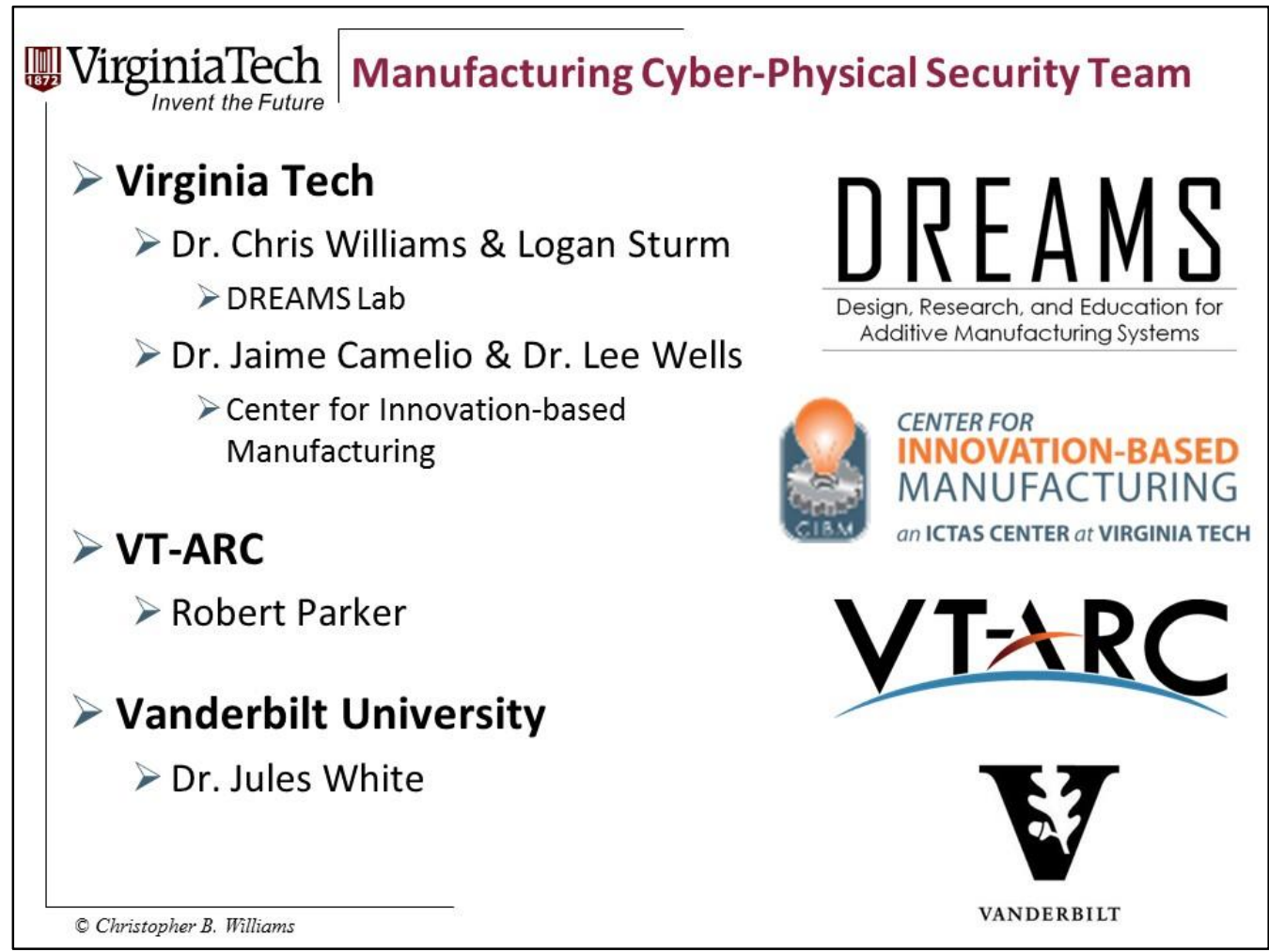

\section{Manufacturing Cyber-Physical Vulnerability Mapping}

- Generic Vulnerability Visualization Tool (VVT)

- Vulnerabilities - Any potential loss of design intent

- Intentional or unintentional

- Tracking thread progression through a system

- Digital thread

- Physical thread

- Cyber-Physical thread

- Human thread

- Vulnerabilities manifestation

- Inter/Intra section of thread entities

- Thread entity enters/leaves

- Identified vulnerabilities assessed by a team of experts for attack surface analyses

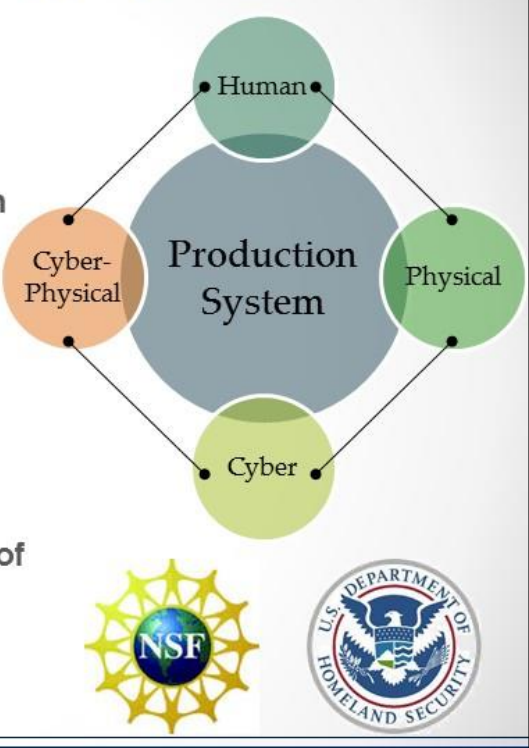



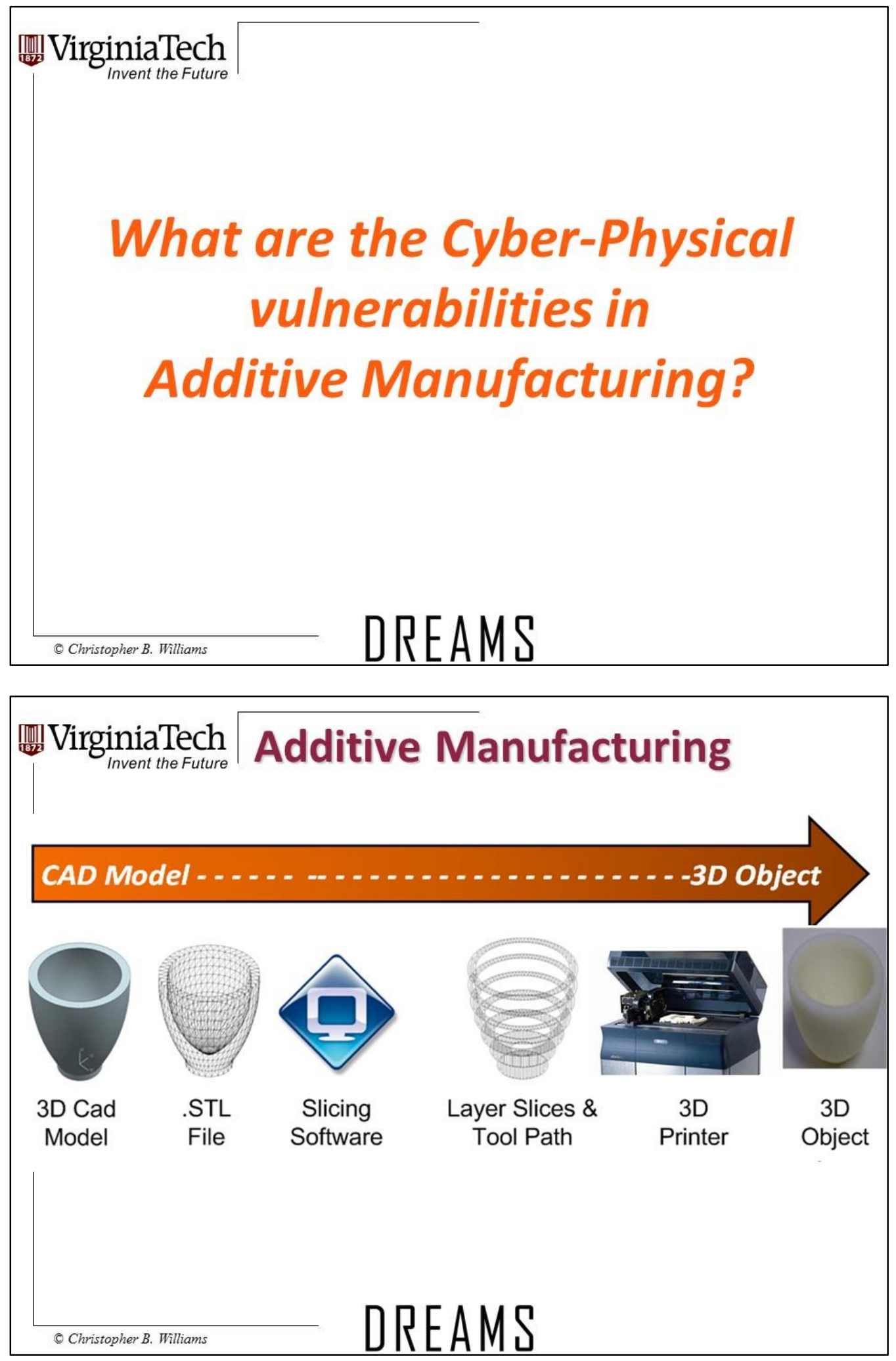

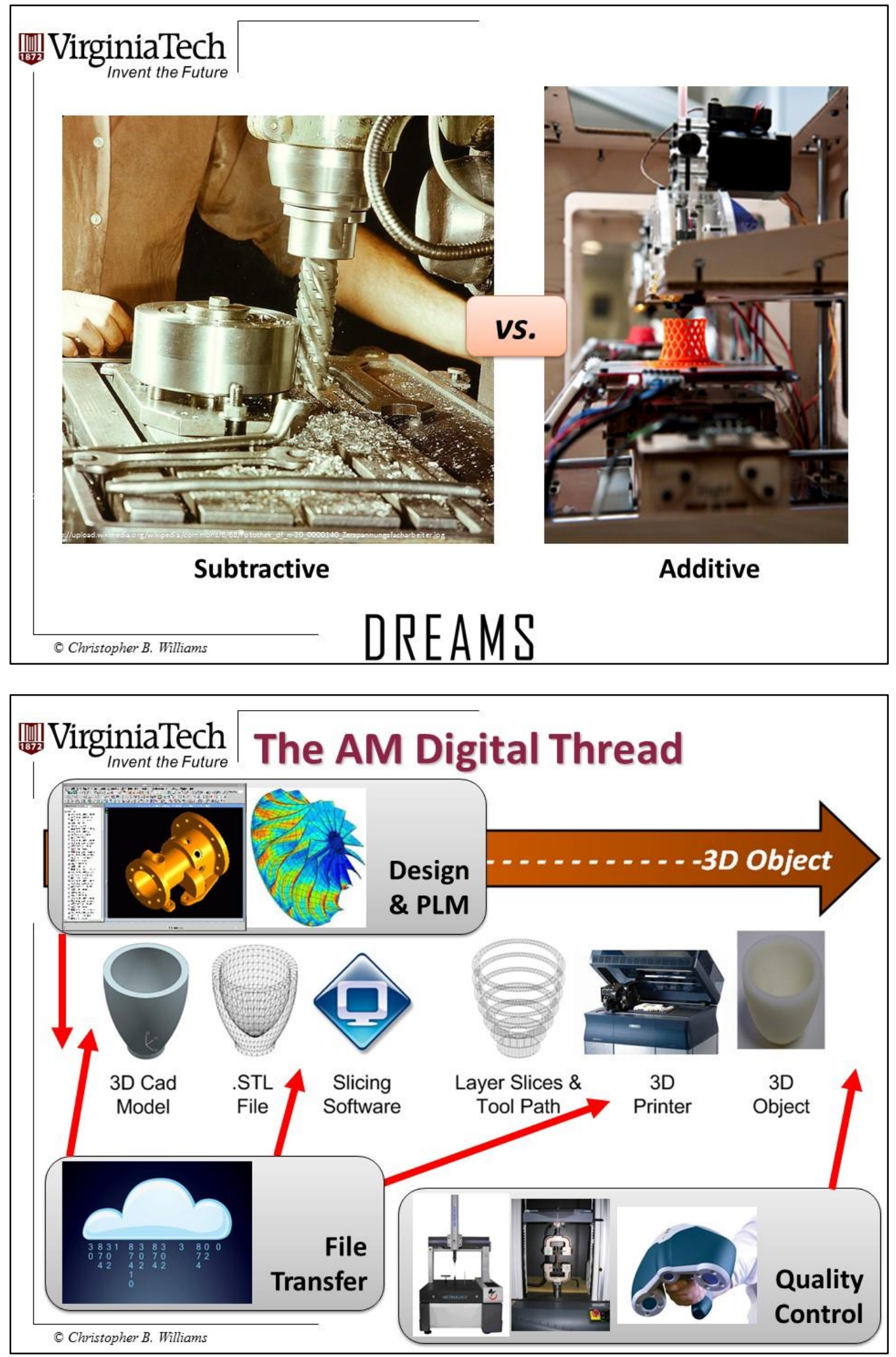


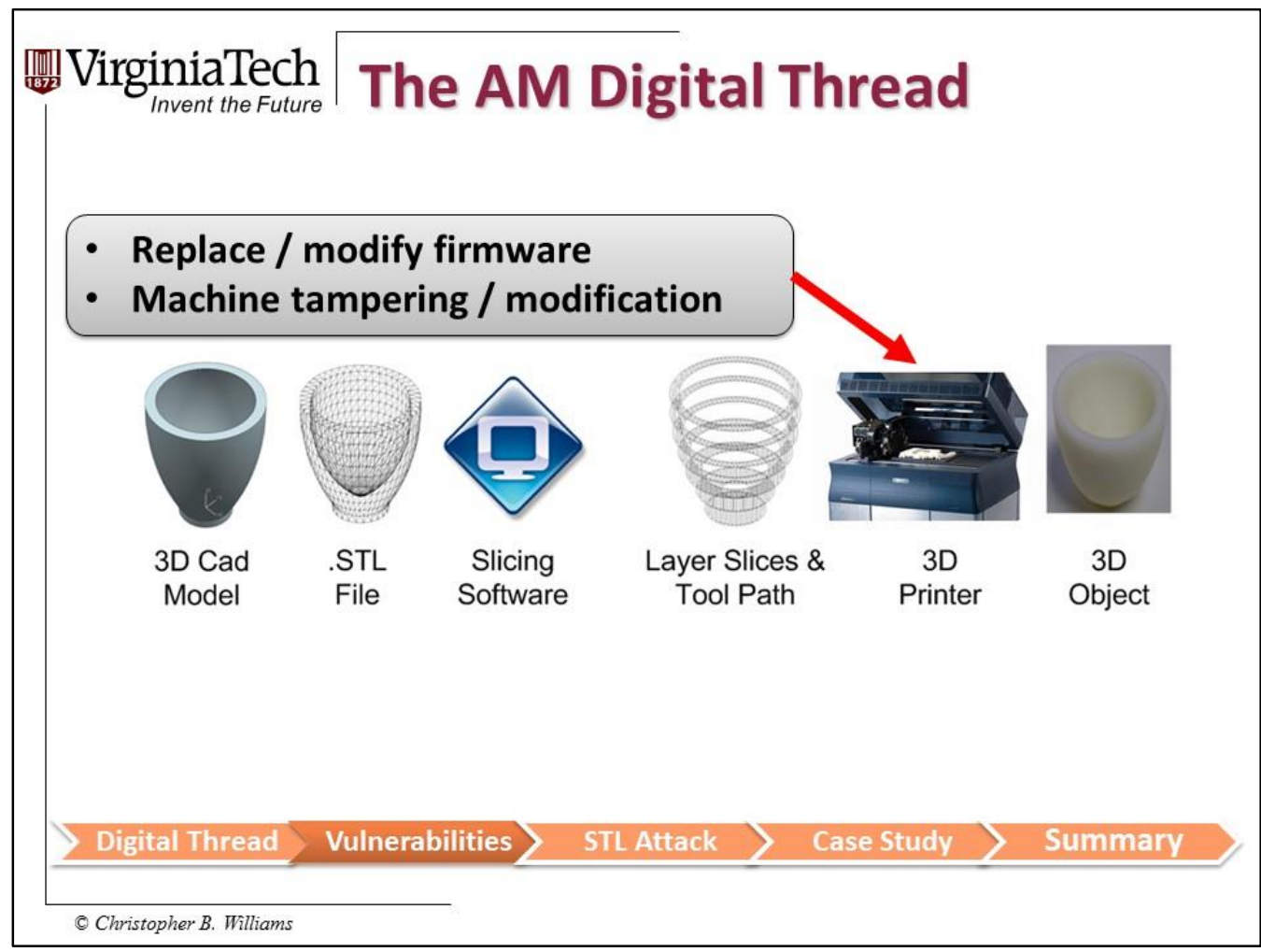

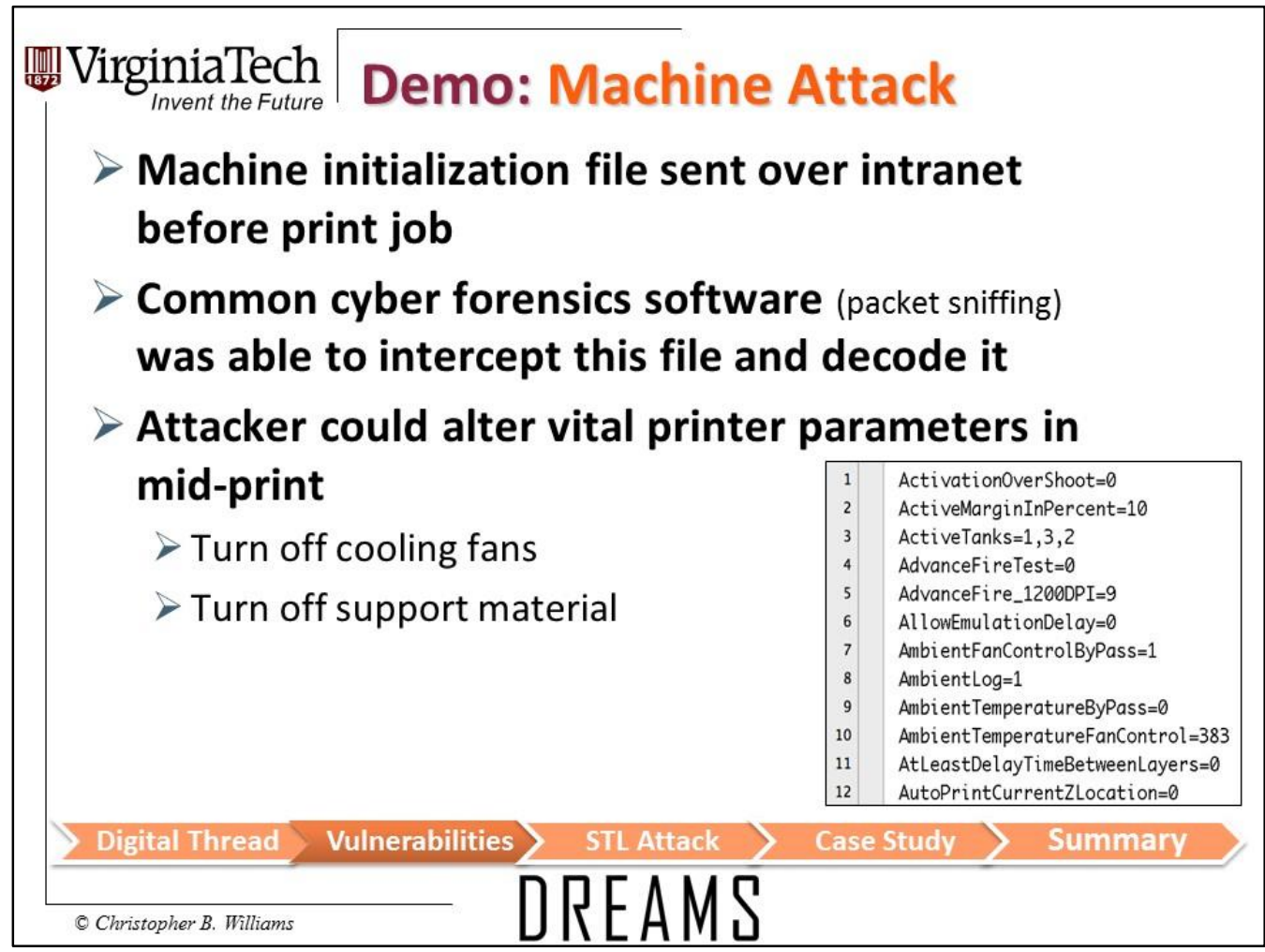




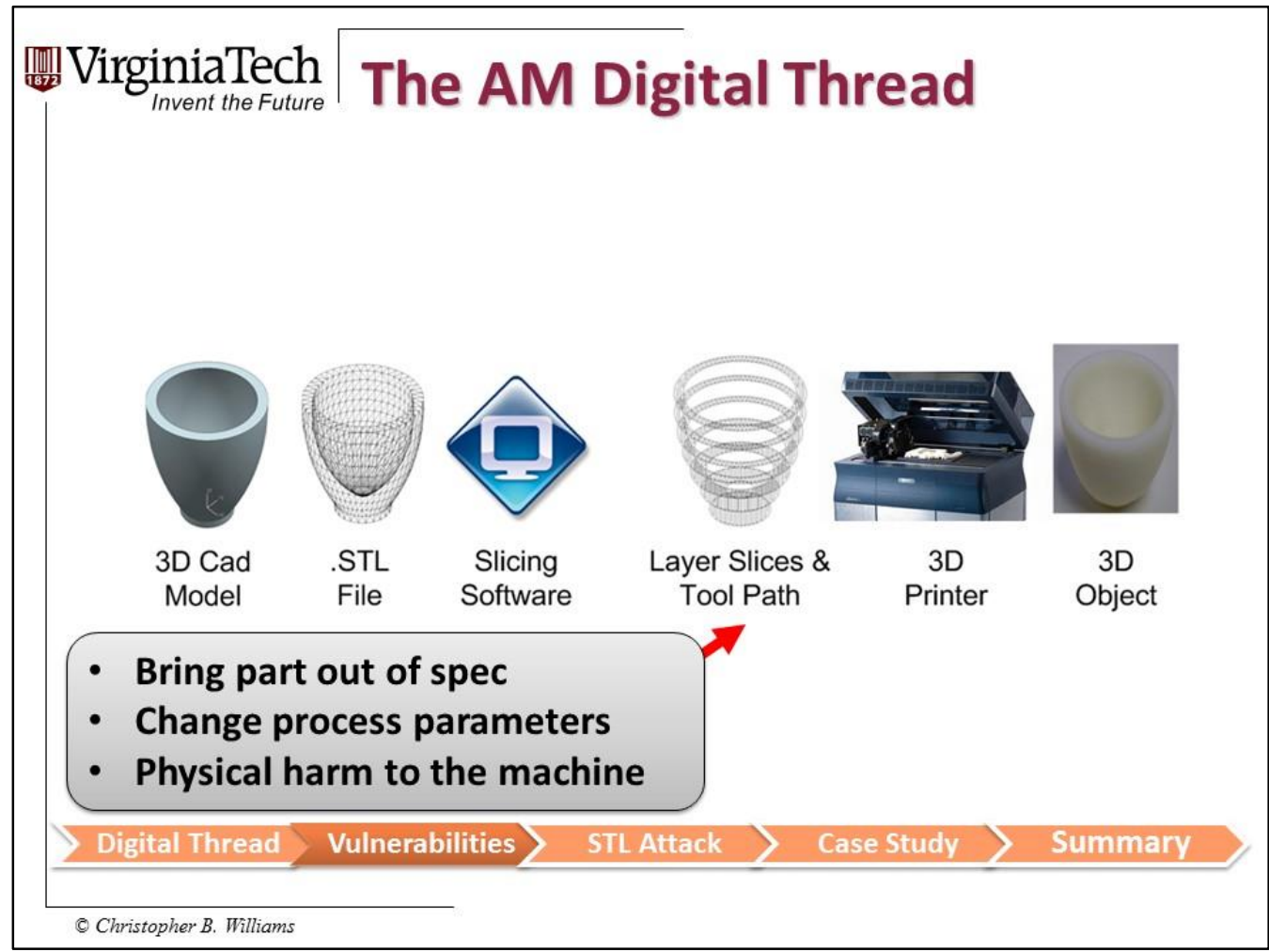

\section{DirginiaTech The AM Digital Thread}

- File interception / augmentation

- Bring part out of specification

- Add unwanted features

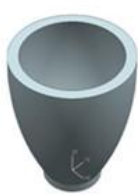

3D Cad Model

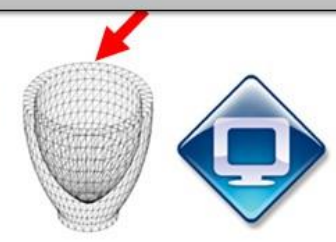

STL

File
Slicing
Software

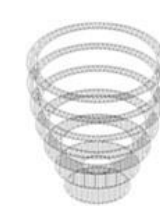

Layer Slices \& Tool Path

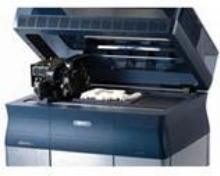

3D

Printer

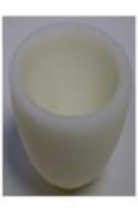

3D

Object

\section{Digital Thread $>$ Vulnerabilities $>$ STL Attack $>$ Case Study $>$ Summary}




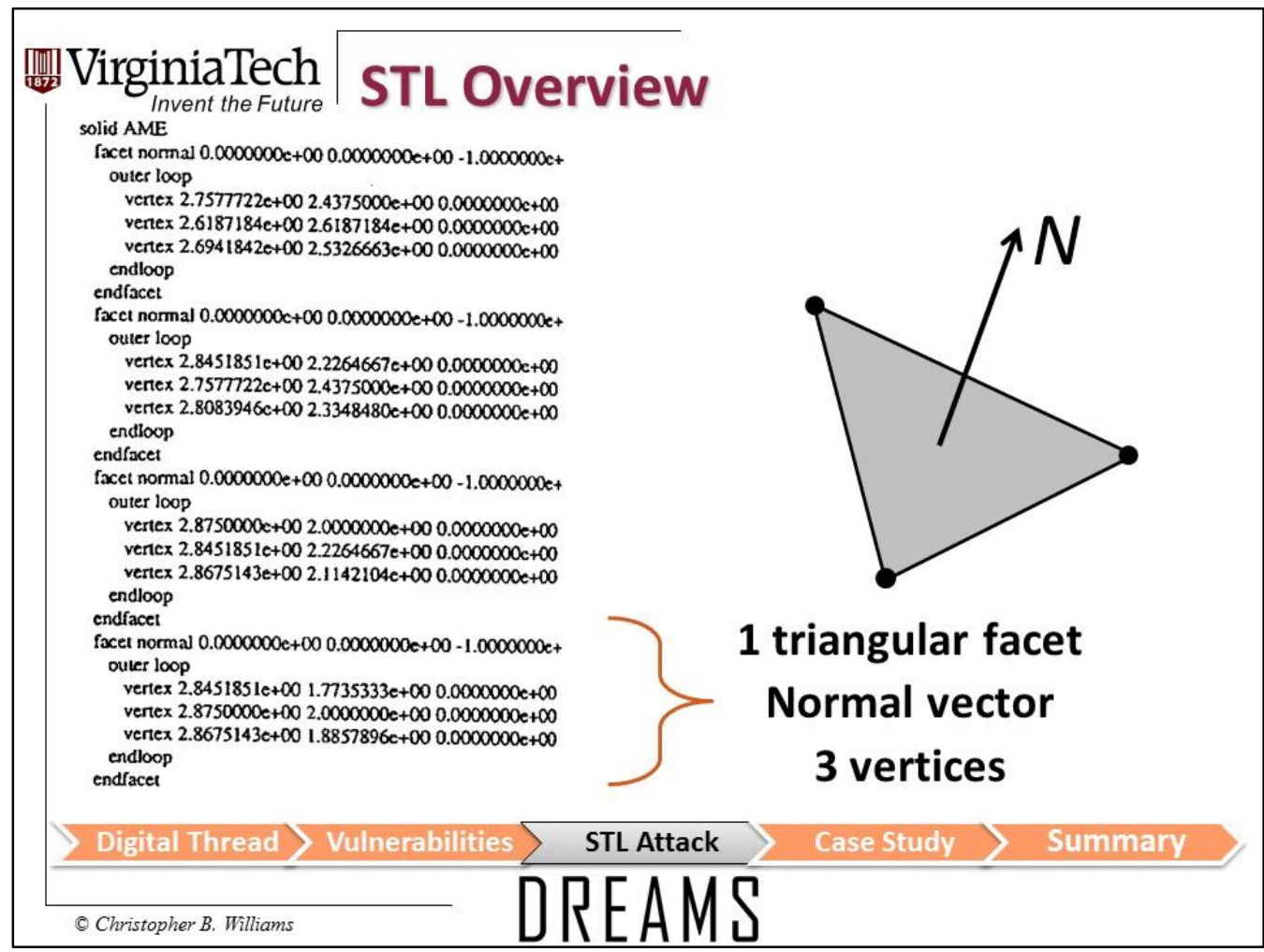

\section{顓VirginiaTech \\ Increase/decrease part scale \\ Part can be brought out of specification \\ Surface dimples / protrusions \\ Bumps or voids added to the part surface}

STL Attack: Exterior Attacks

\section{Moved vertices}

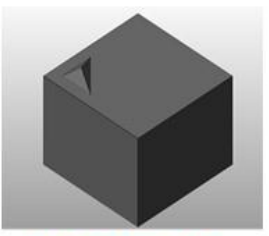

Add protrusion

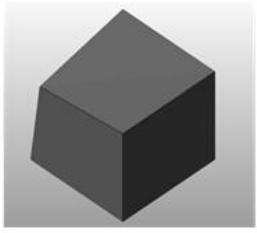

Move vertex inward

Part features can be altered by moving vertices 

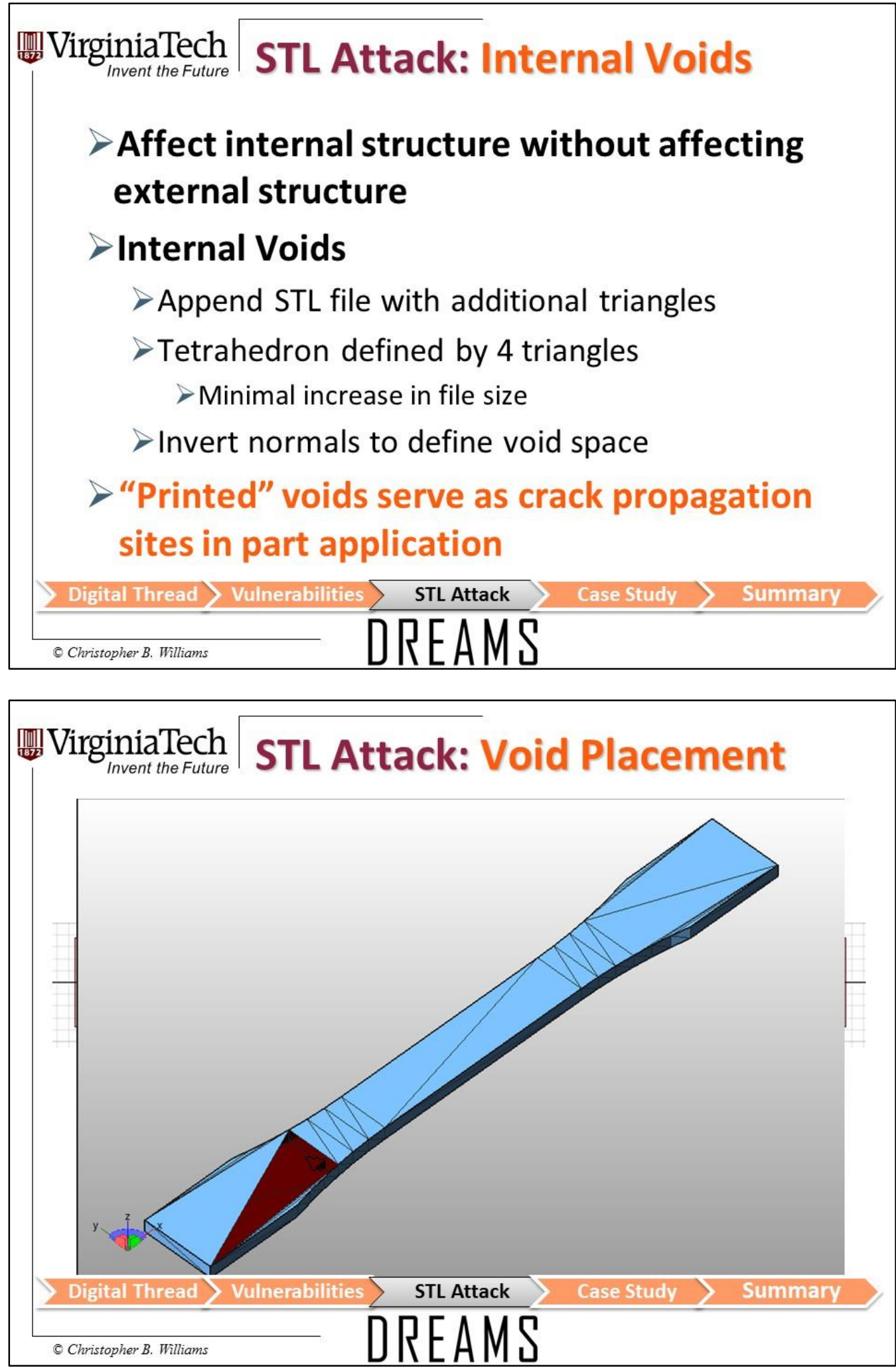


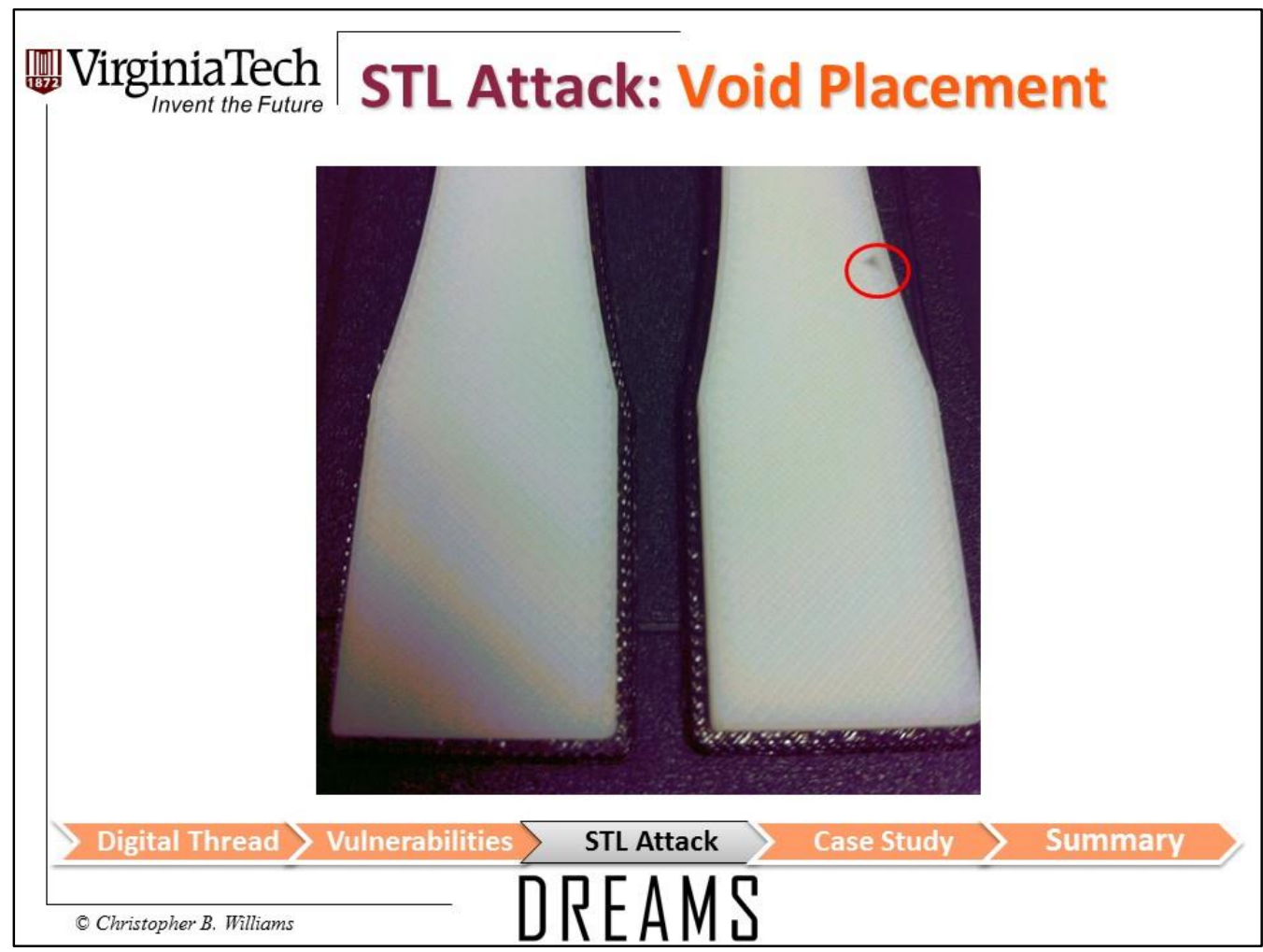

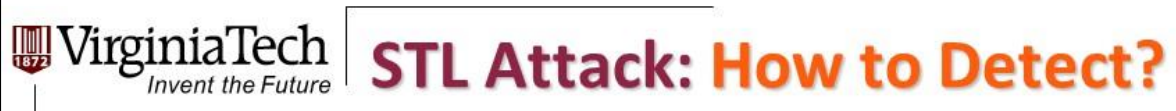

$>$ External surface is untouched

Mass difference is undetectable

$>$ In-situ monitoring is validated against STL file

$>$ Human visual inspection of every layer?

$>$ CT scan of each completed part? 


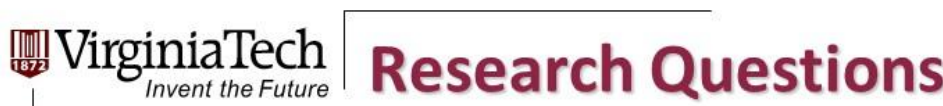

$>$ Can one execute a void attack with the information provided in a STL file?

\section{How do inserted voids affect part strength?}

$>$ Is the void placed in a structurally important area of the part?

$>$ Will the void size/shape cause a failure?

\section{Can the attack be detected?}

$>$ Does the attack cause a noticeable delay?

$>$ Does the attack set off any alerts in software checks?

$>$ Is there a noticeable change in file size?

\section{Digital Thread $>$ Vulnerabilities $>$ STL Attack $>$ Case Study $>$ Summary \\ C Christopher B. Williams \\ DREAMS}

IIIrginiaTech

\section{Why simulate an attack?}

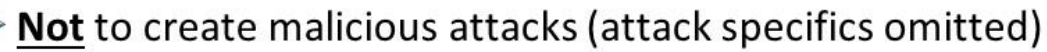

$>$ To gain a better understanding of existing vulnerabilities

To determine if these vulnerabilities are significant

To understand the circumstances that allow attacks to occur

To develop better methods for preventing cyber-physical attacks from occurring

STL file attack chosen for study due to its universality and ease of editing 

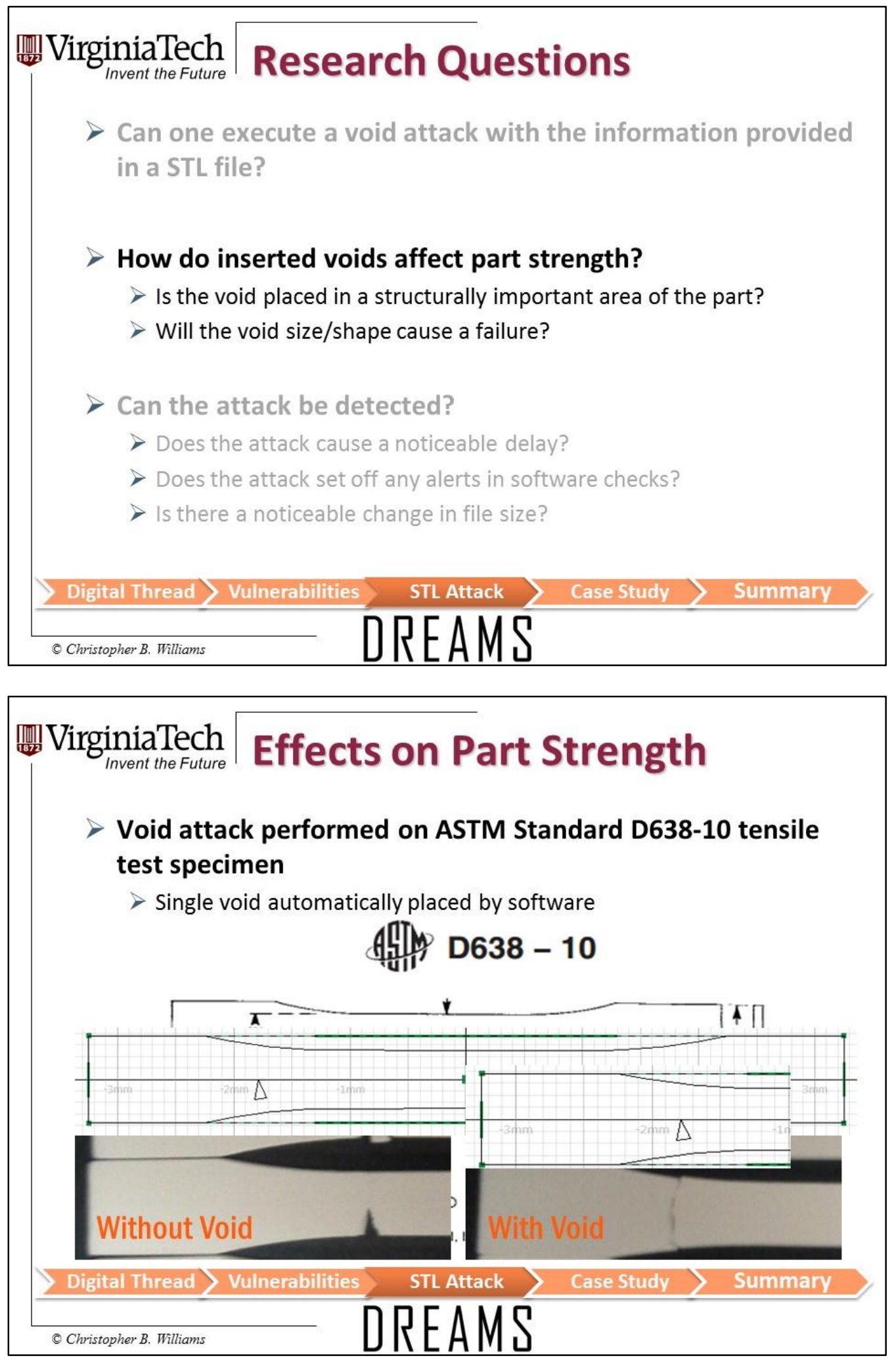

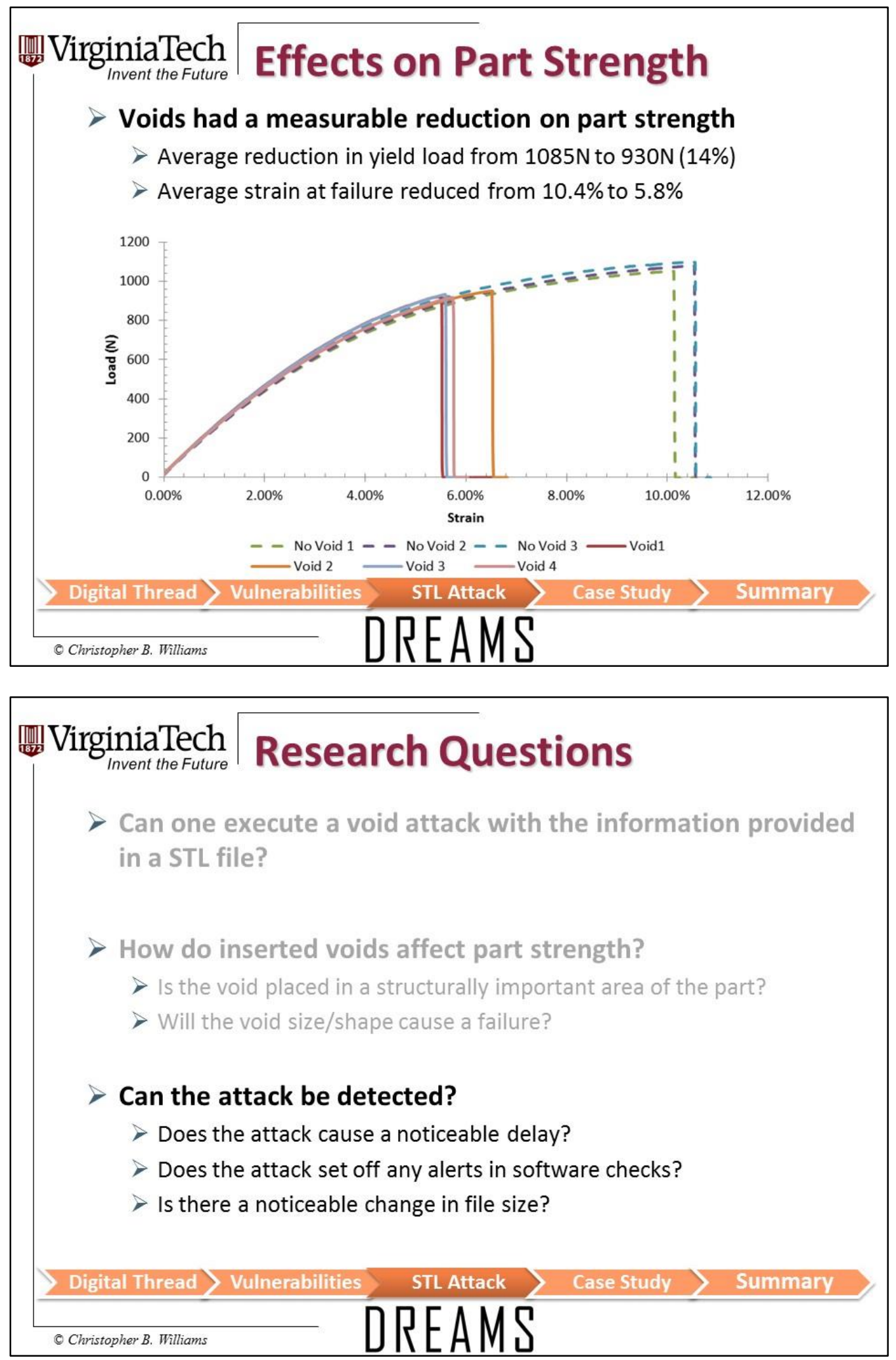

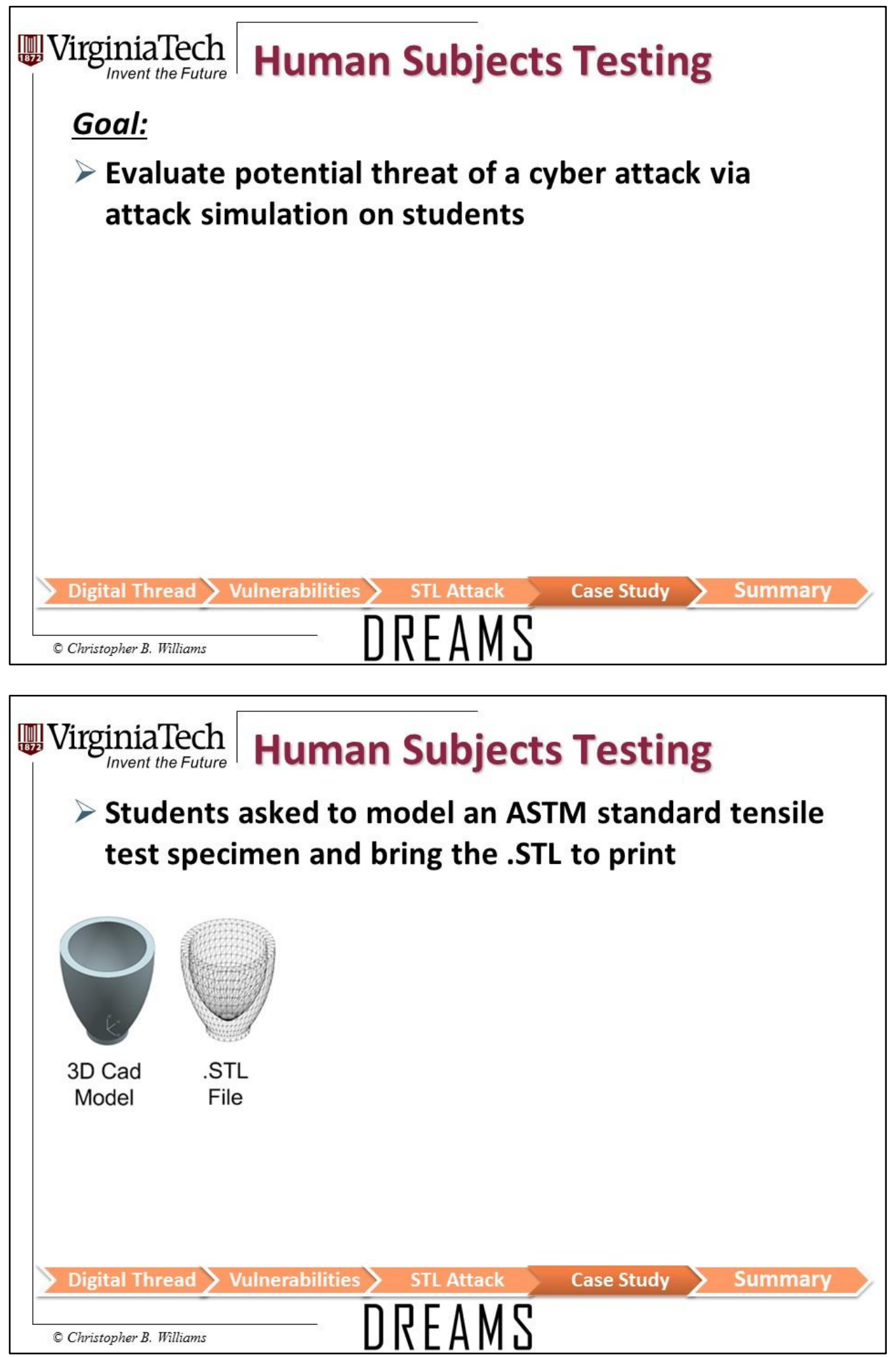


\section{VirginiaTech Human Subjects Testing}

Upon copying the file to the workstation computer the part was attacked (i.e., a void was added)
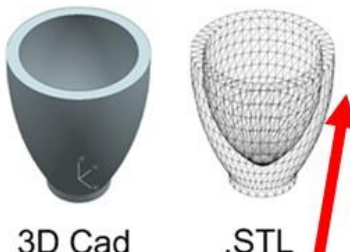

3D Cad Model

File

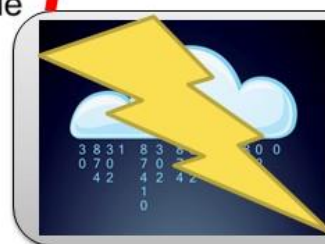

Attack

Occurs

\section{DREAMS}

IniginiaTech Human Subjects Testing

$>$ Students are required to check the part in Netfabb to ensure that it is correct before printing

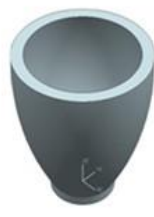

3D Cad Model

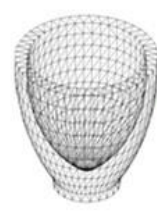

STL

File

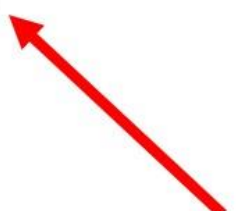




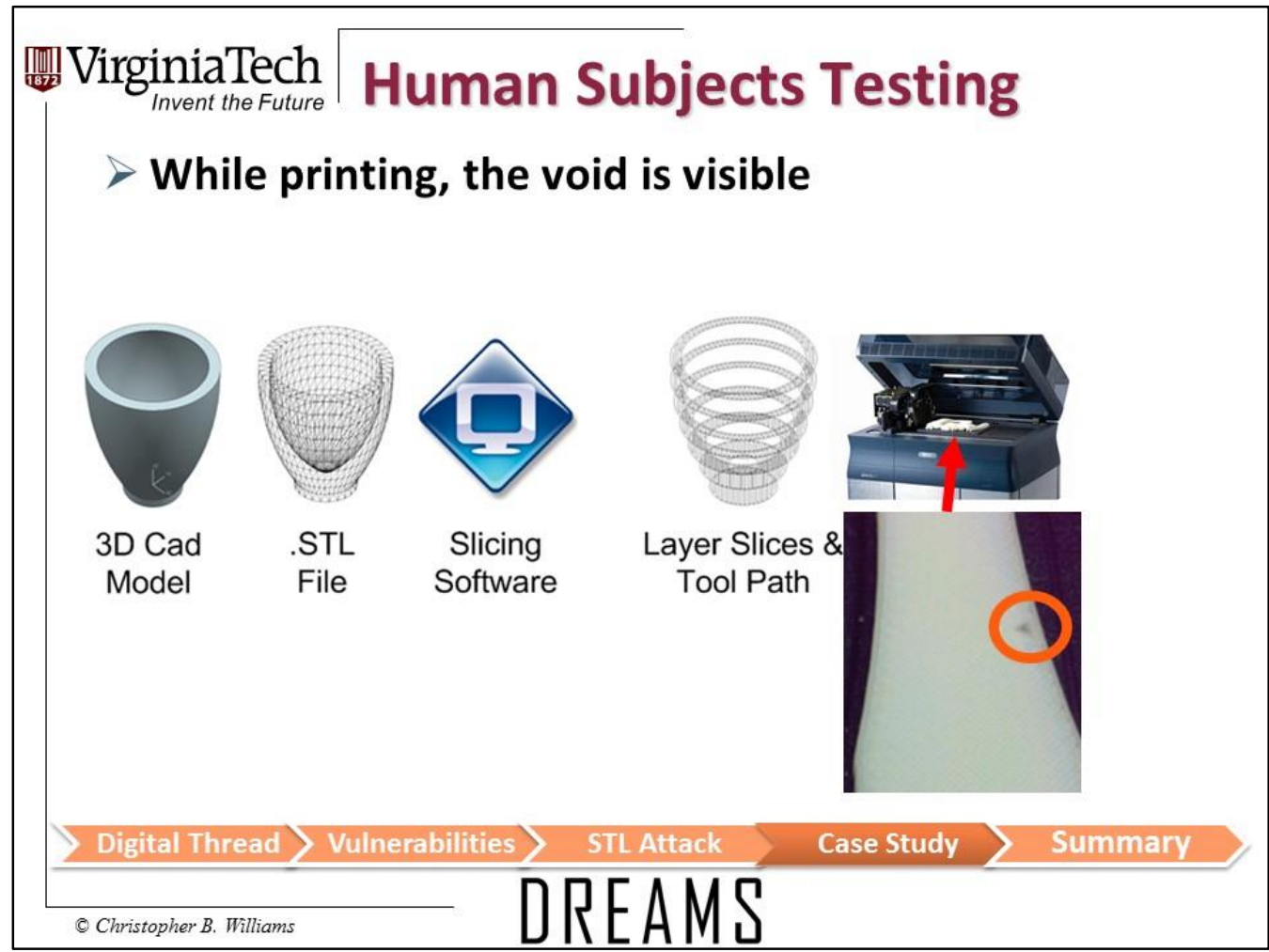

DirginiaTech

After printing, void is not visible

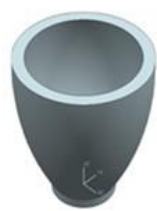

3D Cad Model

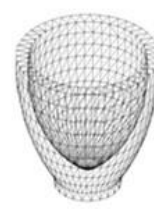

STL File

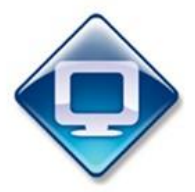

Slicing Software

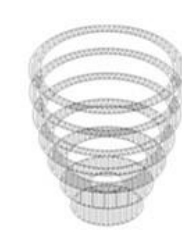
Layer Slices \&
Tool Path

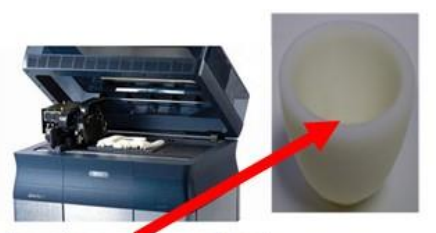

3D Object

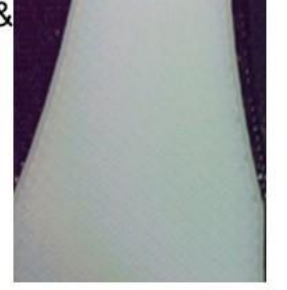


DIVIrginiaTech

$>$ Final part was measured and inspected before being broken on a tensile test machine

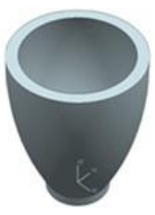

3D Cad Model

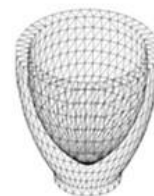

STL

File

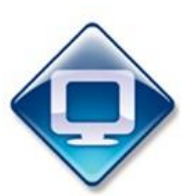

Slicing Software

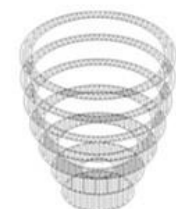

Layer Slices \& Tool Path

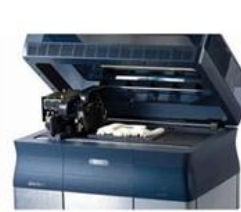

3D Printer

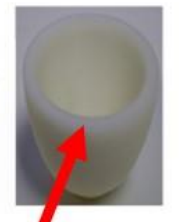

3D Object

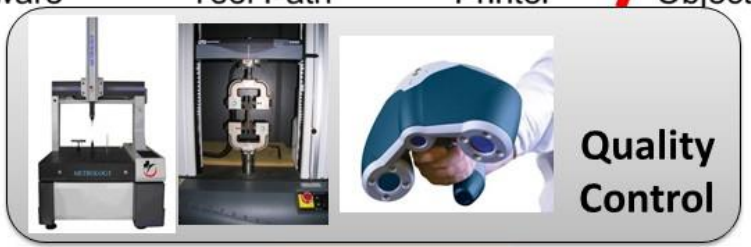

Digital Thread > Vulnerabilities > STL Attack

Case Study

Summary

C Christopher B. Williams

\section{DREAMS}

罗 VirginiaTech Human Subjects Testing

4 groups participated (18 students)

None of the groups detected the void before printing. (Netfabb check)

$>\mathbf{2}$ groups stayed while the parts were built

$>1$ group noticed signs of the voids during the build

$>$ None of the groups indicated seeing the voids after the part was printed (before testing)

All groups identified the part failed prematurely 


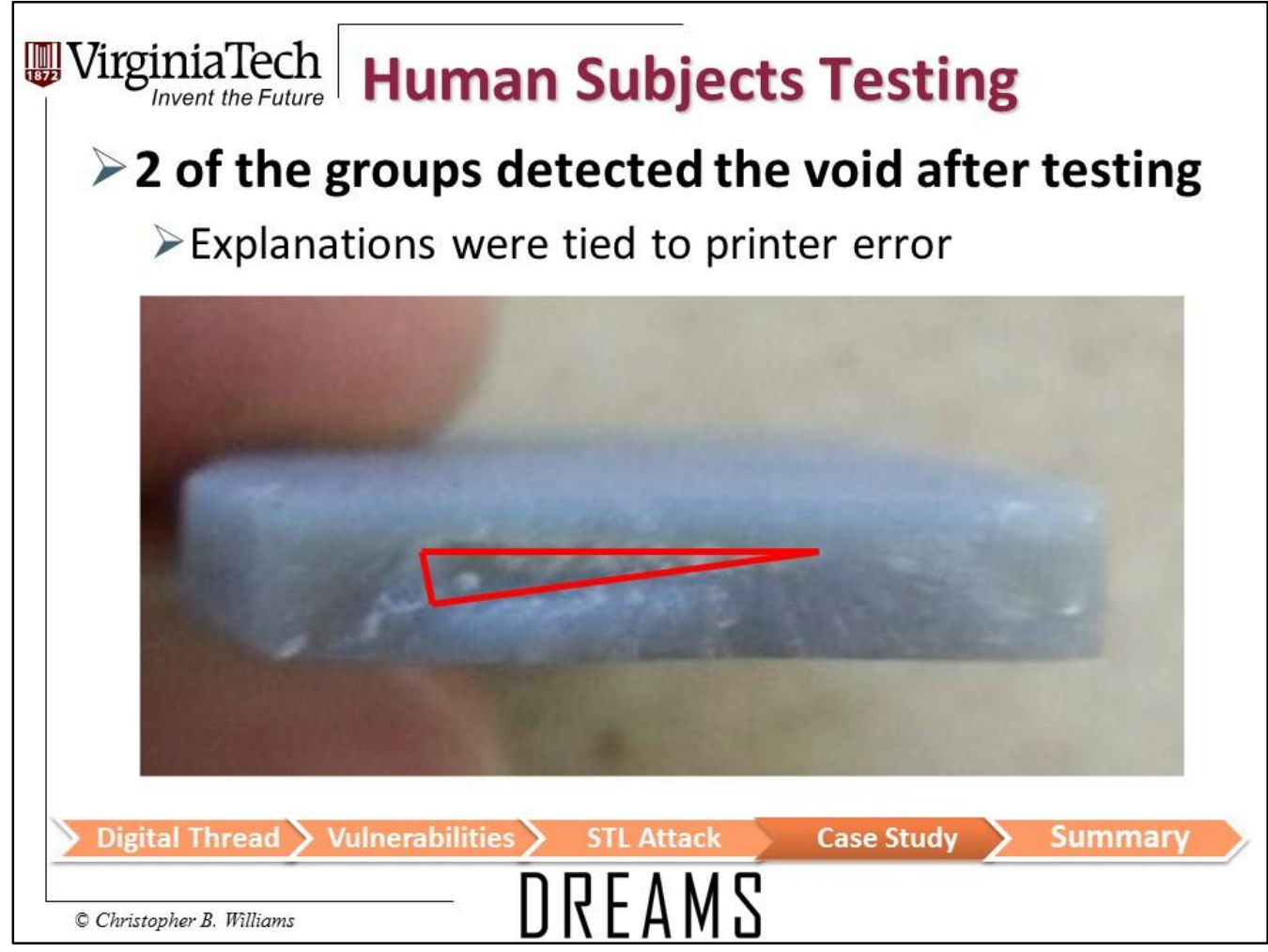

\section{WirginiaTech Prevention: STL Void Detection}

$>$ Determine the number of shells in a file

$>$ Files with only one shell cannot contain a simple void

\section{Determine shell size}

$>$ Shells with a small number of faces are likely to be nonfunctional parts

\section{Identify inverted shells}

$>$ Void normals will all be facing inwards

$>$ How to detect small features that are not separate shells? 


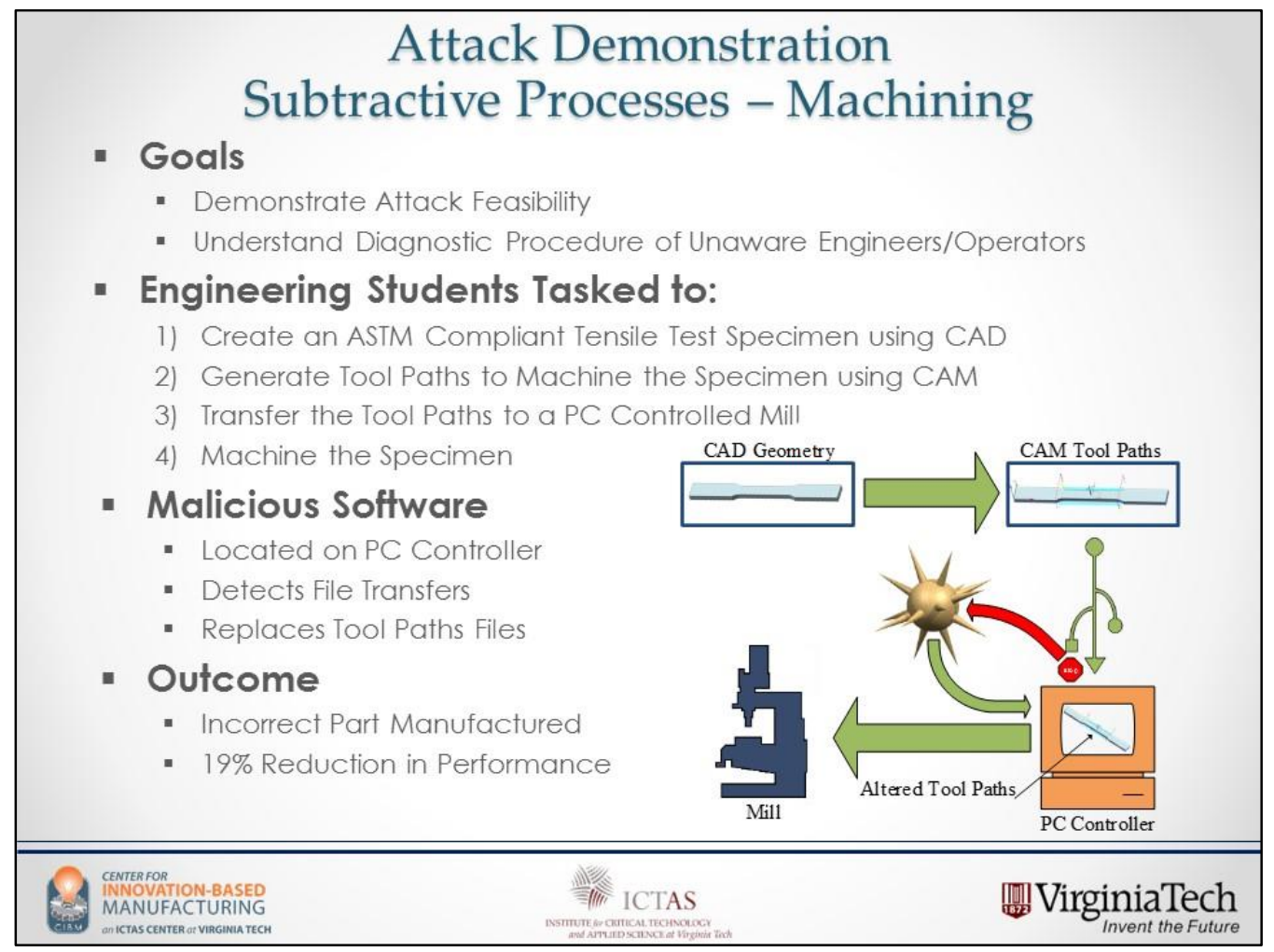

\section{Demonstration Results}

- 7 Groups attacked (3-4 students each)

- No group detected the attack

- First 3 groups did not measure the part

- Part "Looked Correct"

- Last $\mathbf{4}$ groups measured the part, identified as incorrect

- Diagnostic strategies

- Step-by-step: each step of the process was checked for errors

- Incorrect file was identified

- Step-by-step (reverse): each step (in reverse) was checked for errors

- No problem wasidentified

- Ordered: steps were checked for errors from most likely to least likely

- No problem was identified

- Correct diagnosis was never made 

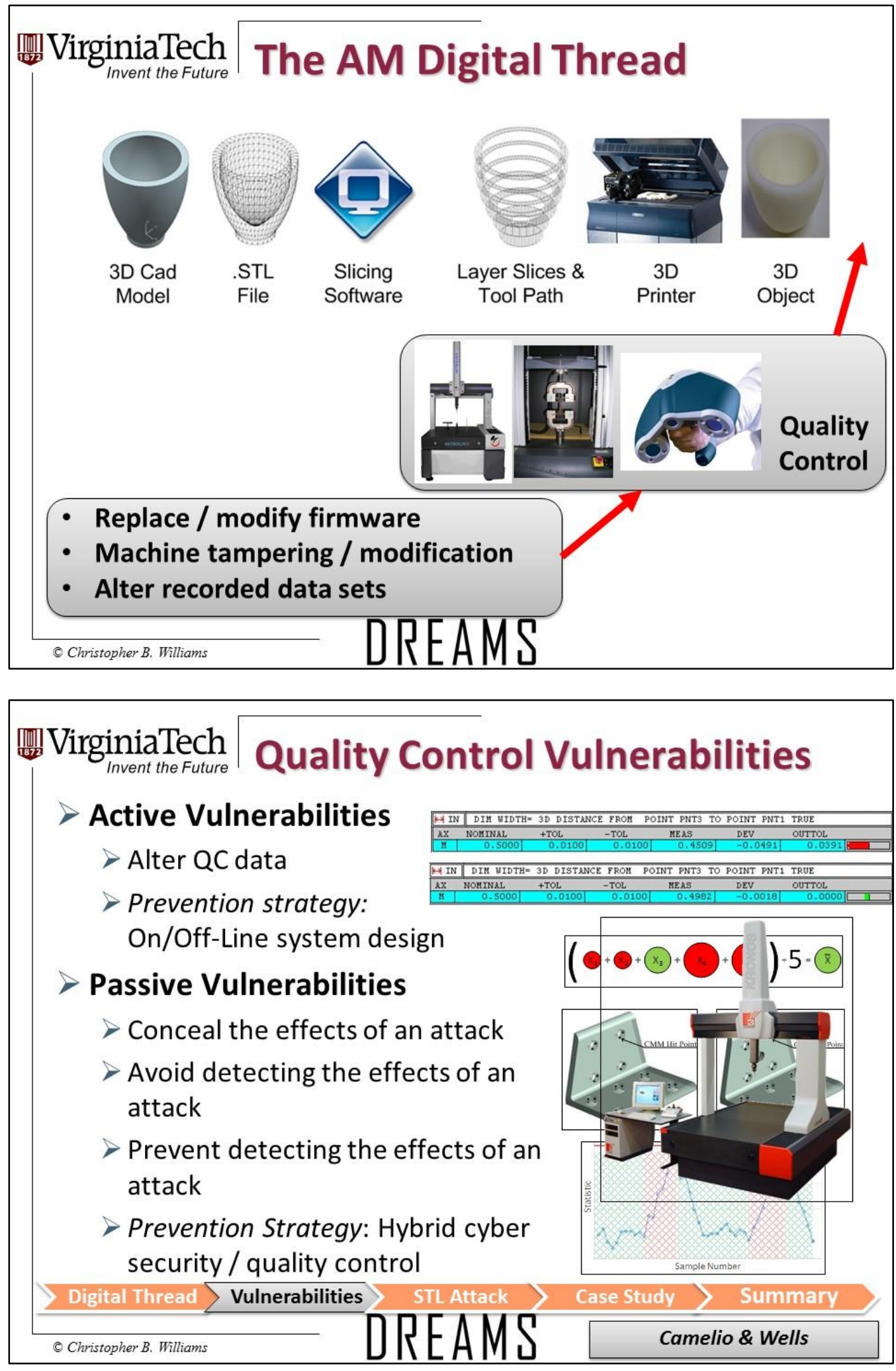


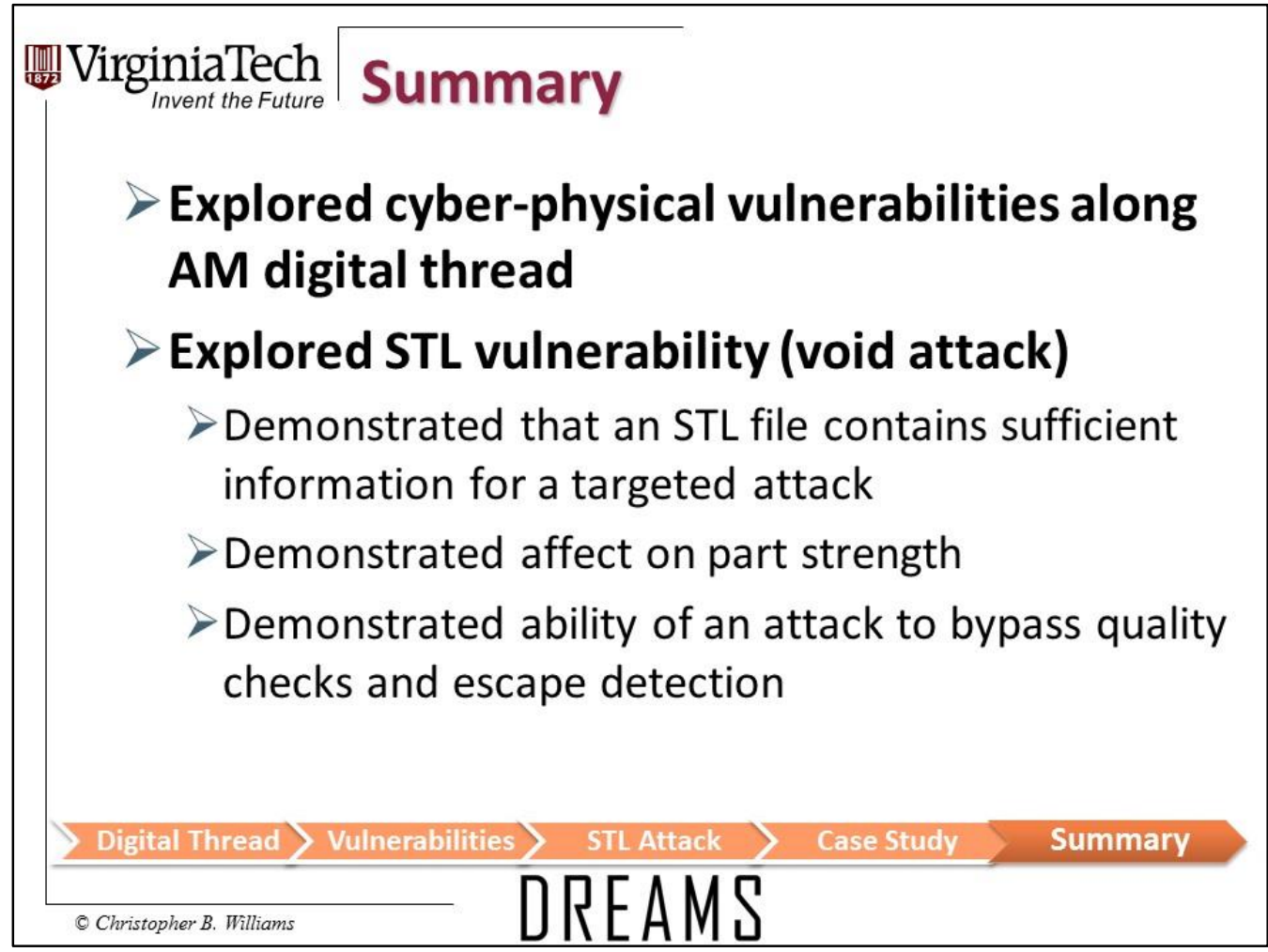

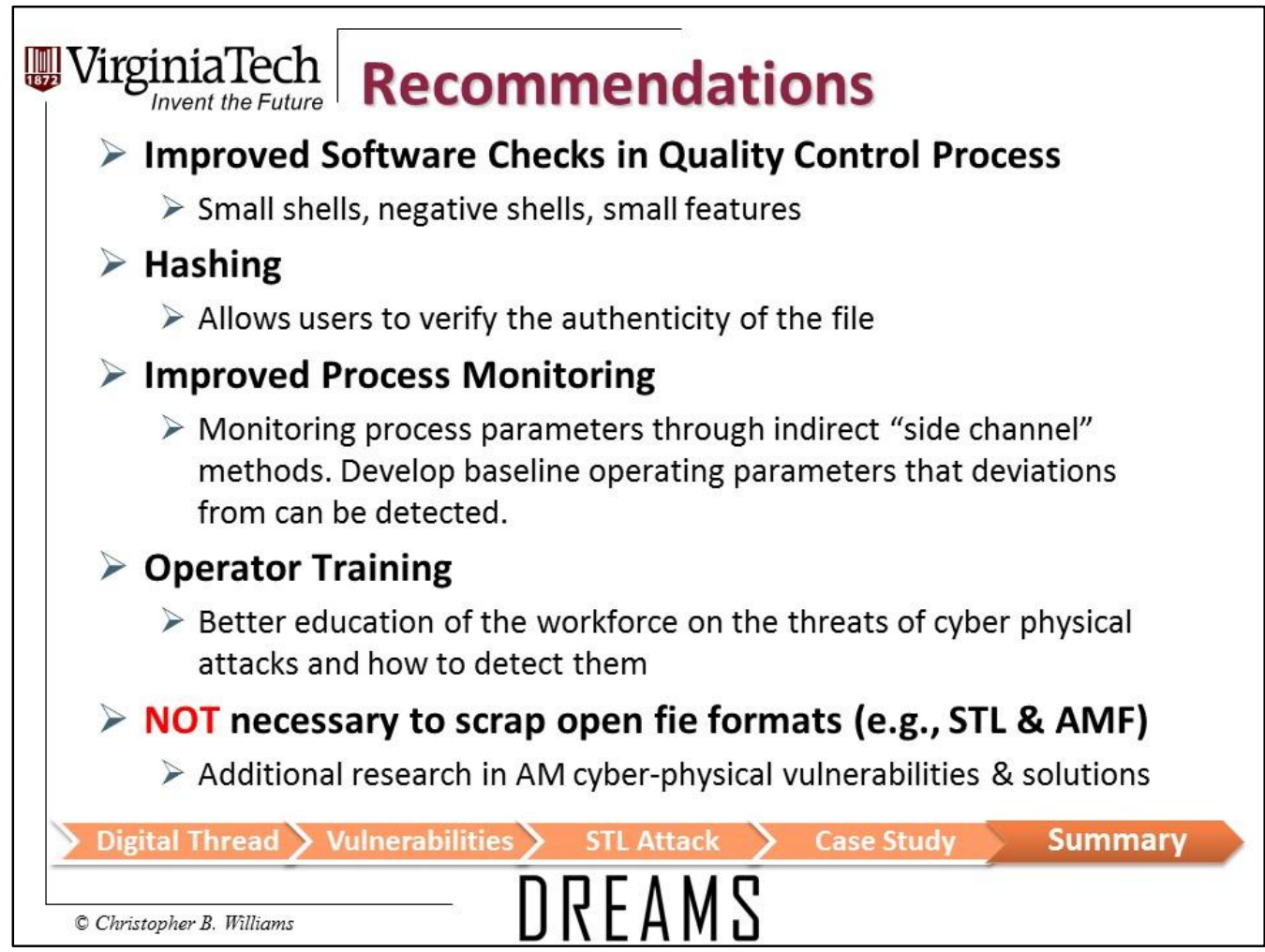



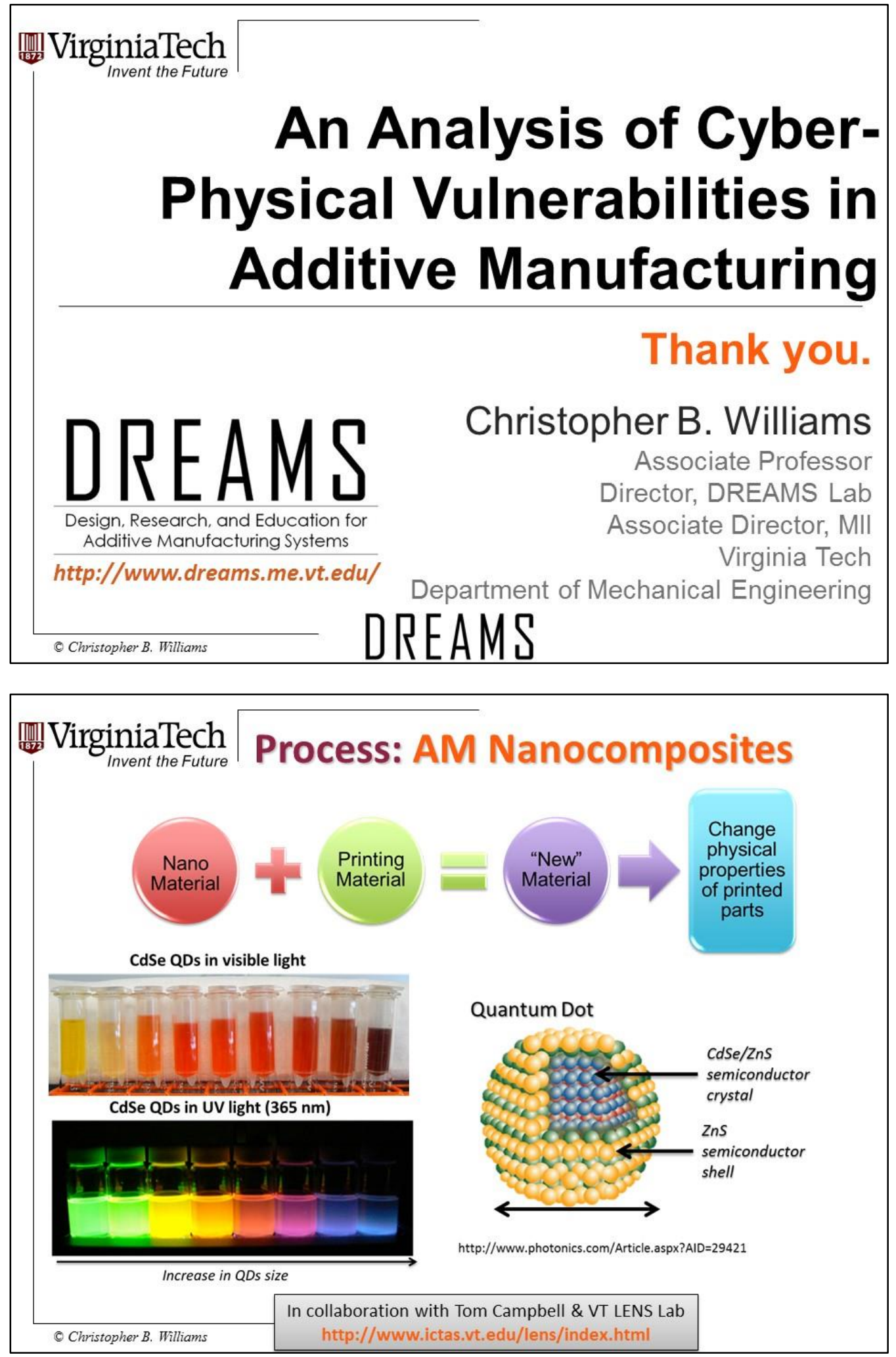


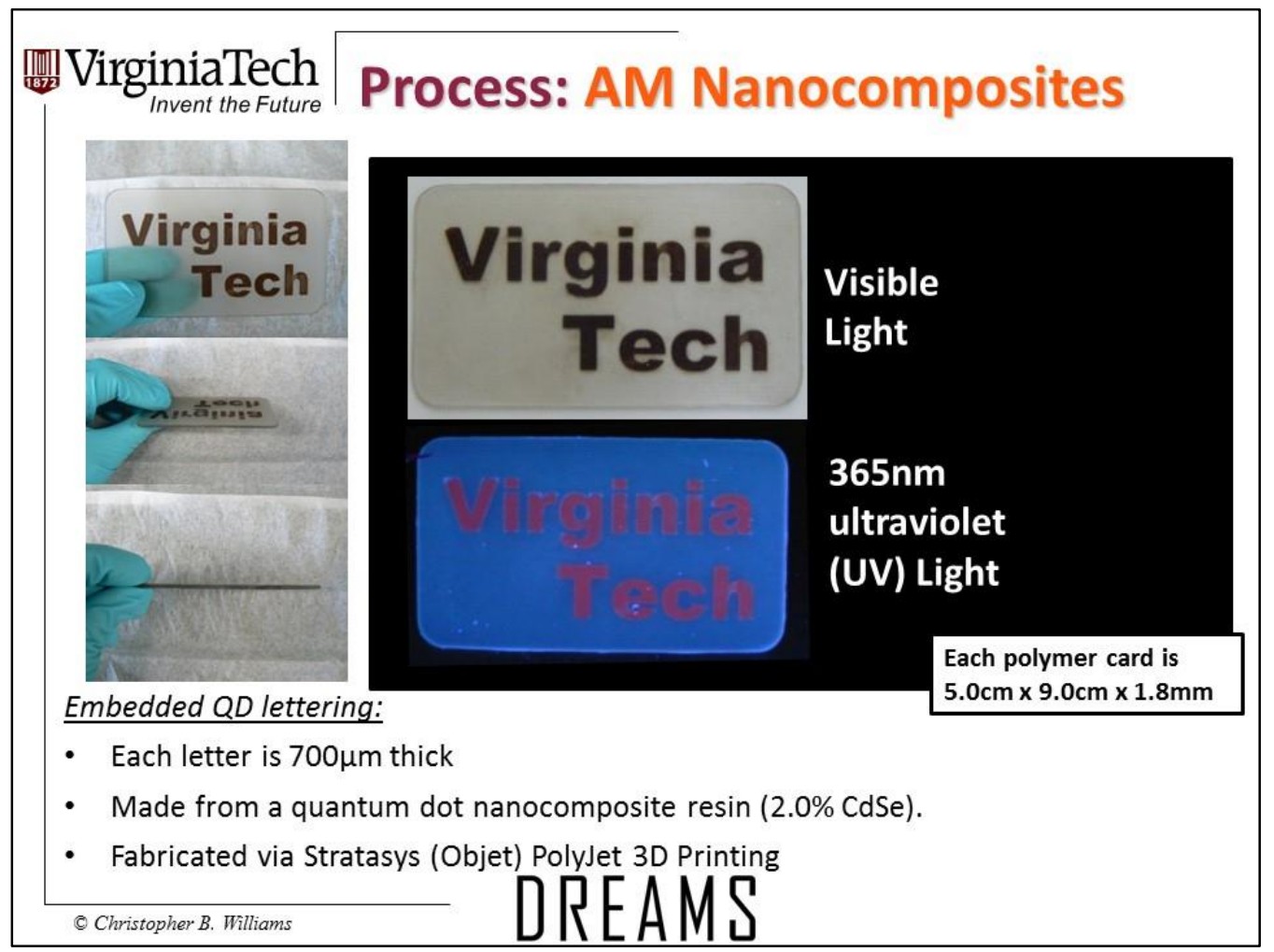

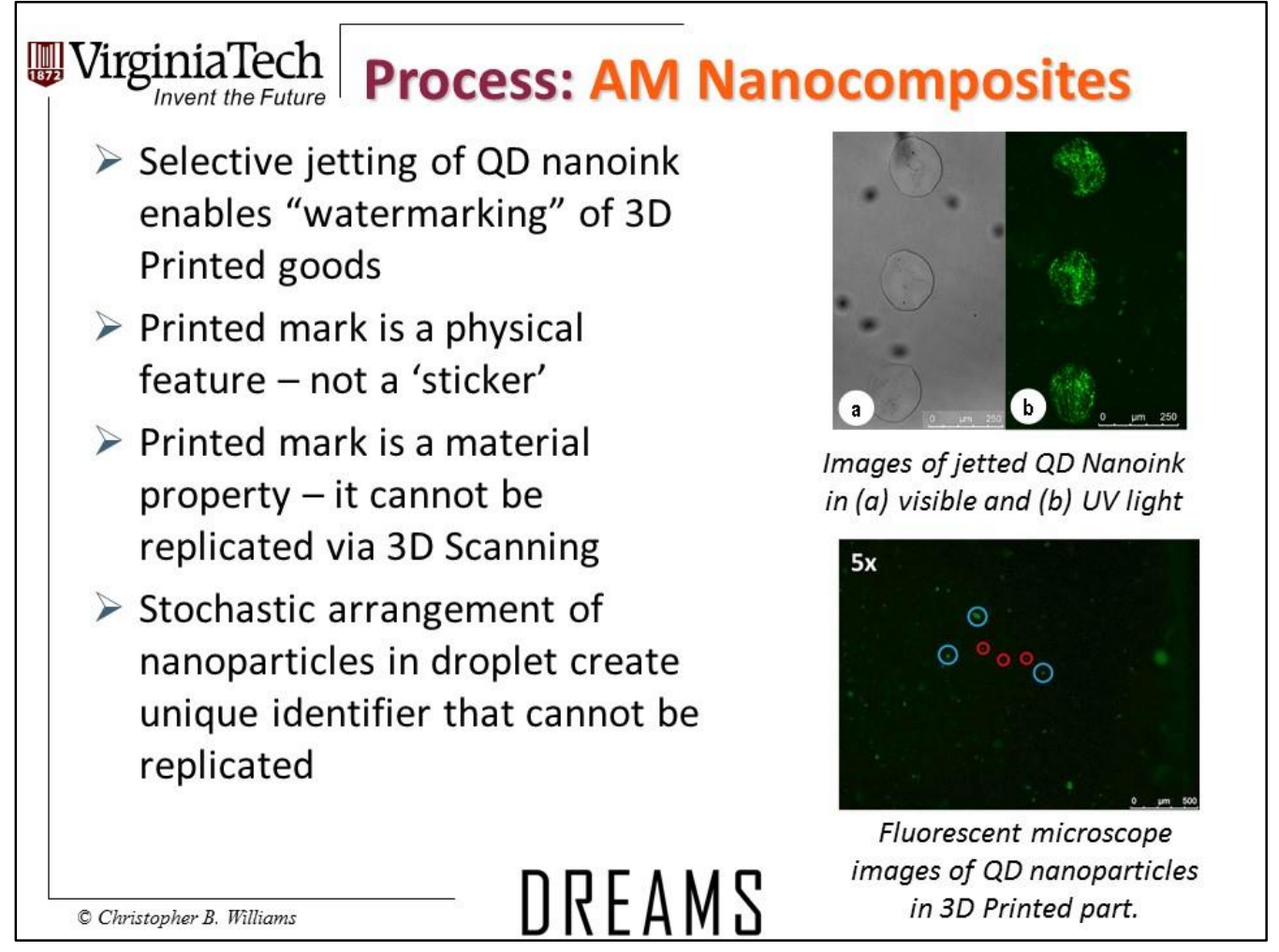




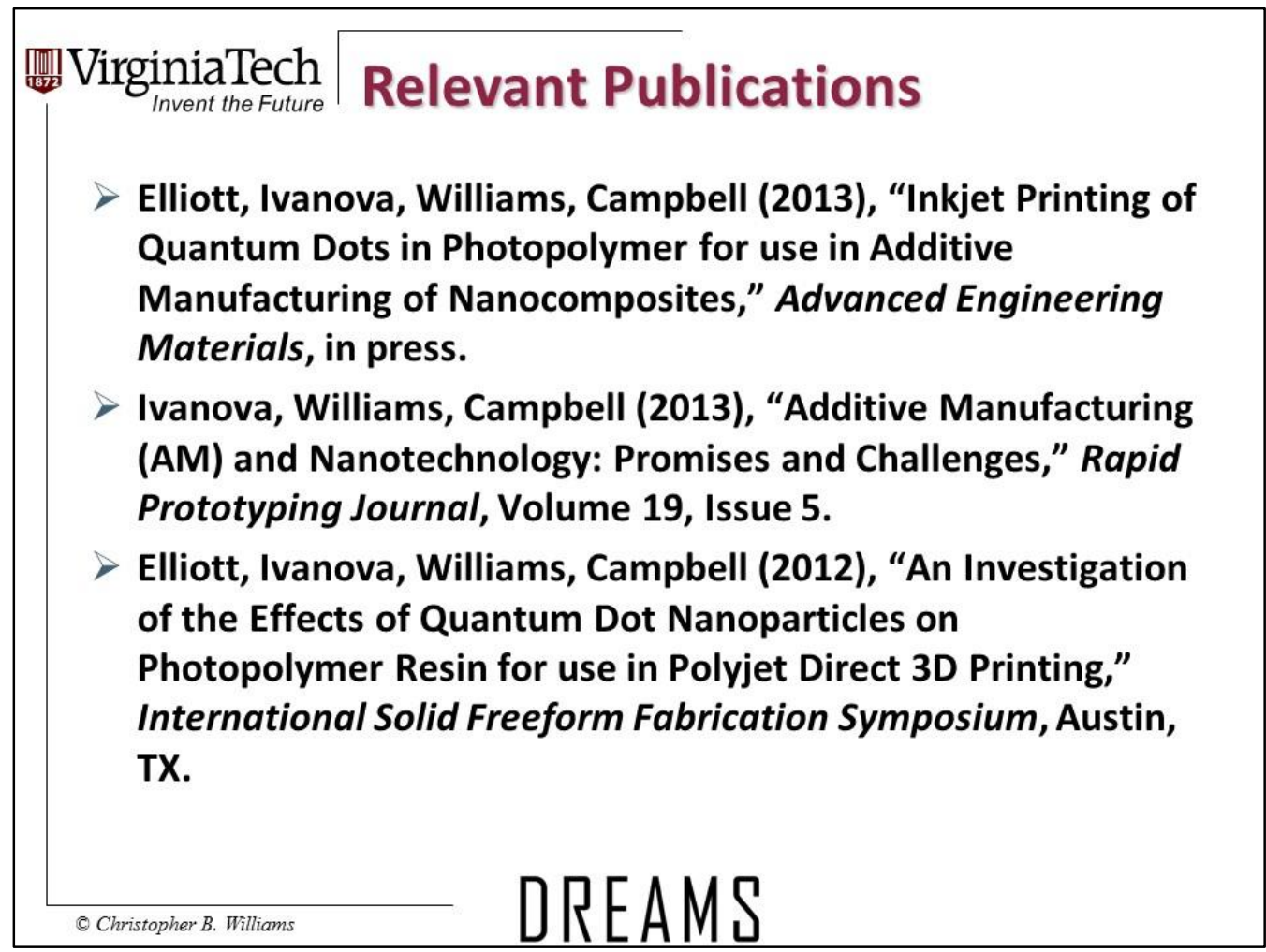




\section{Presentation 2: Applying and Assessing Cybersecurity Controls for Direct Digital Manufacturing Systems}

Scott Zimmerman, CISSP-ISSEP

Principal IT Advisor, Concurrent Technologies Corporation (CTC)

\section{Dominick Glavach, CISSP, GCIH}

Principle Fellow, Information Systems Security Engineer, CTC

Key Discussion Points:

- Digitization of manufacturing increases the risks for theft, disruption, and sabotage. There are vulnerabilities in preproduction software, data storage and data transfers, the StereoLythography (STL) file format, printer components, and engineering / production practices.

- The presenters discussed their experience with obtaining a 3D printer and the cybersecurity challenges experienced when setting it up.

- Many AM machines contain old firmware, cannot be patched easily, and have poor authentication processes. It was commented that this is not unusual for manufacturing systems.

- The AM process is also complex, variable / changeable, and tends to leave a lot of residual data in various places, making cybersecurity without interfering with functionality a challenge.

- There is a significant opportunity to be proactive rather than reactive regarding cybersecurity due to the nature of the technology and the state of the industry. The authors presented several recommendations for cybersecurity controls and highlighted the value of traditional cybersecurity controls such as firewalls.

- Participants stressed the need for focusing on people - a recent attack was described that began with a phishing scam. One participant commented that manufacturers and users are not security aware, yet DDM supports minimal digital knowledge - any security solution needs to be simple and usable. 


\section{Applying and Assessing Cybersecurity Controls for Direct Digital Manufacturing Sytems}

\author{
Scott Zimmerman, CISSP-ISEP \\ Concurrent Technologies Corporation \\ Johnstown, PA USA \\ zimmerms@ctc.com
}

\author{
Dom Glavach, CISSP \\ Concurrent Technologies Corporation \\ Johnstown, PA USA \\ dg@ctc.com
}

\begin{abstract}
Applying meaningful and assessing impactful cybersecurity controls are ongoing and significant challenges for the Direct Digital Manufacturing (DDM) Community. These issues will be significant as the technology moves into the mainstream manufacturing supply chain. This presentation will, therefore, address cybersecurity threats to DDM, including insight into potential attack scenarios and motivations, gained through direct observations. We will discuss the details of a security assessment performed on an Additive Manufacturing (AM) system used for rapid prototyping and complex part production within the defense industry. Protocols and associated recommendations for incorporating security best practices during system installation and subsequent operation will also be presented.
\end{abstract}

Keywords —additive manufacturing, cybersecurity, direct digital manufacturing, programmable logic controllers

\section{INTRODUCTION}

Based on the expectation and potential impact in revitalizing the U.S. and global manufacturing landscape, Direct Digital Manufacturing (DDM), including Additive Manufacturing (AM) and other similarly disruptive technologies, will have a significant impact on national security. According to the National Defense University, "The propagation of this technology has generated a host of national security considerations, which connect to broader economic and policy developments.... Additionally, the deployment of AM technologies in manufacturing will likely promote greater interaction between the national security community and the private sector, as businesses will be able to produce prototypes and sophisticated components more inexpensively and quickly than before." 1 While supply chain implications and benefits are numerous, cybersecurity remains a significant challenge.

The Economist (April 2012) refers to the potential for DDM to create the third industrial revolution ${ }^{2}$, noting that the disruption to manufacturing will be as significant as digitization was to telecommunication, office equipment, photography and publishing. While digitization creates an incredible growth potential within manufacturing, it also comes with many of the associated cybersecurity risks that impact other digitized industries.

Due to the potential economic and security implications of DDM, the industry is challenged to address cybersecurity risks in a timely way and develop standards, systems and processes for security before such wide scale adoption of the technology limits, or prohibits, the deployment of protection mechanisms. The negative impacts of failure to include security protocols at start-up can be seen within the power and energy sector, which has large deployments of programmable logic controllers (PLC) and supervisory control and data acquisition (SCADA) systems. At the time of design and deployment, these systems were not equipped with adequate security mechanisms to contend with the threats of the connected world in the current environment. Now these systems are so tightly woven into the fabric of the power grid, retrofitting security is a much larger task than if it had been tackled in the beginning. 


\section{CHARACTERIZING THE THREAT}

The technical advances and economic impact associated with the DDM revolution attracts an innovative and entrepreneurial audience. History illustrates that new technologies have a tendency to influence a criminal opportunity via unexpected exploitation avenues. From the stagecoach to smart thermostats, security has often been an afterthought in new technology design and implementation. Hathaway states that corporate and government leadership are reactive in nature to cybersecurity needs and only act to mitigate security issues after a significant event occurs. She further concludes that additional legislation may be needed to incentivize corporate and government leadership to get serious about cybersecurity. ${ }^{3}$

The complexity and critical nature of some products being produced by DDM, ranging from fuel nozzles to human organs, render these systems obvious targets for cyber criminals, espionage actors, or digital activist groups. Regardless of motivation, gaining access to an industrial DDM system is not a trivial action and requires an intricate, but likely, attack scenario, resulting in one of the following:

1. Theft (processes and property)

2. Disruption (slowing or stopping the DDM process)

3. Sabotage (inserting unforeseen time-delayed failures)

The combination of system complexity, installation methods and manner in which digital models become manufactured objects create a large attack surface. The proposed presentation explores possible attack scenarios and associated risk evaluations in the areas of:

1. Model file formats

2. Data storage and transfers

3. Printer components software and firmware

4. Preproduction software

5. Engineering and production practices

\section{SECURITY ASSESSMENT RESULTS}

\section{System Installation}

With the opportunity to conduct a security assessment on a newly installed AM system, we have identified risks at the inception; it begins with internal coordination and communications between enterprise Information Technology (IT) and shop floor personnel. In general, the focus and priority of the materials/manufacturing/engineering staff are installation and operation, which includes connection to the internal and possibly an external network, so the relevant parts can be produced. Their initial concerns are not about how to make this system secure.

In the particular case under consideration, the AM equipment was delivered to the 'manufacturing' floor, unboxed and set up all without the awareness of the IT department. Once installed, the AM engineering team connected with the Enterprise Help Desk and requested "...can you help connect our new printer to the network?" Unwittingly, the request was executed. Needless to say, the original equipment manufacturer (OEM) was unable to connect to the AM equipment, since it was behind the corporate firewall. Subsequent requests were submitted to the Enterprise Help Desk requesting OEM access to the equipment through the Internet for finetuning. The printer was transferred to an open Internet connection normally provided to corporate guests. This channel is monitored yet it has minimal shielding. It was only after subsequent investigation by the information security team that it became clear that the "printer" was in fact a metal DDM system, not a typical office document printer. Following this discovery, the security team has moved the printer to a secure and scrutinized subnet on the network. Now, additional security controls and enhanced logging occur routinely and yet where it is still possible for the engineering team to work directly through the network with the manufacturer.

\section{Assessment Methodology}

AM systems can be complex, consisting of several central processing units (CPU) and PLCs, operating systems, and applications (including both AM-specific ones as well as applications that support the user experience, such as web-browser and Portable Document Format (PDF) readers). The CPU/PLCs communicate via standard network protocols such as TCP/IP within the printer and then to a gateway interface for larger network access. The operating systems and 
applications on these controllers process design data to produce $3 \mathrm{D}$ components.

We deployed both the corporate security assessment methodology as well as the security risk assessment provided in the NIST DRAFT NISTIR 8023, Risk Management for Replication Devices. We will present and discuss specific scan results and findings. In addition, we will propose a series of security protocols as best practices for any DDM system implementation. We list a selection of possible solutions below and we will expand on the requirements for success in this presentation.

\section{Recommendations}

- Mandatory scanning (enumeration) of system prior to deploying to the network and disable all unneeded communications/system processes,

- Review of user accounts/groups on the system including their level of privilege and adjust accordingly,

- Removal of all unneeded applications installed on the system (browsers, readers, games, etc.),

- Enable host based firewall to allow communication via secure ports to know IP addresses for manufacturer communications (disable this connectivity when not in use)

- Processes developed for system updates/upgrades

\section{Conclusion}

High-end AM printers are expensive, highly calibrated machines, increasingly complex, and generally not 'plug-and-play' systems. With respect to the system discussed in this presentation, there has been a great deal of ongoing support from the OEM in order to optimize printer operational performance. This type of support requires remote connectivity to the system. When the manufacturer is a foreign entity, this situation compounds security challenges and complicates protocols due to the need to comply with International Traffic in Arms (ITAR) regulations that may prohibit collaborations. At a minimum, many security assessment protocols and mitigation procedures implemented typically for enterprise business systems should be applied or adapted for implementation and operation of DDM systems.

\section{REFERENCES}

C.M. McNulty, N. Armas, "Toward the Printed World: Additive Manufacturing and Implication for National Security," September 2012 Institute for National Strategic Studies, National Defense University, Defense Horizons

The Economist, "A third industrial revolution". Accessed November 2014, http://www.economist.com/node/21552901

M.E. Hathaway, "Leadership and Responsibility for Cybersecurity", Georgetown Journal of International Affairs, pages 71-80, March 2013. 
Presentation:

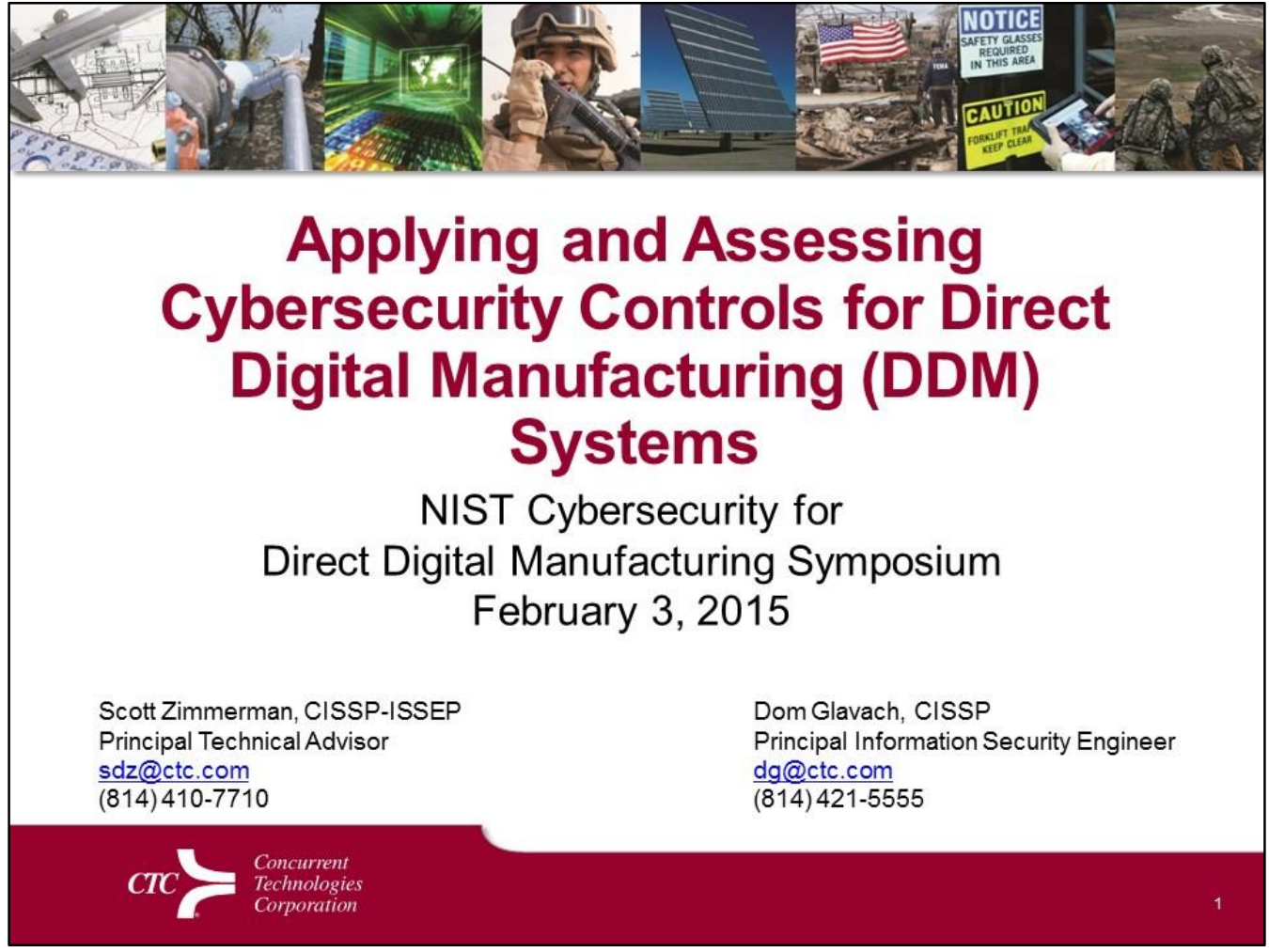

\section{Agenda}

- Introductions

- Defining the threat

- Attack scenarios

- Assessment

- Recommendations

- Conclusion 


\section{Concurrent Technologies Corporation (CTC) Summary}

- Established in 1987

- An independent, nonprofit, R\&D organization

- Staff of approximately 1,000 dedicated professionals

- Approximately 40 locations

CTC has been a thought leader in providing cybersecuritysolutions for over fifteen years. We deliverholistic solutions based on experience in protection, detection, and reactionmeasures as well as network engineering, management, modeling, and assessment activities. We provide both offensive and defensive capabilities. CTC's expertise in cybersecurity can be successfully applied to support cyberanalysis, improve situational awareness, increase operational performance, assure compliance with security policies, and address emerging technicalchallenges.
CTC $\begin{aligned} & \text { Concurrent } \\ & \text { Technologies } \\ & \text { Corporation }\end{aligned}$
Approved for Public Release

\section{Cybersecurity}

- Objectives of cybersecurity are to preserve:

- Confidentiality - protecting information from disclosure to unauthorized parties

- Integrity - maintaining and assuring accuracy and consistency of data over its entire life-cycle

- Availability - ensuring that authorized parties are able to access information when needed

- Measurement of cybersecurity

- Risk - potential for loss

- Threat - exploitation of a vulnerability

- Vulnerability - weakness in security

- Risk cannot be eliminated yet it can be managed and reduced

- DDM market presents new opportunities and attack vectors for those who wish to harm national security and for the criminal element

- Timely to build in and not bolt on security later

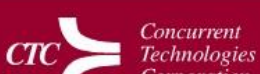




\section{DDM Cybersecurity}

- Digitization is as disruptive to manufacturing as it was to telecommunications, photography, publishing and other digitized industries

- No air gap now between enterprise information systems and shop floor

- Definite increase in attacks from information systems to critical infrastructure and cyber-physical systems

- Presents an opportunity to be proactive rather than reactive

- Develop standards, systems and processes before technology is adopted broadly when it limits or prohibits deployment of protection mechanisms

- From stagecoach to smart thermostats, security is often an afterthought in new technology design and implementation.

\section{DDM Cyber Risks}

- Theft

- Property, Process, License

- Disruption

- Process, Production, Equipment

- Sabotage

- Products, Reputation

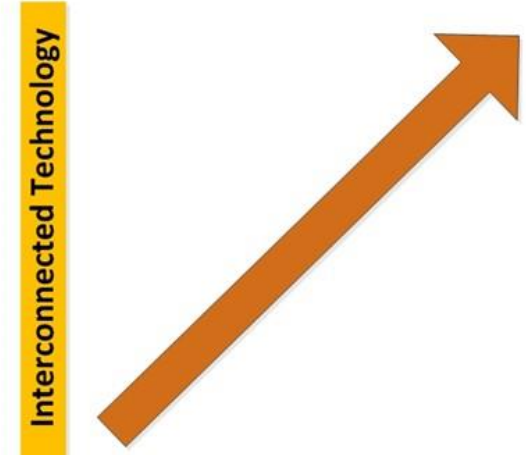

Attack Surface

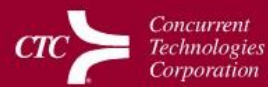




\section{DDM Cyber Threats}

- Who will attack?

- Sophisticated and funded, competitors, partners, criminals, state actors and terrorist elements

- Why attack?

- Economic advantage

- Military strategic and tactical advantages

- Political statements

$C T C \bigcirc$ Concurrent

Corporation

\section{Additive Manufacturing Digital Thread}

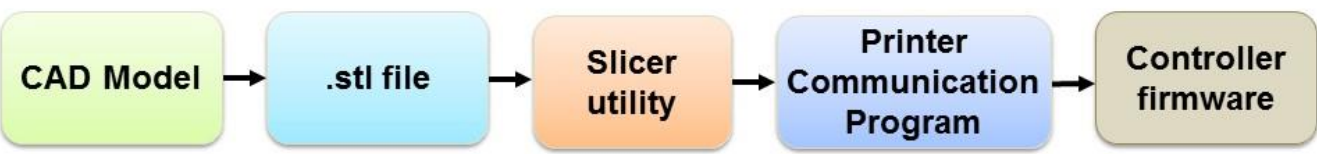




\section{DDM Cyber Vulnerabilities}

- Preproduction software

- Data storage and data transfers

- Model file formats

- Printer components

- Engineering and production practices

\section{Preproduction Software}

- AutoCAD

- 5 Common Vulnerabilities and Exposures (CVE)

- 2 enable unauthorized program execution

- 3 enable unauthorized access

- Trimble SketchUp

- 4 CVEs

- 2 enable unauthorized program execution

- 2 enable software disruptions (overflows) 


\section{Data Storage and Transfers}

- USB

- Uncontrolled media

- Historically known as an exploitation avenue

- Residual data

- Configuration management

- Network

- Enables automated attacks

- Increases complexity

- Multiparty access and access controls

\section{Model File Formats}

\section{STL files (STereoLithography)}

- "Trusted" standard in the industry

- Binary and plaintext formats

- Lack file integrity checks 


\section{Printer Components}

\section{Highly calibrated system with}

- Operating Systems

- Microsoft Windows and Linux

- Internet Explorer

- Camera software

- Firmware

- Control software

- Self contained network

\section{Engineering and Production Practices}

- Internet born STL examples

- Calibrate your printer.stl.exe

- Revision control

- $\mathrm{N}$ copies of intellectual property in $\mathrm{X}$ locations

- Residual data

- Everywhere

- Support and maintenance

- Remote access and remote control 


\section{DDM Case Study}

"Hey, can you install this new printer we just received?"

- AM system for rapid prototyping

Selective Laser Melting (SLM) Machine, SLM Solutions

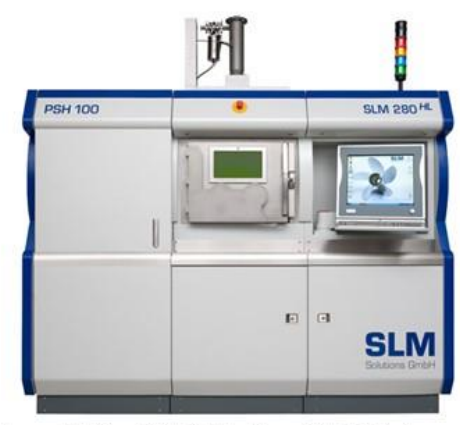

\section{DDM Cyber Assessment}

- Assessment methodologies

- CTC proprietary assessment methodology

- NIST DRAFT NISTIR 8023, Risk Management for Replication Devices

- Results

- Most applications and OS's unpatched

- Factory default install of AV/Host IDS

- No process for updating/patching

- Residual data left everywhere

- Poor authentication (shared/default passwords) 


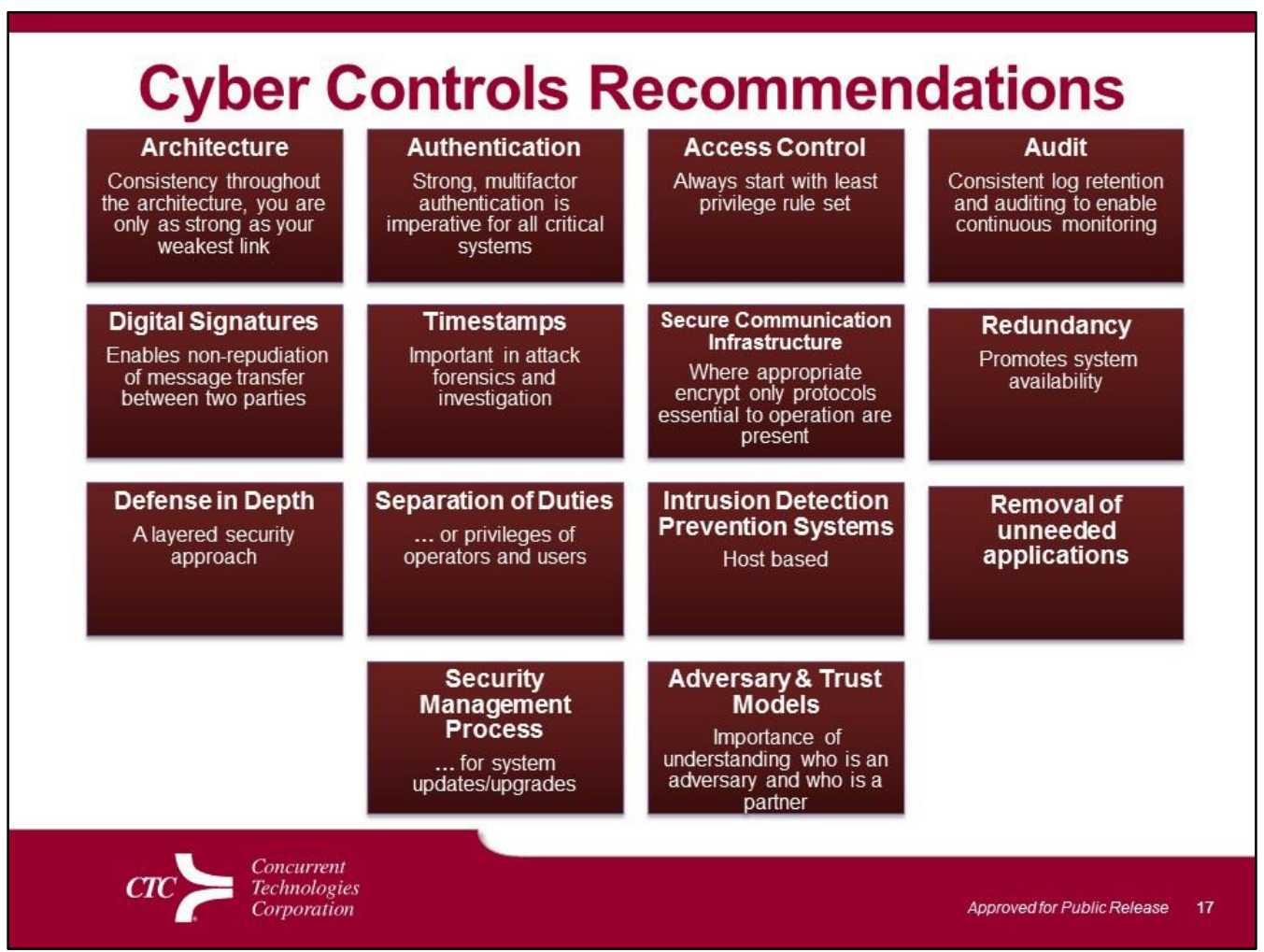

\section{Conclusion}

- DDM systems are ...

- Increasingly complex 'system of systems'

- Not 'plug and play', often requiring continued access and support by the (foreign) manufacturer

- At a minimum, security assessment protocols and mitigation procedures implemented for enterprise business systems should be applied or adapted for implementation and operation of DDM systems

- Consider security up-front and throughout all aspects of the equipment and process lifecycle (design through disposal) 


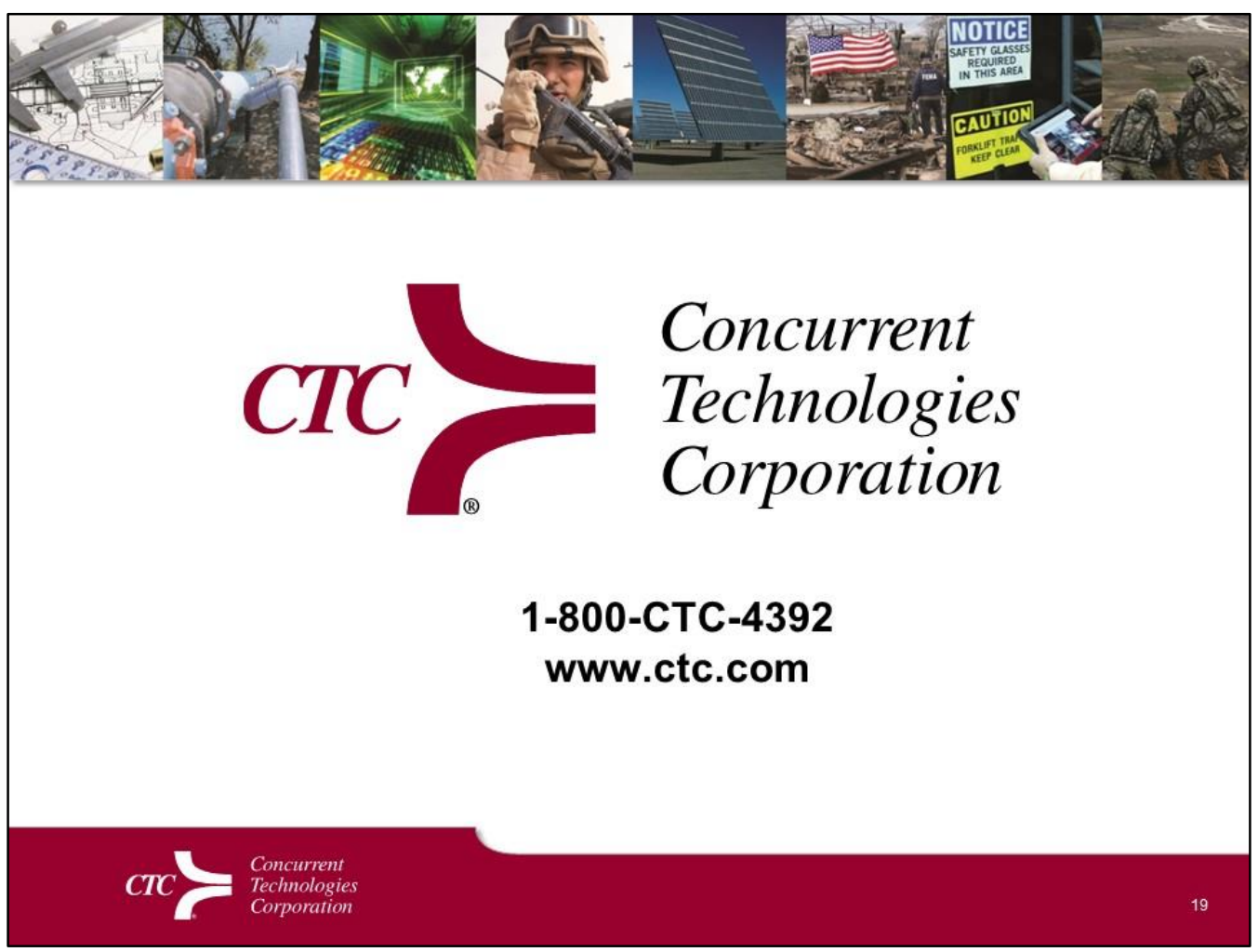




\section{Presentation 3: Cybersecurity for Advanced Manufacturing - Securing the Digital Thread}

\section{Dr. Michael F. McGrath}

National Defense Industrial Association (NDIA) Manufacturing Division

Key Discussion Points:

- The intersection between cyber/cybersecurity and manufacturing is critical.

- The presenter described three concerns expressed by manufacturers: theft, alteration, and disruption. These closely mirror the traditional Confidentiality, Integrity, and Availability (CIA) security objectives..

- IT solutions don't always fit the manufacturing world. Manufacturers often have a mix of old and new equipment. The new can be secured, but securing the old is much more difficult, and the old has to work with the new.

- Culture change is necessary. Some participants indicated the industry has to change - vendors will say anything to sell a product; manufacturing CEOs place productivity over security, and CISOs don't have much say regarding the manufacturing operations.

- Requirements are beginning to be seen - e.g. Defense Acquisition Regulations System (DFARS) clause which requires flow down of responsibility to subsuppliers.

- Some companies may be especially vulnerable as they may not recognize a risk. Interconnected supply chains with a lot of data sharing may be especially vulnerable if they use small company suppliers who don't recognize cybersecurity risks in manufacturing.

- Manufacturing presents a unique set of problems combining cyber plus Industrial Control System (ICS) vulnerabilities. Existing cybersecurity controls may not be sufficient in a DDM environment. The problem is not unique to AM, but AM presents a significant opportunity to build security in.

- An NDIA working group regarding cybersecurity in manufacturing is currently being formed. 


\title{
Cybersecurity for Advance Manufacturing -- Protecting the Digital Thread
}

\author{
Dr. Michael McGrath \\ National Defense Industrial Association (NDIA) Manufacturing Division \\ Arlington, VA, USA \\ mfm@mcgrath-analytics-llc.com
}

\begin{abstract}
Government and industry have focused much effort on protecting technical information in business and engineering information systems. Relatively less action has been taken to improve protection of technical data in factory floor networks and control systems, which are increasingly subject to cyber threats. NDIA's Manufacturing Division and Cyber Division jointly developed a White Paper in 2014 to heighten awareness of the need for better practices and technical solutions to protect against theft of technical data transiting or residing in manufacturing systems, alteration of the data (thereby compromising
\end{abstract}

the physical parts produced), or interference with reliable and safe production operations. Direct digital manufacturing is not inherently more vulnerable than other types of manufacturing, but it presents a very inviting target for would-be Intellectual property thieves or counterfeiters -- the full set of product and process information is available in one place, and the barriers to entry are low. This presentation offers several recommendations for enhancing protection of technical data in factory floor networks and in direct digital manufacturing systems in particular. 


\section{Presentation:}

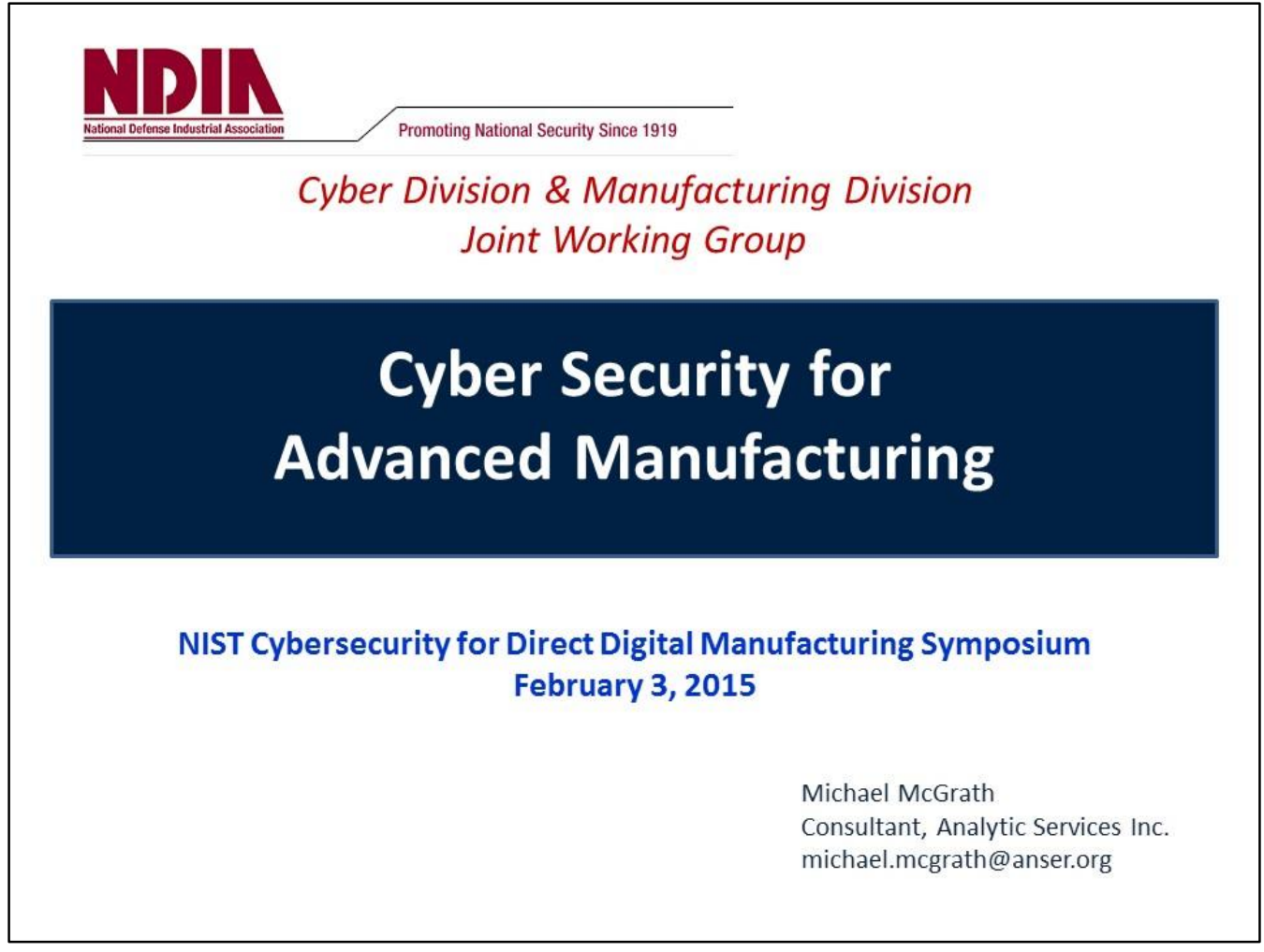

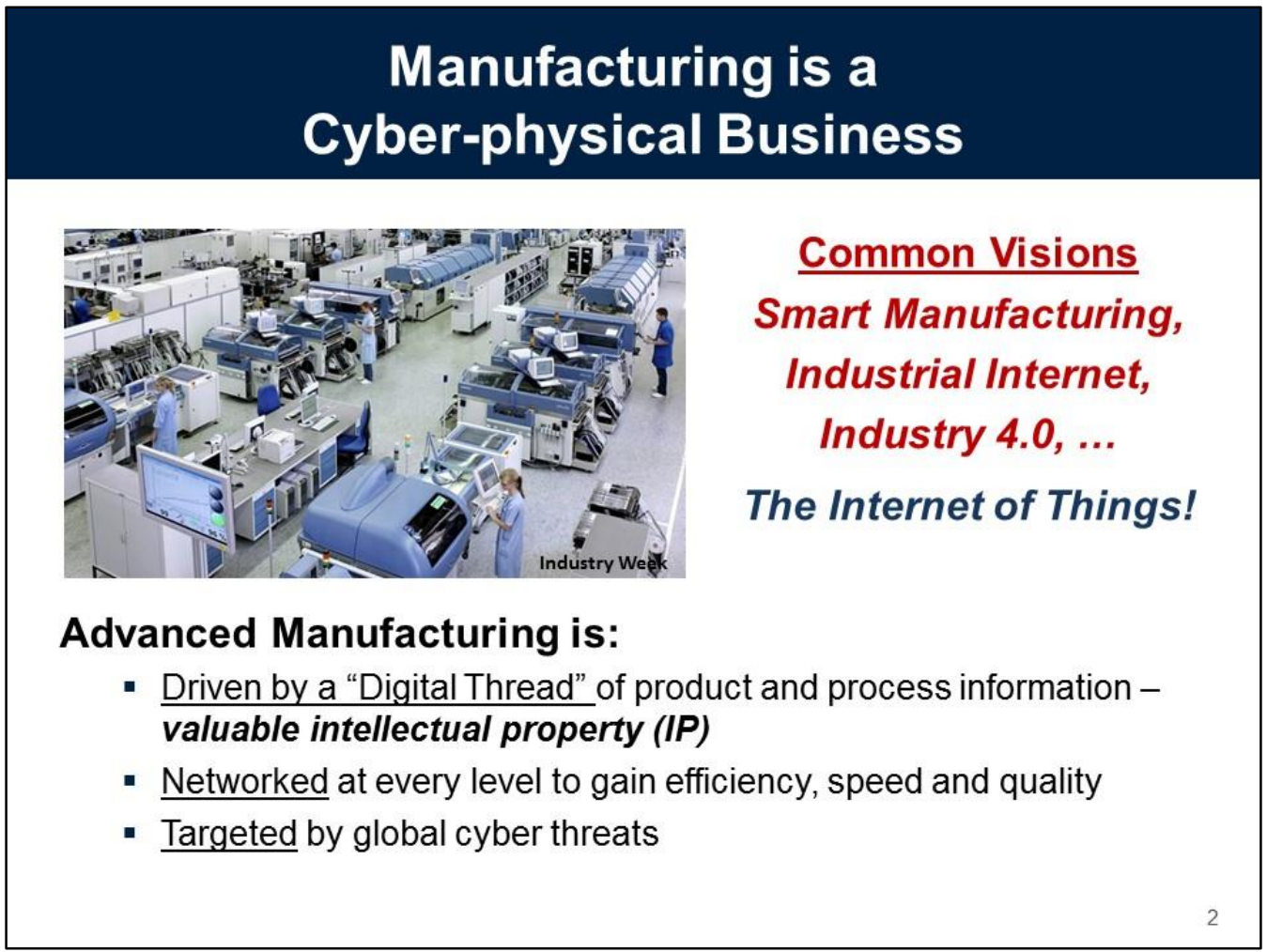




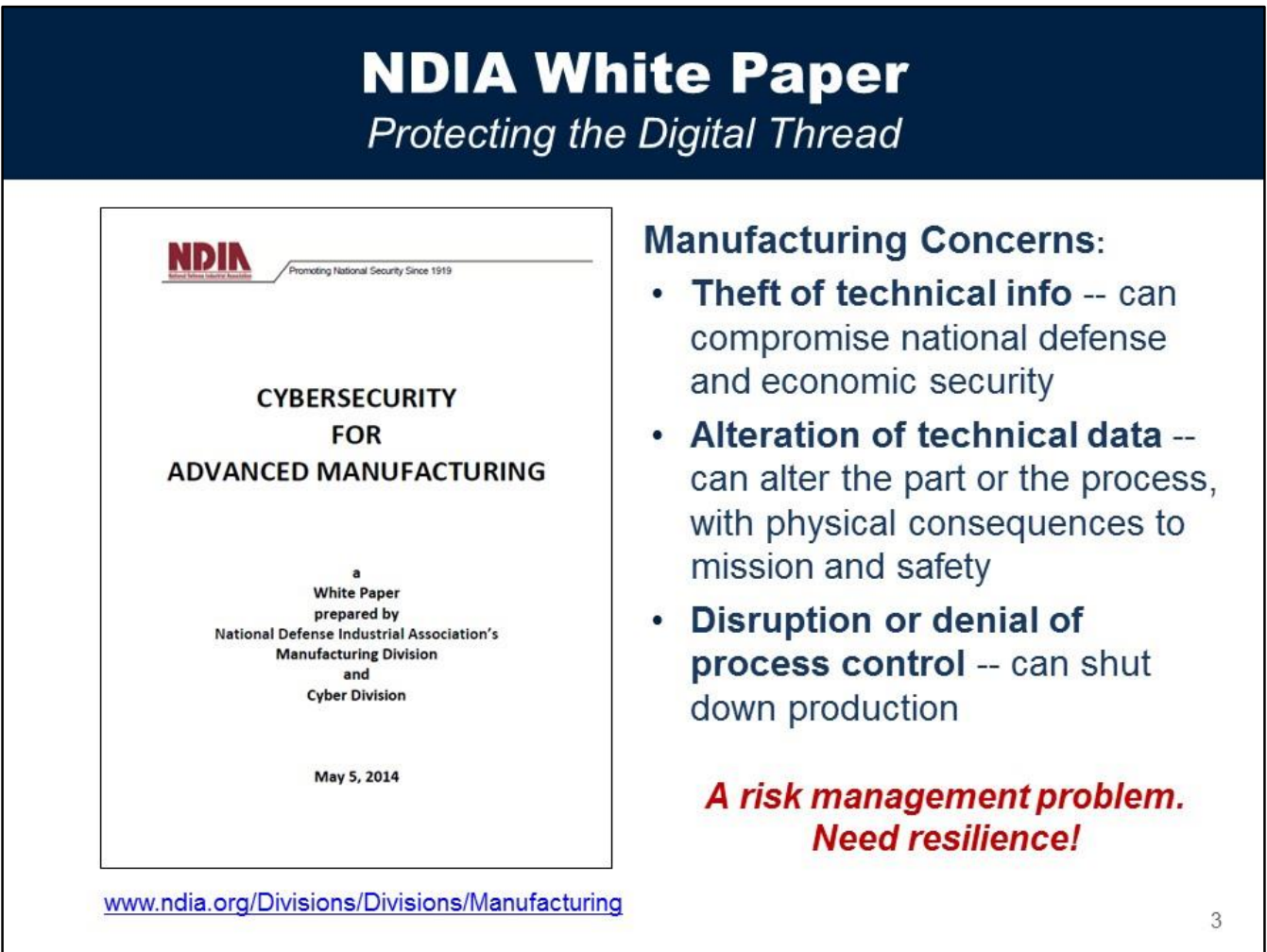

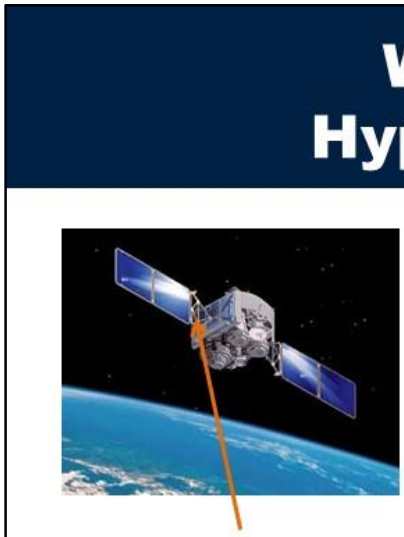

Use additive mfg to integrate power electronics with solar panel support structure

\section{We Started with a} Hypothetical Scenario

- OEM sends 3-D product data file to supplier for prototyping

- Intercepted by advanced persistent threat

- Prototype fabricated, delivered and successfully tested

- OEM sends new file, order for 12 parts

- Intercepted and modified by adversary to include a kill switch. Not detected.

- Parts fabricated, QC'd, delivered and installed

- Bird on orbit with kill switch controlled by adversary

We quickly learned there are plenty of real scenarios to learn from ... 

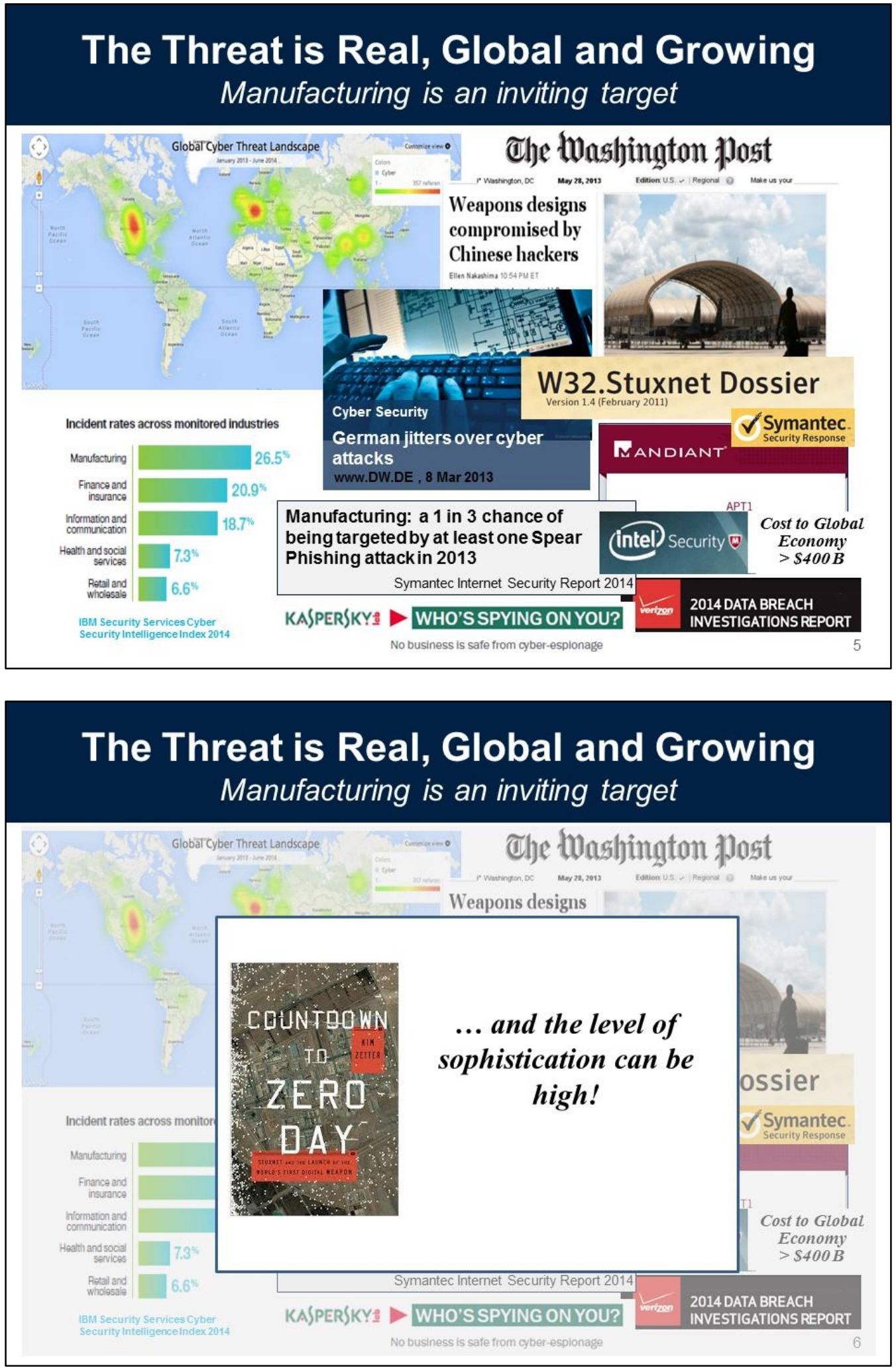


\section{Scope: Protecting the Digital Thread}

\section{Technical Data in the Advanced Manufacturing Enterprise}

Targeted by nation states, terrorists, criminals and hacktivists.

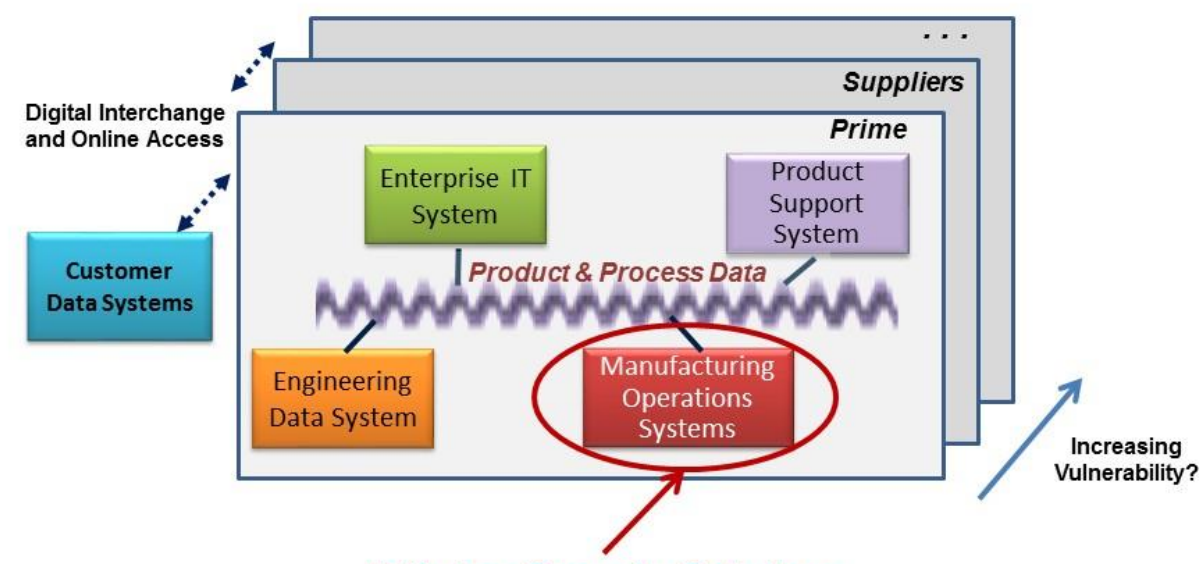

IT Cyber Security Solutions

May Not Fit Manufacturing Operations Needs

\section{Operational Technology (OT) vs. IT}

\section{What's Different?}

- ICS systems are long-lived capital investments (15-20 year life)

- Obsolete operating systems and software are common

- New systems architected for security, but hard to interoperate with old

- "Production mindset" with little tolerance for OT down time

- Operate in real time with critical safety implications - cannot install patches without scheduled downtime and testing

- Weak privilege management among operators and maintainers. Growing use of wireless devices.

- Nascent cybersecurity awareness and limited workforce training.

- Manufacturing differs from other ICS applications (e.g. Power Grid)

- Every manufacturing job brings new executable code into system

- Tech data flowing through the system is a target 


\section{What We Heard from Interviews} Gov't, Industry, Academia

- $\mathrm{ClOs} / \mathrm{ClSO}$ in the defense primes are implementing strong cyber risk management and sharing info through the DIB CS/IA and DSIE programs

- Concerned about suppliers and willing to work with them

- Have not yet seen threat to factory systems, but acknowledge the possibility

- Need cost/risk tradeoffs to arrive at an affordable solution

- Industrial Control Systems (ICS) may be soft targets. Culture differs from IT.

- Standards and guides* for ICS provide good risk management approaches. Implementation is spotty.

- DoD has mandated protection of critical information

- Primes address in the program protection plan, but ICS security is not emphasized in DoD guidance

- Defense R\&D for cybersecurity is not currently focused on factory floor vulnerabilities and solution needs

\section{Recent Trends}

- Threat Sophistication Increasing

- Cyber as an Instrument of State Power

- Know-how adopted by cyber-criminals

- Attack Surface Increasing

- IT and OT convergence, wireless connectivity

- Internet of Things (est. 50B devices by 2020)

- Additive Manufacturing, ease of counterfeiting

- Mitigation Approaches -- Beyond Perimeter Defenses

- New network appliances for dynamic analysis

- NIST Special Pubs updates (e.g. 800-53, 800-82)

- NIST Framework for Improving Critical Infrastructure Cybersecurity

- DFARS 252.204-7012, Safeguarding Unclassified Controlled Technical Information

In our tests, attackers got through organizations' cyber Maginot line at least 97 percent of the time." FireEye/Mandiant, 2014 


\section{Contrast in Risk Mitigation Approaches}

\section{Commercial}

NIST -- Framework for Improving

Critical Infrastructure Cybersecurity

- Risk management framework

- Best Practices

- Informative References*

- Voluntary compliance and tradeoff analysis

* Multiple guides and resources (ISO, ISA, NIST, ...)

\section{Defense}

DFARS - Safeguarding Unclassified Controlled Technical Information

- Minimum mandatary cybersecurity controls*

- Prompt incident reporting and damage assessment

- Mandatory flow down to lowest level of supply chain

- Contractual compliance

${ }^{\star}$ From NIST 800-53

\section{NDIA White Paper Summary}

\section{Findings:}

- The threat is real and manufacturing companies are targets

- Factory floor systems are a weak link in safeguarding tech info

- Smaller manufacturers are not well equipped to manage the risks 


\section{NDIA Recommendations for DoD USD(AT\&L)}

1. Work with industry on risk-based, voluntary standards and practices for factory floor cybersecurity.

- Evaluate NIST framework as starting point.

2. Conduct forums with industry to help understand and implement DFARS clause, including factory floor implications.

3. Update DoD guidance on the Program Protection Plan (PPP) to include protection in factory floor systems.

4. Use red teams to expose vulnerabilities and R\&D to fill gaps

5. Assist SME suppliers with training and investments

- NIST Manufacturing Extension Partnership to deliver training

- Defense Prod Act Title III and Manufacturing Technology investments

- Training for DoD contracting officers

\section{Observations re: Additive Manufacturing}

- Growing Importance to National Defense

- Examples -- cooling ducts in the F-35, fuel nozzles for turbine engines.

- Ability to produce small quantities efficiently (lot size of one) is particularly attractive for DoD small production runs and spares.

- Therefore an Inviting Target

- The digital product/process file is valuable intellectual property for espionage, counterfeiting, or tainting parts.

- 2013 experiments by Virginia Tech - AM is a soft target. Easy to alter the the manufactured item in hard-to-detect ways.

- Window of Opportunity for Improved Security

- Inherently no more vulnerable than other manufacturing methods

- Now is the time to build more security into these emerging systems 


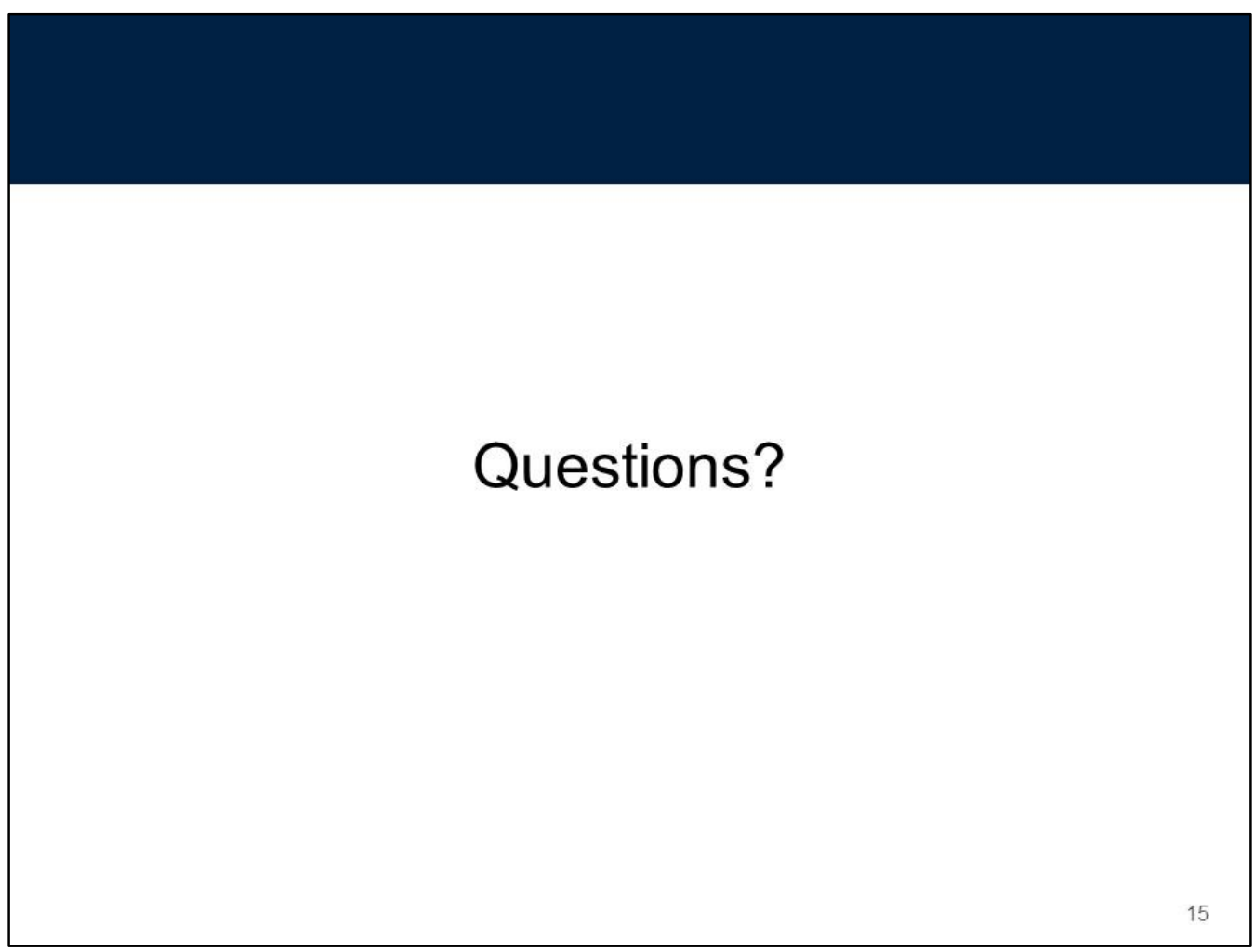




\section{Panel: Opportunities for Secure 3D Printing}

Robert Zollo (moderator)

President, Avante Technology

Dr. Claire Vishik

Trust and Security Technology and Policy Director, Intel Corporation

Andre Wegner

Founder and CEO, Authentize

Key Discussion Points:

- There are many opportunities for building security into the design of DDM machines abound.

- During its development, security wasn't high on the list of priorities for the ISO Additive Manufacturing File Format (AMF)[3], but it has "hints" of security there is a space in the metadata where security could be inserted. In the future, it may be added in.

- The Cyber Physical Systems (CPS) Public Working Group (PWG) considers manufacturing devices like 3D printers as cyber physical systems. AM devices are similar in that they use the same protocols and firmware.

- There are privacy concerns when considering cybersecurity controls. For example, putting in automatic, machine-generated ID numbers for asset inventory or forensic purposes could lead back to a particular printer and a particular person.

- One of the biggest impacts of AM may be on the supply chain. Distributed manufacturing with localized production can dramatically reduce logistics costs. AM provides an opportunity to enhance the resilience and security of the supply chain in ways not available before.

- The biggest obstacles to cybersecurity in manufacturing include: awareness; the culture; uninformed decision makers; loss of process control; people and organizations not working together; not willing to invest in security.

- Attendees disagreed as to whether the economy would need to provide an incentive for organizations to include cybersecurity in their processes. Some attendees stated that customers desire more secure solutions to protect their intellectual property and systems. Other attendees disagreed but were uncertain whether the market could be incentivized to be proactive or if solutions would always be reactionary.

- Attendees and the panel stated that there were no on-going activities regarding security standardization. It was noted that standards reduce costs significantly in the semiconductor and other fields, but the standards processes around AM devices have just begun and attendees were unsure how security standards could be applied. 


\title{
"Virtual Part" Perspective on Cyber-Security
}

\section{Designing Security Components into 3D Printing Hardware, Software \& Printed Objects}

\author{
Robert Zollo \\ President \\ Avante Technology, LLC \\ Bellevue, WA USA \\ bobz@avante-technology.com
}

\section{INTRODUCTION}

The author will provide a "ground up" view of security issues from the printer hardware and related control software perspective, and introduce the concept of the "virtual part", a term for the software and meta data that define the item to be printed, and its revisions as it moves and evolves throughout it's life in the integrated supply chains of future factories.

He will provide insight on how to employ the new ISO/ASTM standard for 3D printing file descriptions to begin building security components within the file meta data and use it with security functionality that can be designed in to the printer firmware and control software. He will propose some simple steps to begin building a cyber-security capable environment on the shop floor and in the engineering lab.

\section{THE "BRILLIANT FACTORY" CONCEPT}

A brief overview of the integrated "brilliant factory" of the future as described by GE in their recent white paper on DDM. The concept of integrating thousands of intelligent machines located in multiple locations by people within and without the manufacturing organization in a "completely transparent supply chain" is introduced. Security issues relating to the "virtual part" as it moves through the supply chain to the factory floor and back for revisions are highlighted.

\section{THE "STATE OF THE PRACTICE"}

A brief overview of some typical 3D printers will be offered to highlight areas of potential breach of security in the firmware, controlling software and the file description software. Opportunities for introducing simple security measures are identified.

\section{LEVERAGING ISO STANDARDS}

An overview of two ISO standards relating to the definition, transfer and use of 3D files is provided. Ideas on how these standards may be used to begin building some security mechanisms into the "virtual part" package as it moves through the design and supply chain.

\section{INTEGRATING SMALL SHOPS FOR SECURITY}

Suggestions are made on how to implement a simple, scalable, integrated security mechanism using components embedded in the printer firmware, control software, file management software, and file description software that is applicable to small to small manufacturing shops as well as enterprise scale brilliant factories.

\section{INVITATION TO DIALOG}

Panelists will be invited to comment on how the suggested security mechanisms might fit within a larger scale security architecture in enterprise factories.

\section{REFERENCES}

1. M. Annunziata and S. Biller, "The Future of Work", General Electric white paper; 2014.

2. ISO/ASTM 52915 standard framework for an interchange format to address current and future needs of additive manufacturing; 2013

3. ISO IS14306 standard for viewing and sharing lightweight 3D product information: 2012. 
Presentation by Robert Zollo:

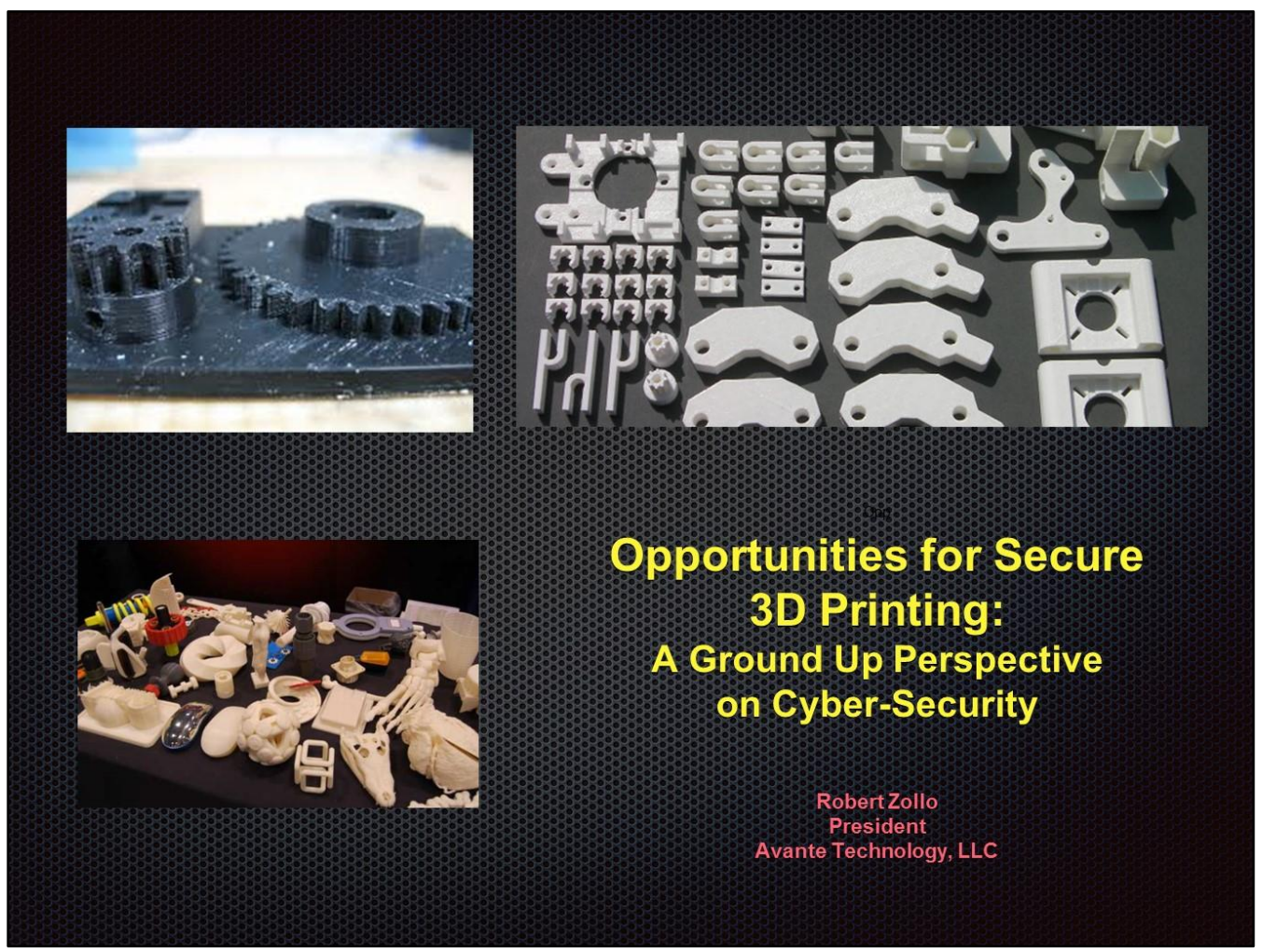

\section{Presentation Goals}

1. Introduce the Briliant Factory Concept

2. Identify Opportunities for Adding Security

3. Ideas on Leveraging Existing ISO Standards

4. Propose Ground Up' Steps for Secure Printing

5. Initiate Dialog among Stake Holders 


\section{What We Do}

- Design 3D Printing Systems

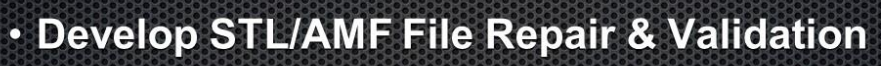
Software

:Develop Propietary Materials for FDM Printing

:Install Gustom Printing Solutions

$\because$ Technical Consulting

\section{A Slightly Different Perspective on Cyber-Security}

- Ground Up View from the Shop Floor

- Start with most basic 3D Printing Machine

- Identify Simple Practical Approach

-Utilize Basic Secuirity Building Blocks

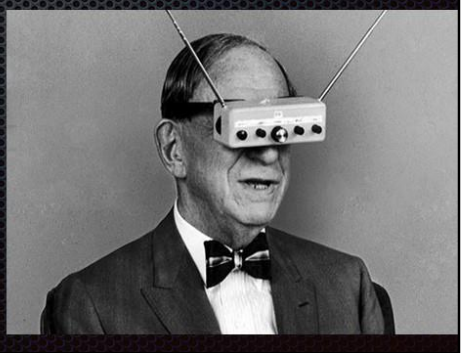




\section{Brilliant Factories of the Future Are Here \& Now}

A New Kind of Industrial Company

GE Eyes Growth by Linking:

- Machines

- People

- Data

\section{GE's Concept}

The dea behind the Briliant Factory

Link design, engineering, manufacturing, supply chain distribution \& services into one intelligent system 


\section{Eating the Proverbial Elephant}

How Do You Secure a Massive, Dynamic Matrix of this nature?

Or even a small one?

Break DoWhis Enomous Challeng into Small digestible bites of Security Components

\section{Security Starts in the Physical System:}

- Printer Hardware

- System Firmware

- Control Software

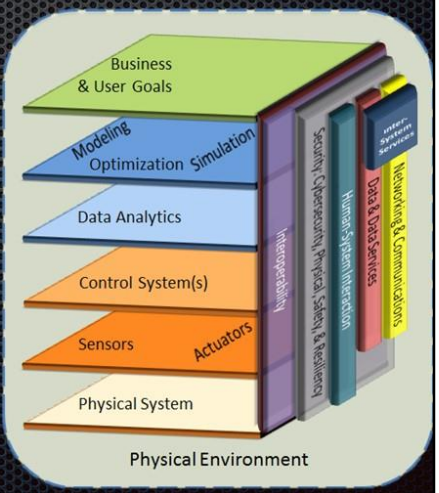




\section{Security Starts in the}

Physical System:

From Engineering Desktop

to Factory Floor...

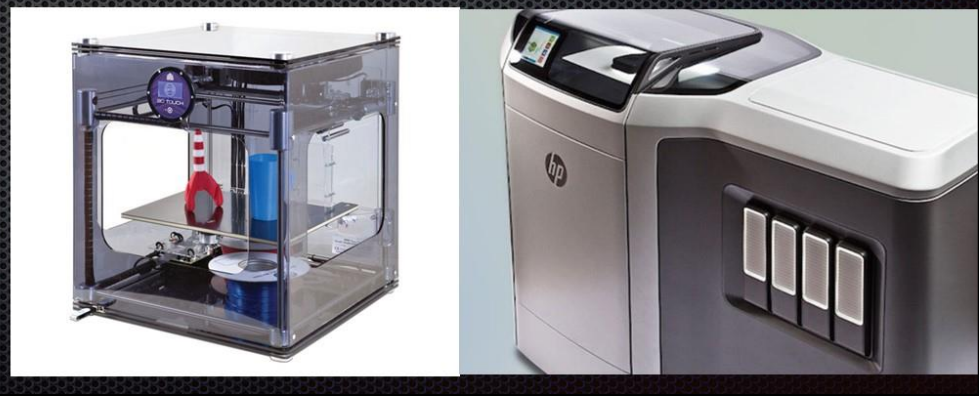

Security in the Printing System Hardware

Physical Locks:

access to control pane

access to memoy

access to ports

:USB

Flash Cards

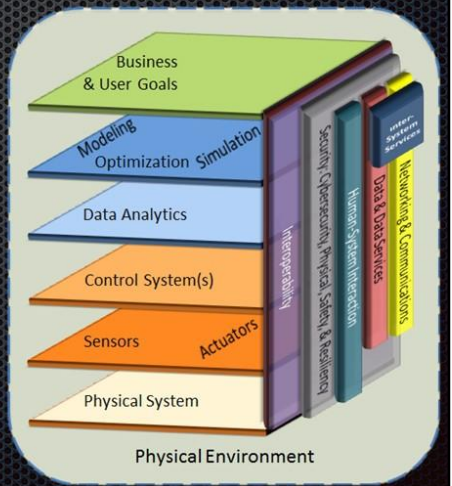




\title{
Security in the Printing
} System Firmware

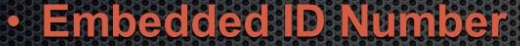 \\ - Access Manager \\ - Flle ID Token Authenticator \\ - Serial Number Generator \\ - Part ID Token Generator \\ - Activity Log Manager
}

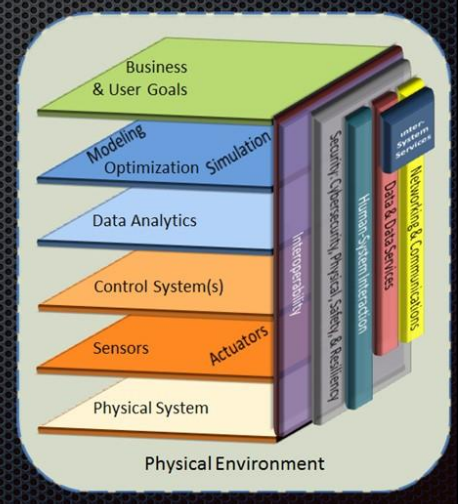

\section{Security in the Printing Control Software}

Incoming 3D file Package (JT ISO format*)

- authenticate (matchtile: D tokens)

- prepare for printing

- Watermark the print

control the printing process

- log each print

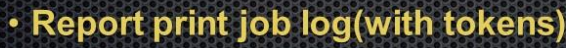

- Retain separate login print system firmware

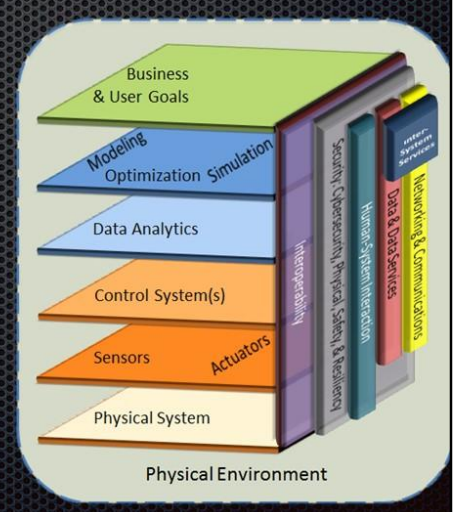




\section{Making Files Printable}

\section{File Prep:}

1. Analyse File \& Make Manifold

2. Input slice \& Position

3. Convert to Machine Language

Opportunity 5 or Ading Unique Info

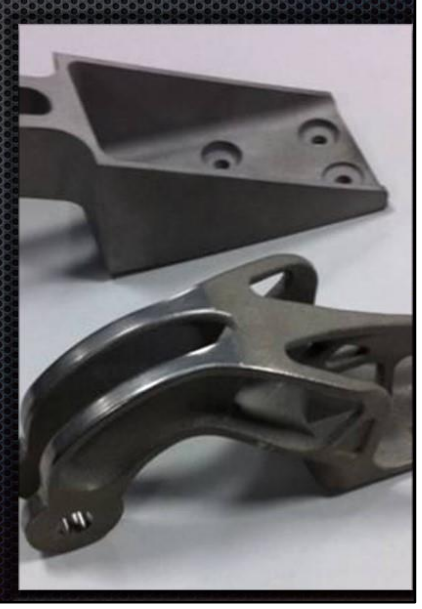

\section{File Description}

\section{Languages for 3D Printing}

STL Fles: Traditional format:

to be replaced by:

AMF: ISO ISOIASTM 52915

Standard specification for additive manufacturing file format (AMF)

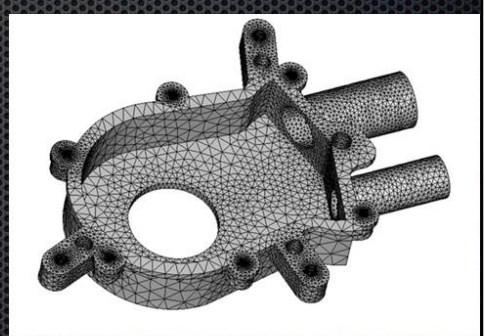




\section{AMF: ISO ISO/ASTM 52915}

Option for adoling watermarks

No Current Security Specified

Potentil for future Secility Specification
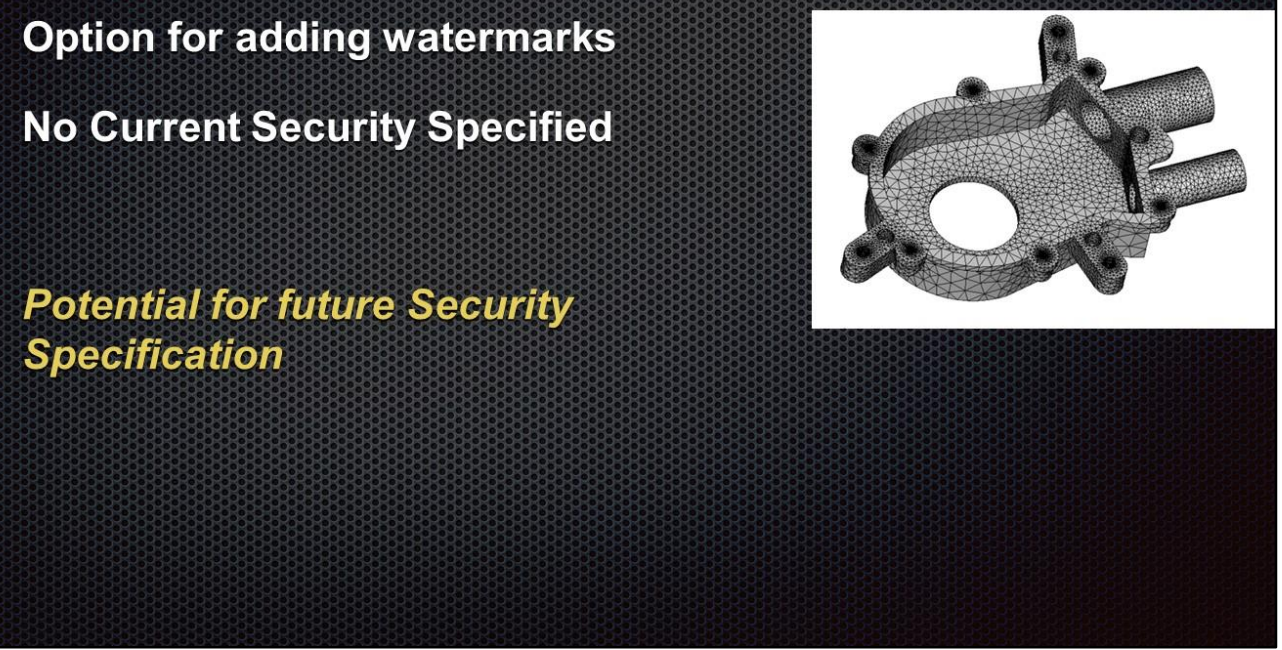

\section{AMF: ISO ISO/ASTM 52915}

\section{Potential for future Security S Secification:}

The <metadatas element:

Annex A1:

Allow for inclusion of copyright and other types of metadata

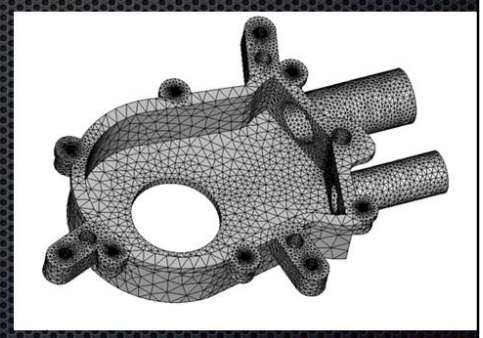




\section{Integrate With Current Factory Methods \& File Formats}

JT Open Format (ISO ISO 14306)

1. 3D file package

2. carry security tokens

3. transmit across network

4. deliver to printing system

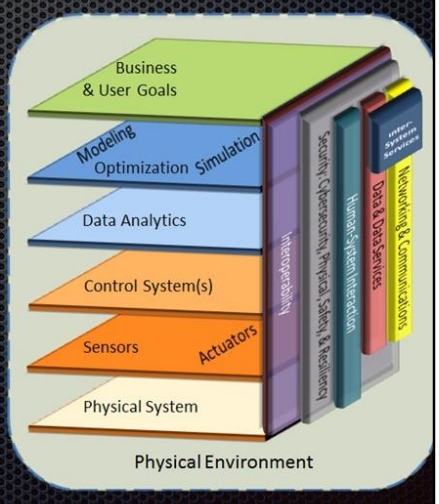

\section{A Simple, Cost Effective,}

\section{Scalable Approach}

1. Utilize JT ISO \& AMF:ISO File Formats

2. Integate with Printer Mgt: Software

3. Provide A Token Based Automatic

Authentication Process

Secure Embedded Authenticationin Firmware

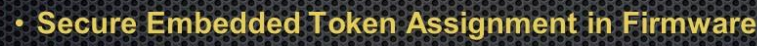

4. Implement a Redundant Print Job log System

stored in printermemory (locked)

publish to authorized recipients on network (JT)

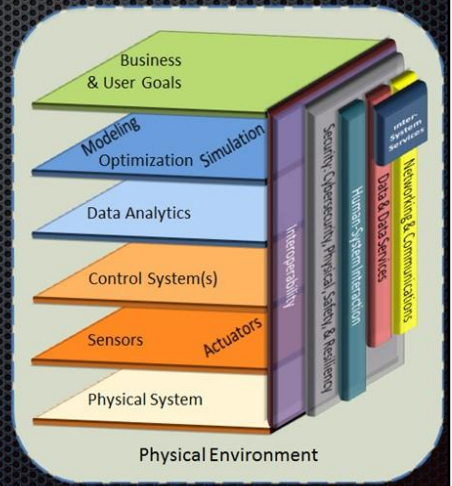




\section{A Simple, Cost Effective, Scalable Approach}

\section{Printer Management Software:}

- Compare tokens in firmware with hooning

Convert JT to AMF 1 SO format

- Prepare print job for printing

- Generate unique part ID from printer firmware - create unique waterimaik for each part

- slice and generate Gicode

Save the print job info to Print Log

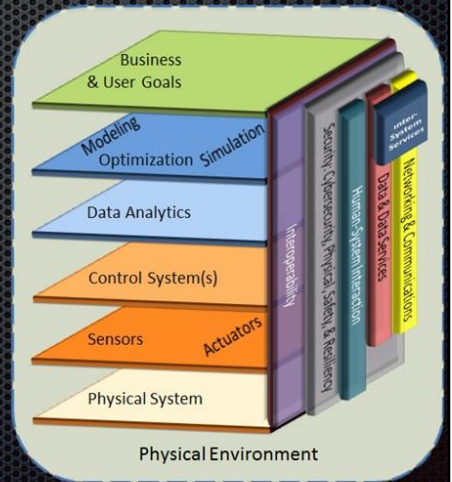

\section{The Print Job Log}

Includes:

-: print job numberio

- prints by serial number

:a: Prints by iD token:

$\because$ printer location \& 10

:

: ${ }_{\text {If }}$ additional data (optional)

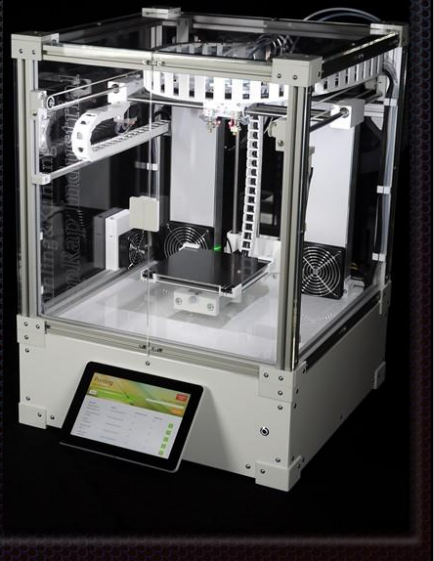




\section{A Simple, Cost Effective, Scalable Approach}

\section{Redundant, Secure Records}

- Save the print job info to Print

- Generate unique loo token for the Print

- Convert to JT format file

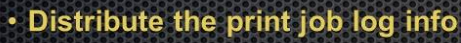

to printer memory (protected)

: to requesting parties on the network

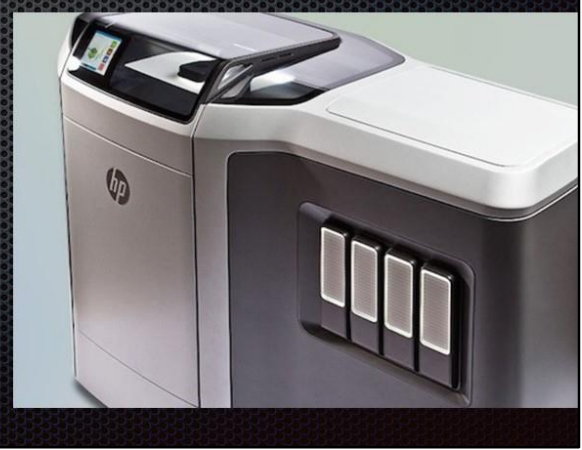

\section{Leveraging Manufacturing Systems}

Systems for:

- Revision Control

- Quality Control

- Customer Matching

- Product Tracking

- Quality Assurance

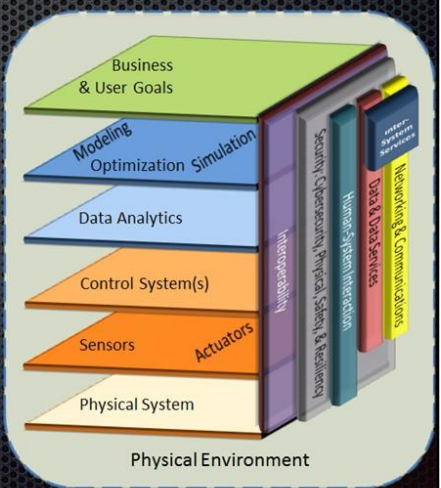




\section{Materials Can Also Contain Security Components}

- Chemical Tagging

- Physical Tagging

- Physical Watermarking

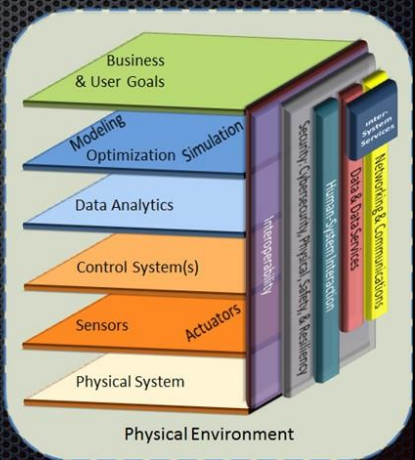

\section{Making Printable Parts}

Quality without Security =: 


\section{Key Points}

1. Security Starts at the Printer

2. Leverage ISO Standards

3. Plan for Desktop to Large Printers

4. Security Does NOT have to be Expensive

5. Start NOWI

\section{Thank You!}

For More information:

Avante Technology, LC. 


\section{Presentation by Claire Vishik:}

\section{NIST Cyber Physical Systems Public Working Group}

Korean Ministry of Science, Technology, ICT and Future Planning (MSIP)

November 18, 2014

Victoria Yan Pillitteri

Victoria.yan@nist.gov
NLT

National Institute of

Standards and Technology

U.S. Department of Commerce

About CPS PWG 


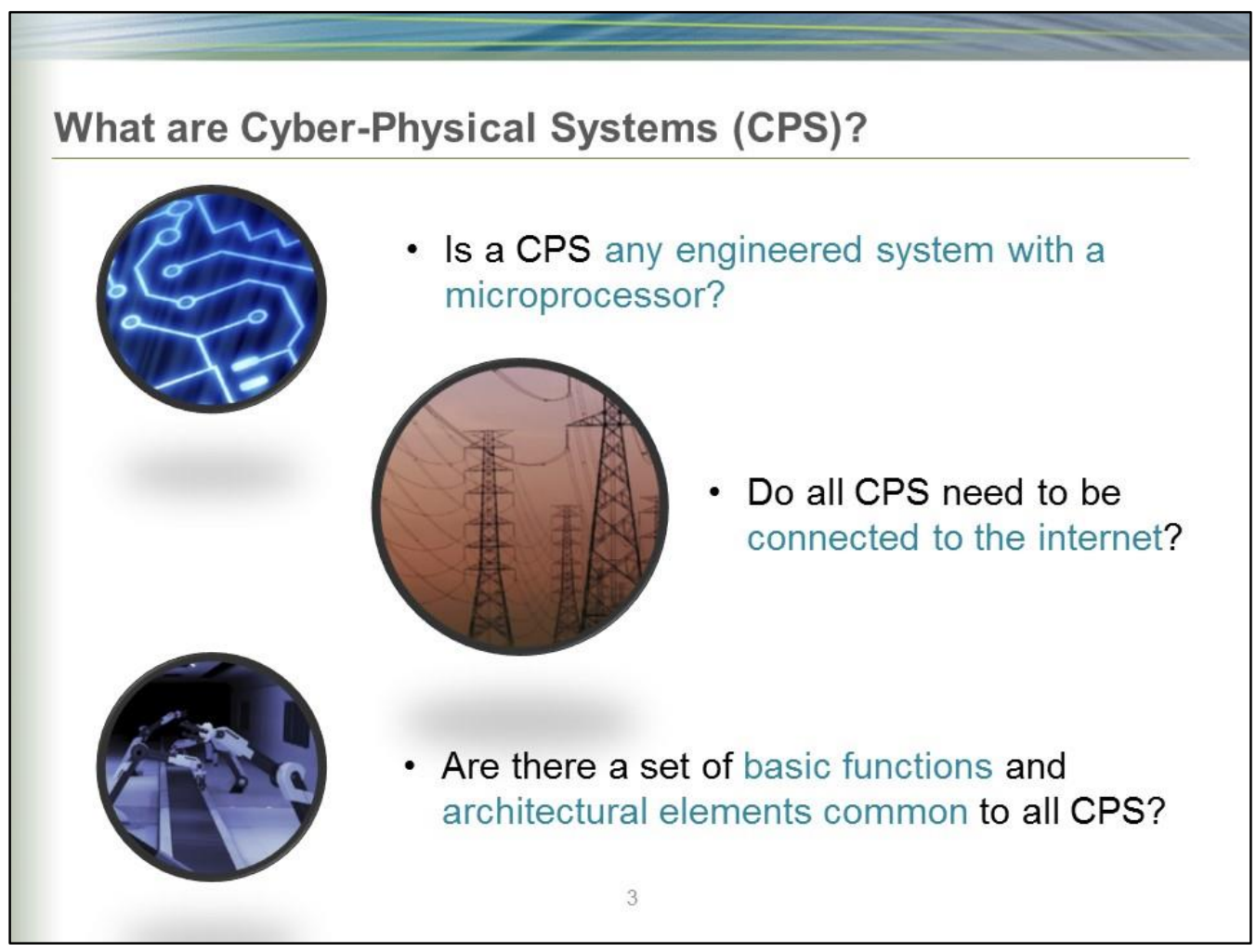

CPS - Notional Definition

- Integrated, hybrid networks of cyber and engineered physical elements

- Co-designed and co-engineered to create adaptive and predictive systems

- Respond in real time to enhance performance* ${ }^{*}$ Key metrics include: efficiency and sustainability, agility and flexibility, reliability and
resilience, safety and security 


\section{Why is NIST convening the Public Working Group (PWG)?}

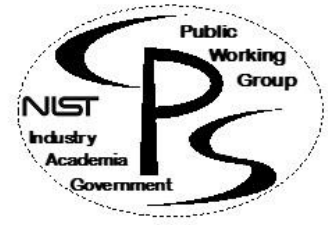

- Accelerate progressing CPS across all sectors and domains.

- Currently lack a unified technical foundation for broad collaboration.

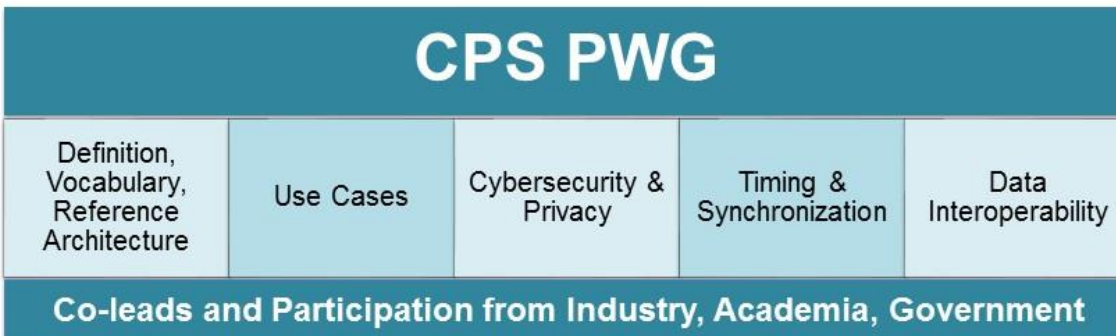

\section{CPS PWG Goal and Deliverables}

Winter 2014

Initial Report

from each

subgroup

- Definition, Vocabulary, Reference Architecture

- Use Cases

- Cybersecurity \& Privacy

- Timing \& Synchronization

- Data Interoperability
Spring 2015

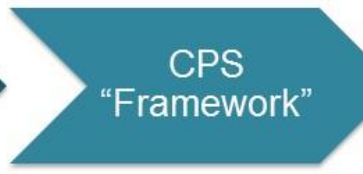

- Integrate subgroup reports

- Publish as white paper on www.cpspwg.org
Summer 2015

\section{CPS}

Technology

Roadmap

- Identify opportunities for a coordinated effort on key technical challenges from each subgroup area and across CPS 


\begin{tabular}{l} 
CPS Cybersecurity and Privacy Subgroup \\
\hline Goal/Objectives: \\
- Identify the unique challenges, properties and opportunities for CPS \\
- Develop appropriate cybersecurity objectives (e.g., confidentiality, \\
integrity, and availability) for CPS \\
Contribute to the overall reference architecture for CPS to ensure \\
that cybersecurity is included and addressed \\
Identify security and privacy requirements for the components of the \\
reference architecture.
\end{tabular}

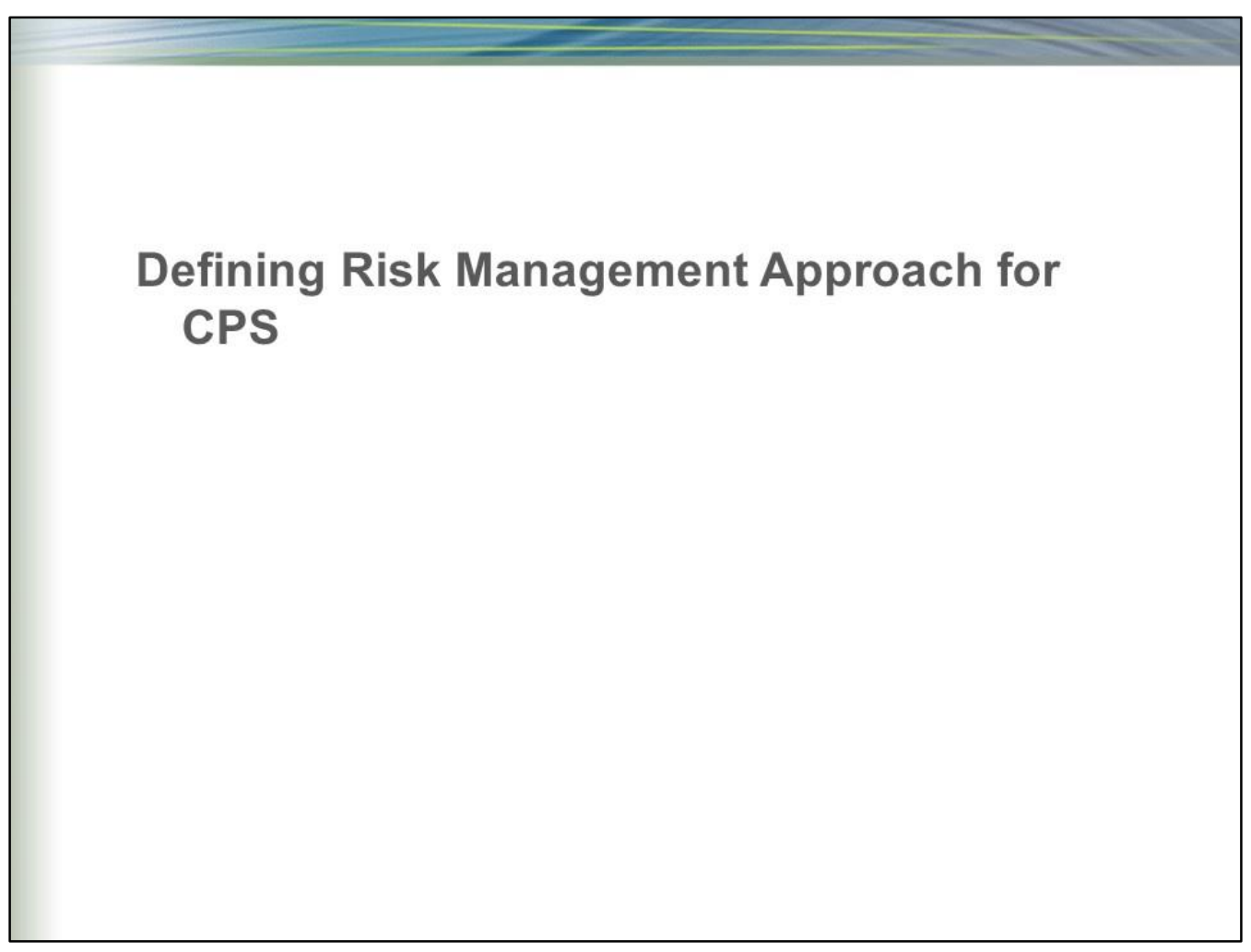




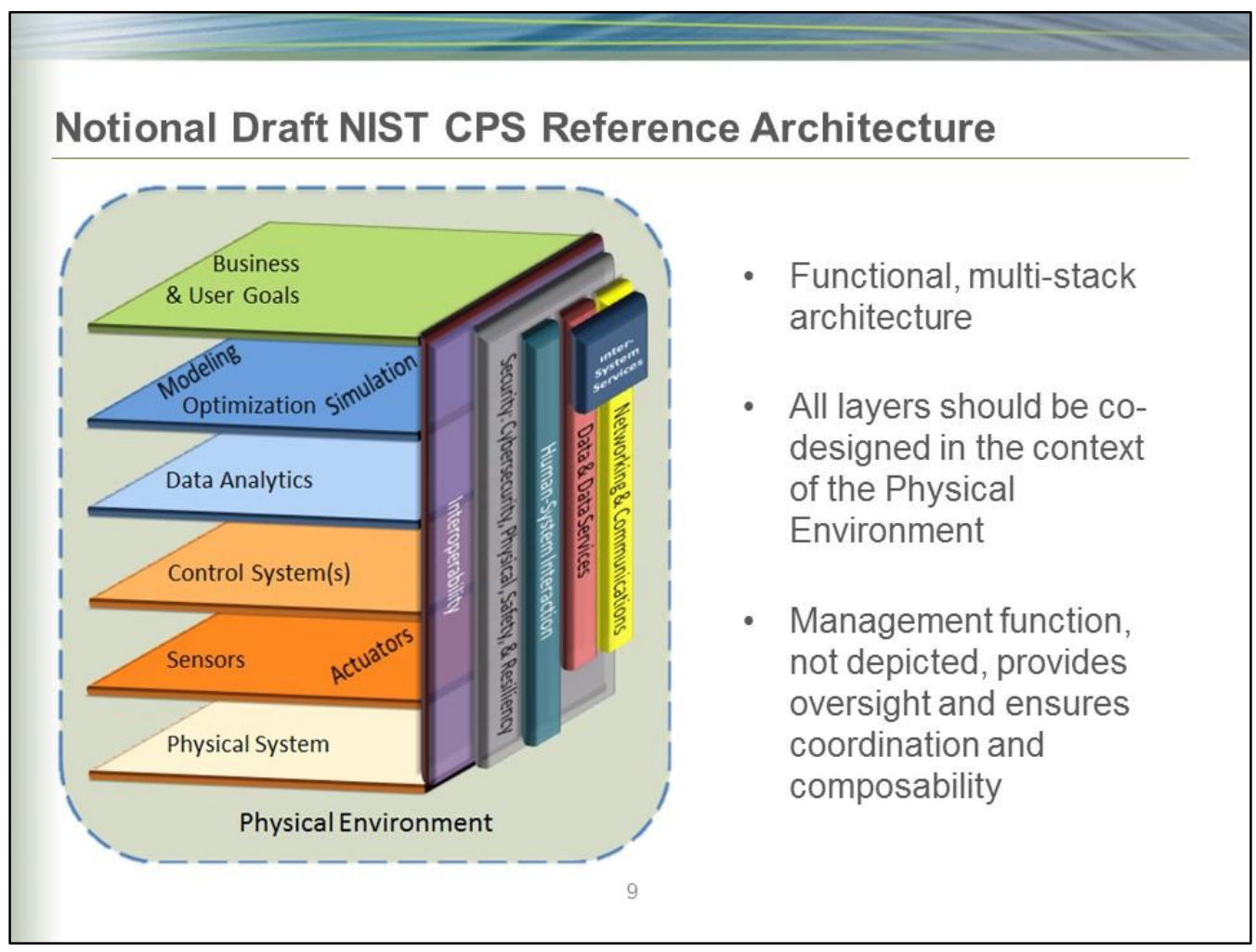

Some Properties of CPS: very early work

- Need for resilience, reliability, and safety in addition to security \& privacy

- Wide range of operational contexts

- Lifecycle challenges, including differing lifespans

- "Systems of systems" resulting in inherent complexity

- Impact on physical world

- Time sensitive

- Resource constrained elements

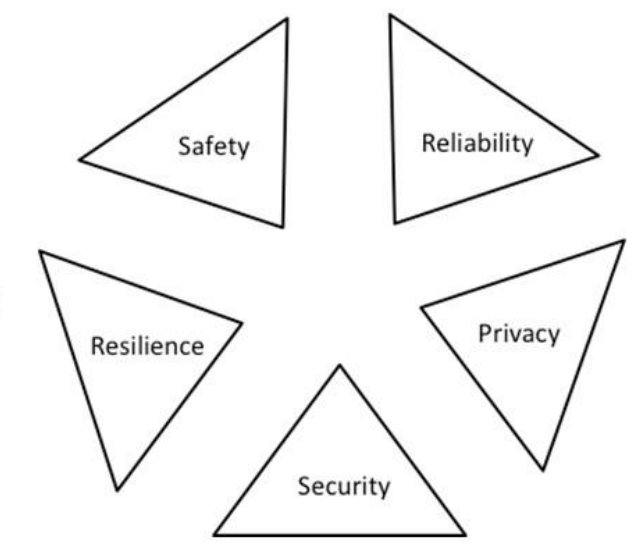

- "Always on" requirements 

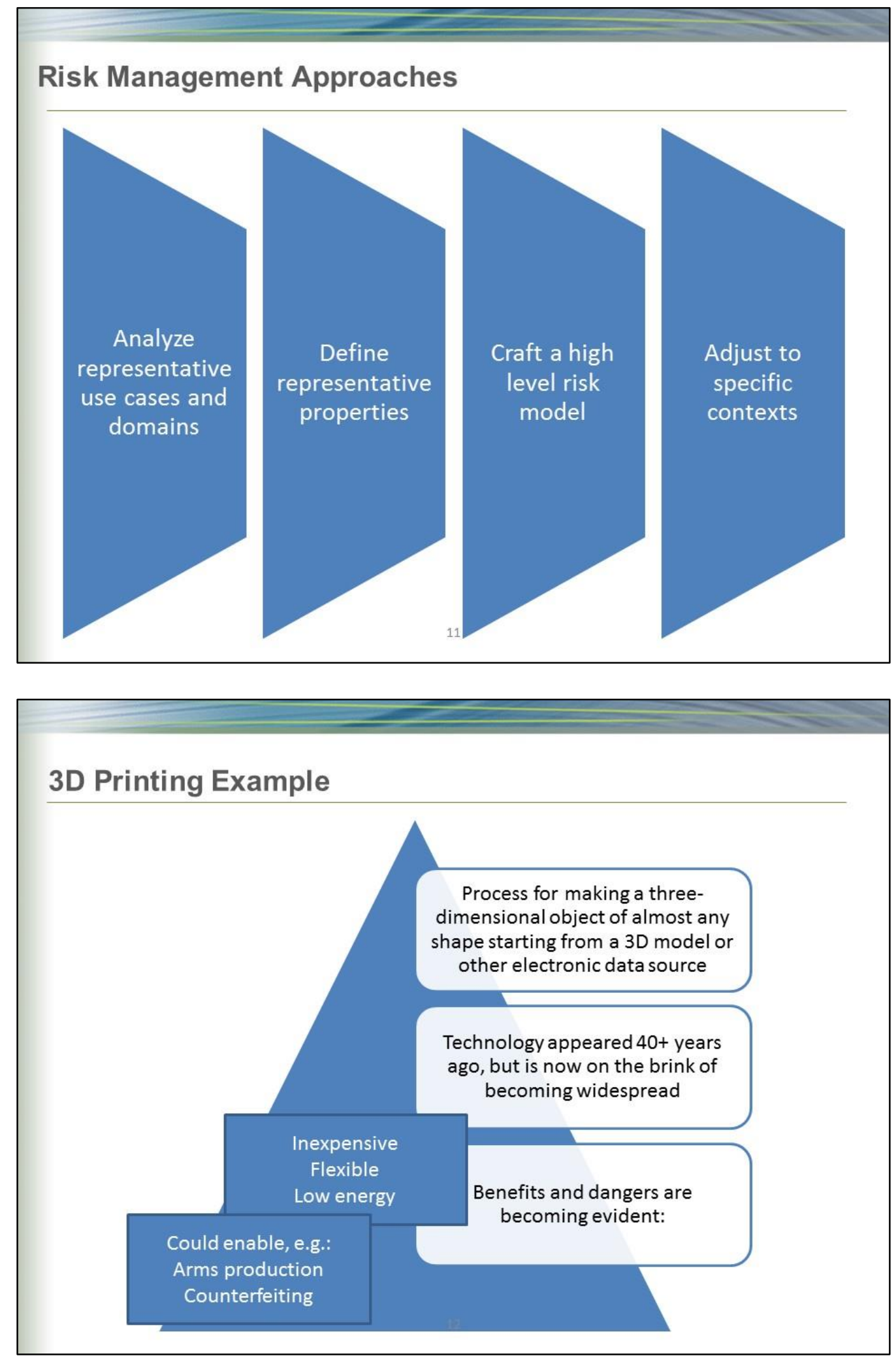

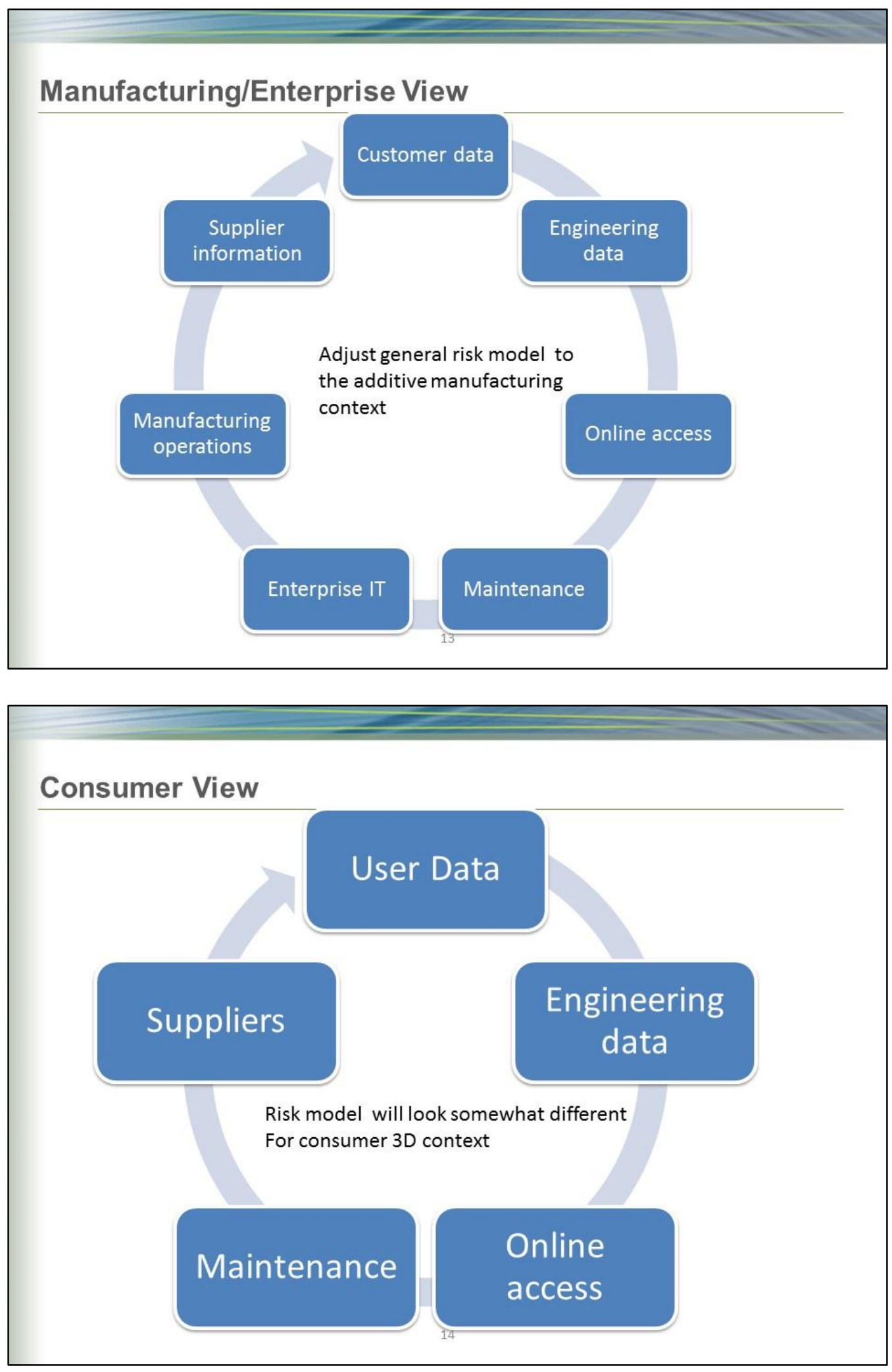

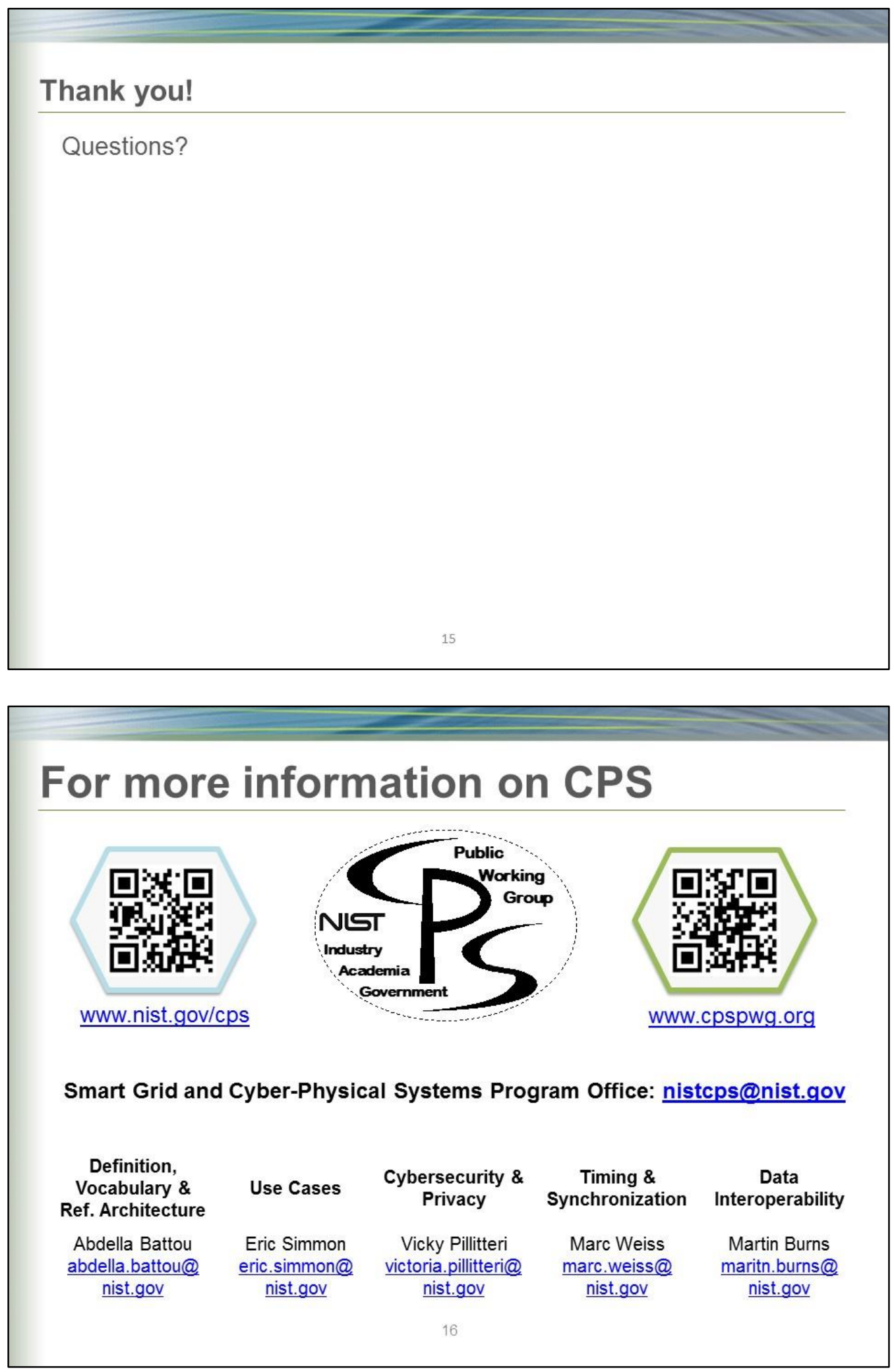


\section{Presentation by Andre Wegner:}
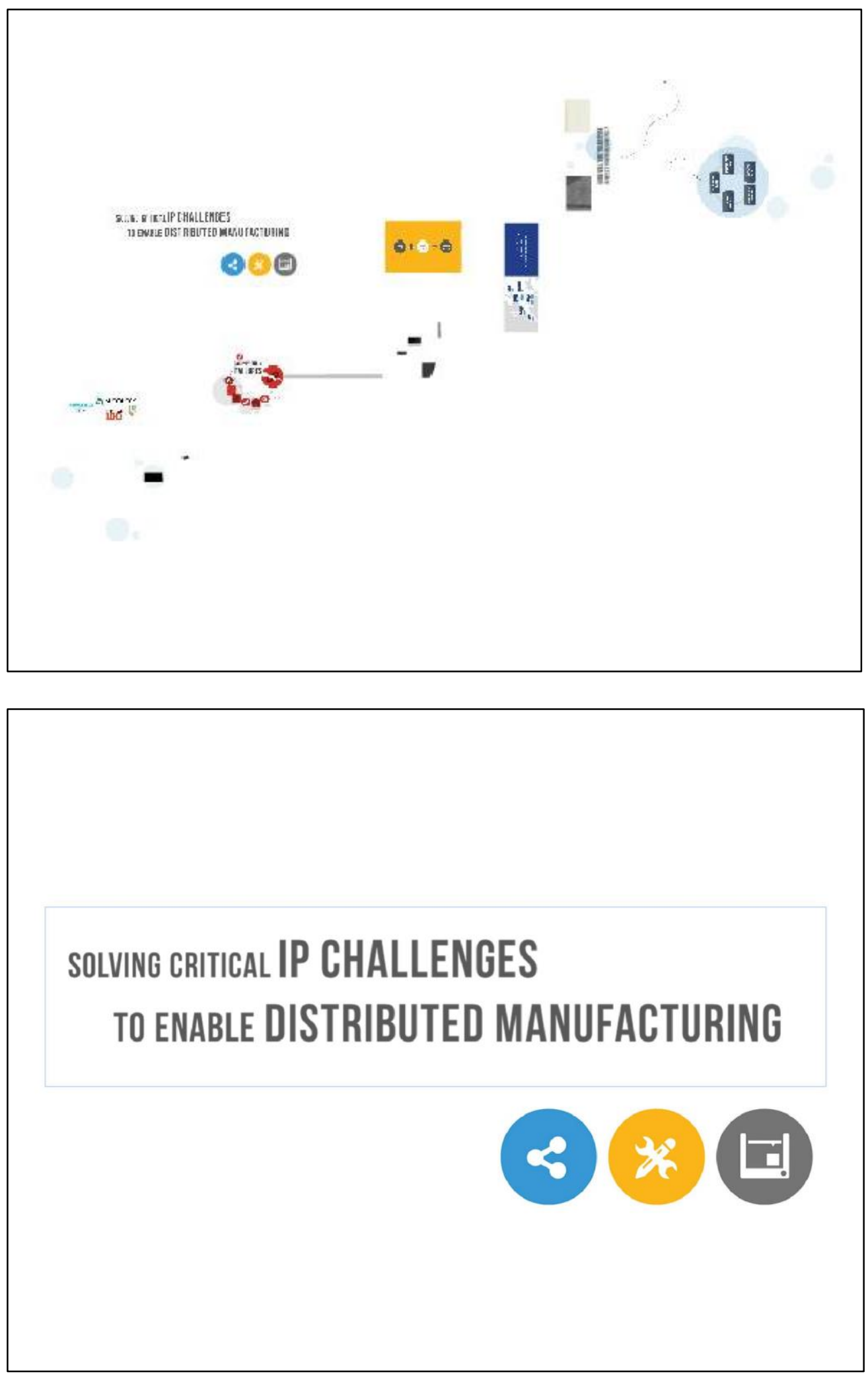

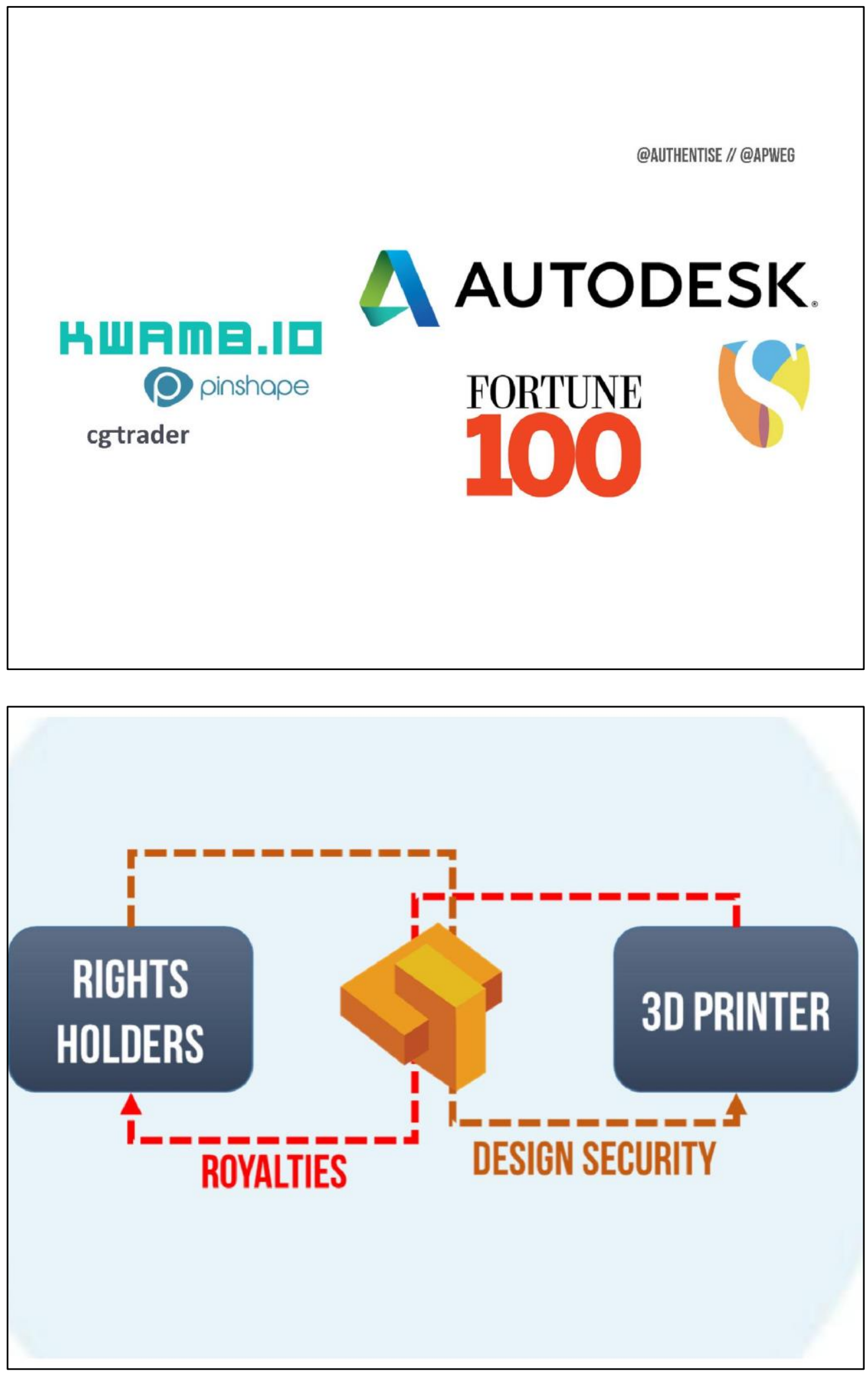

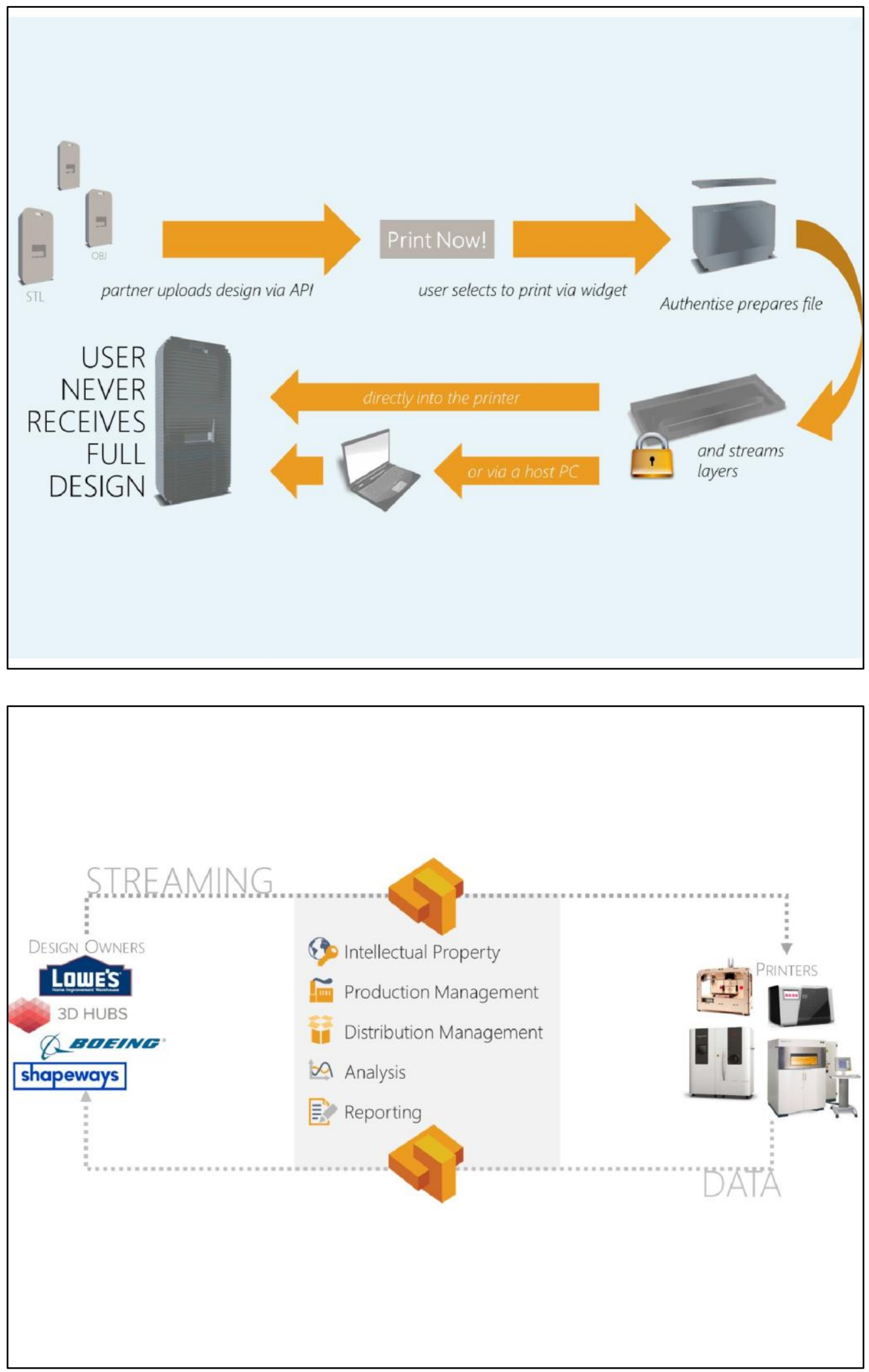

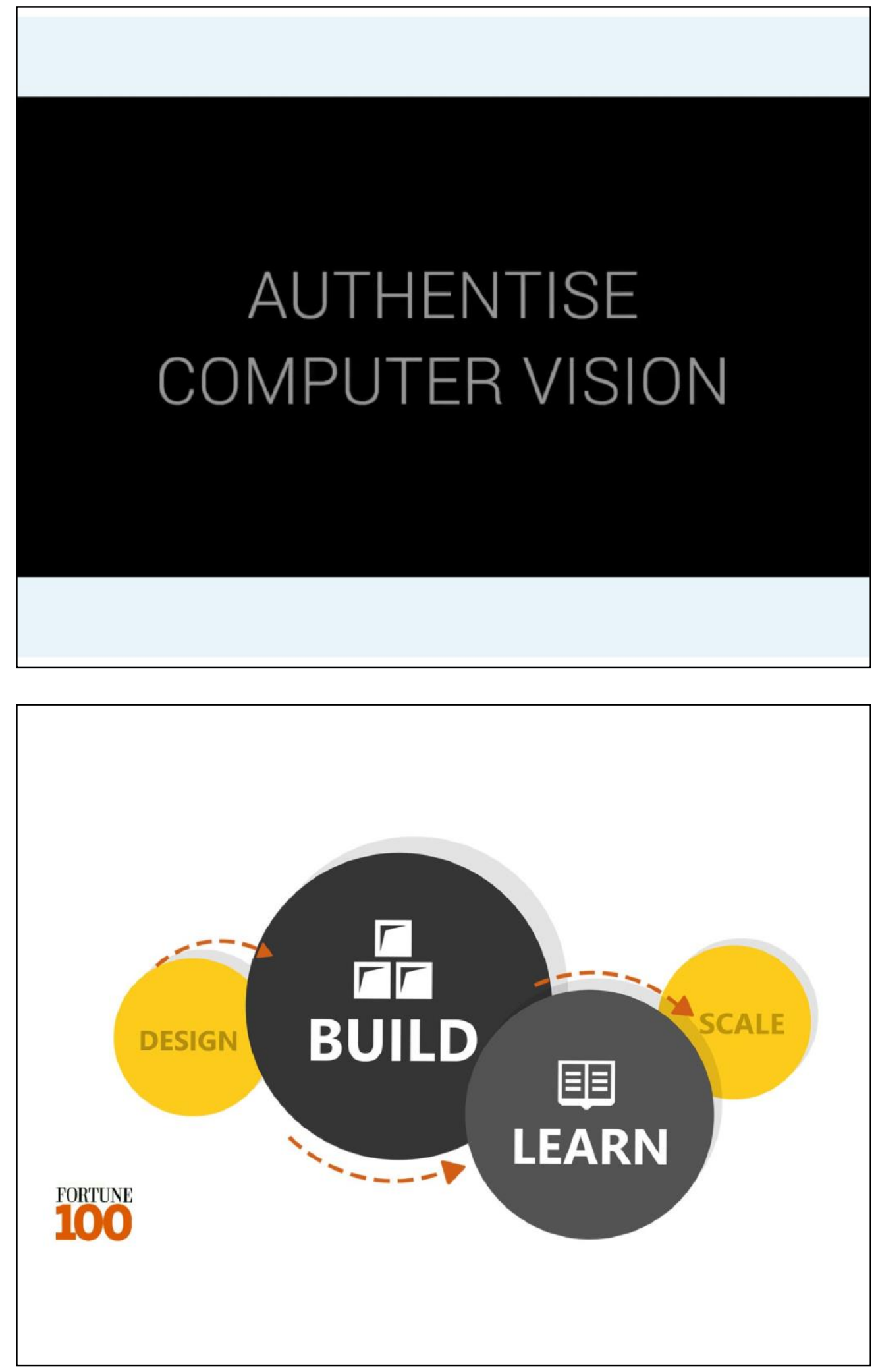

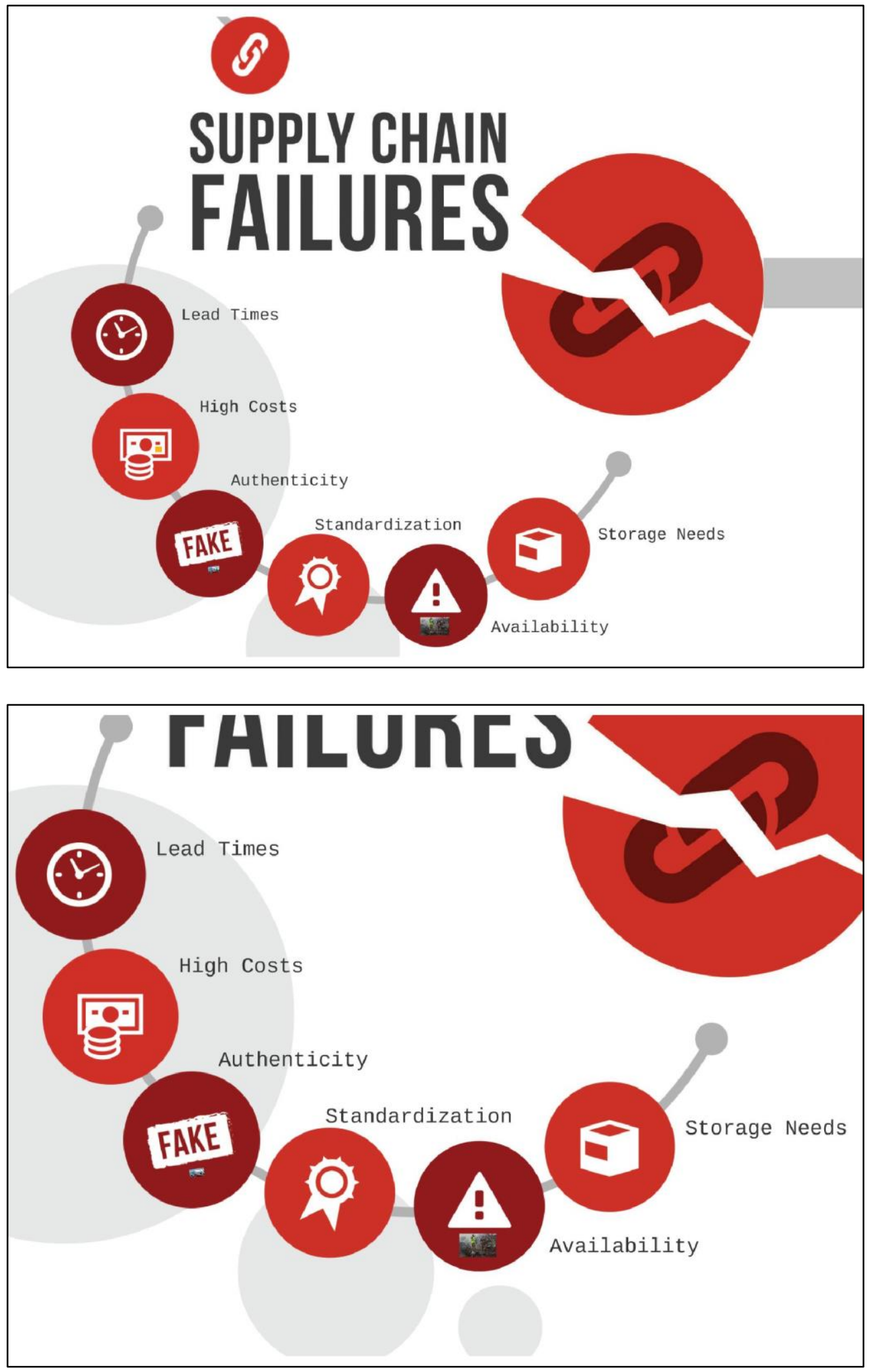

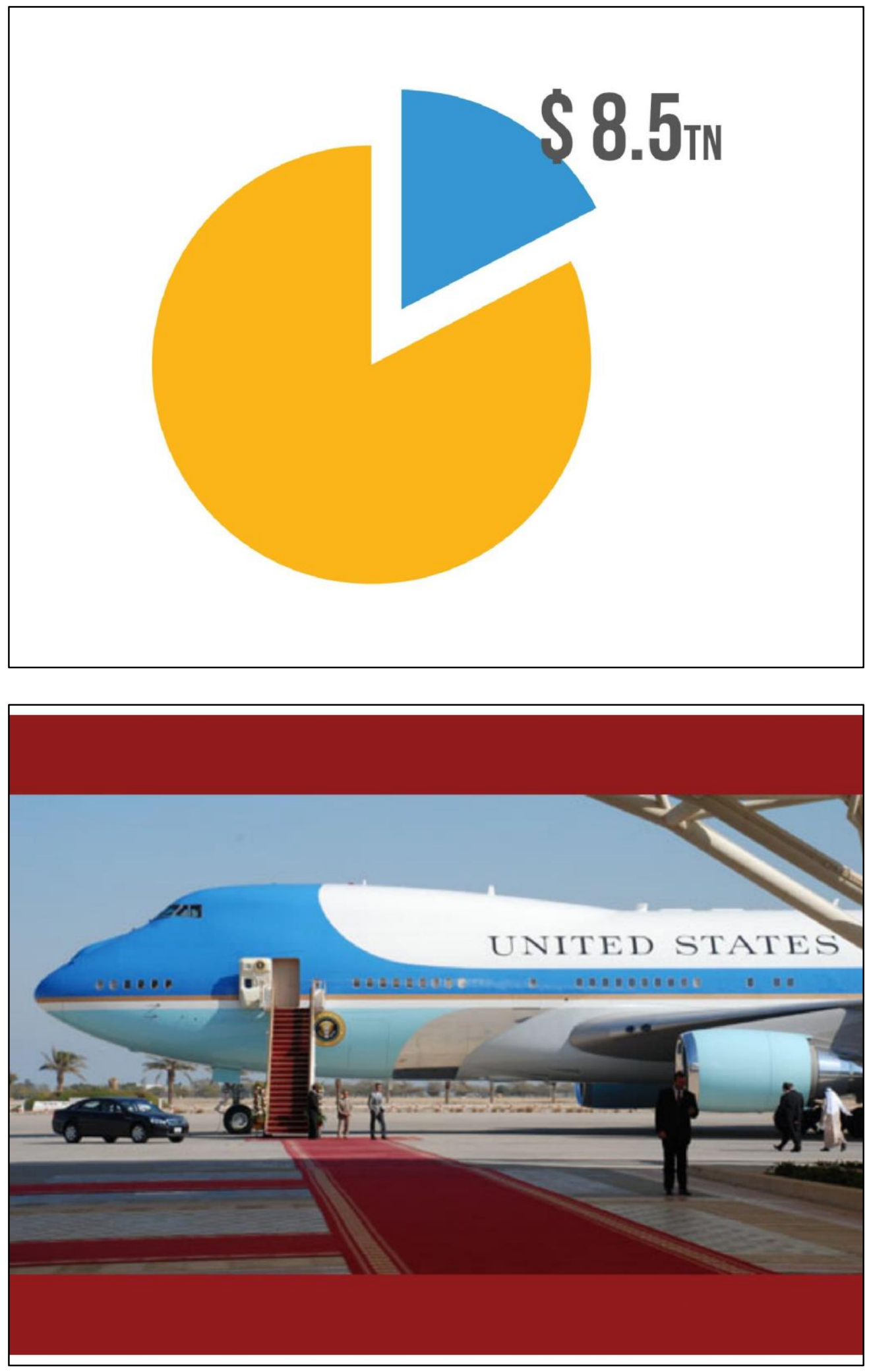

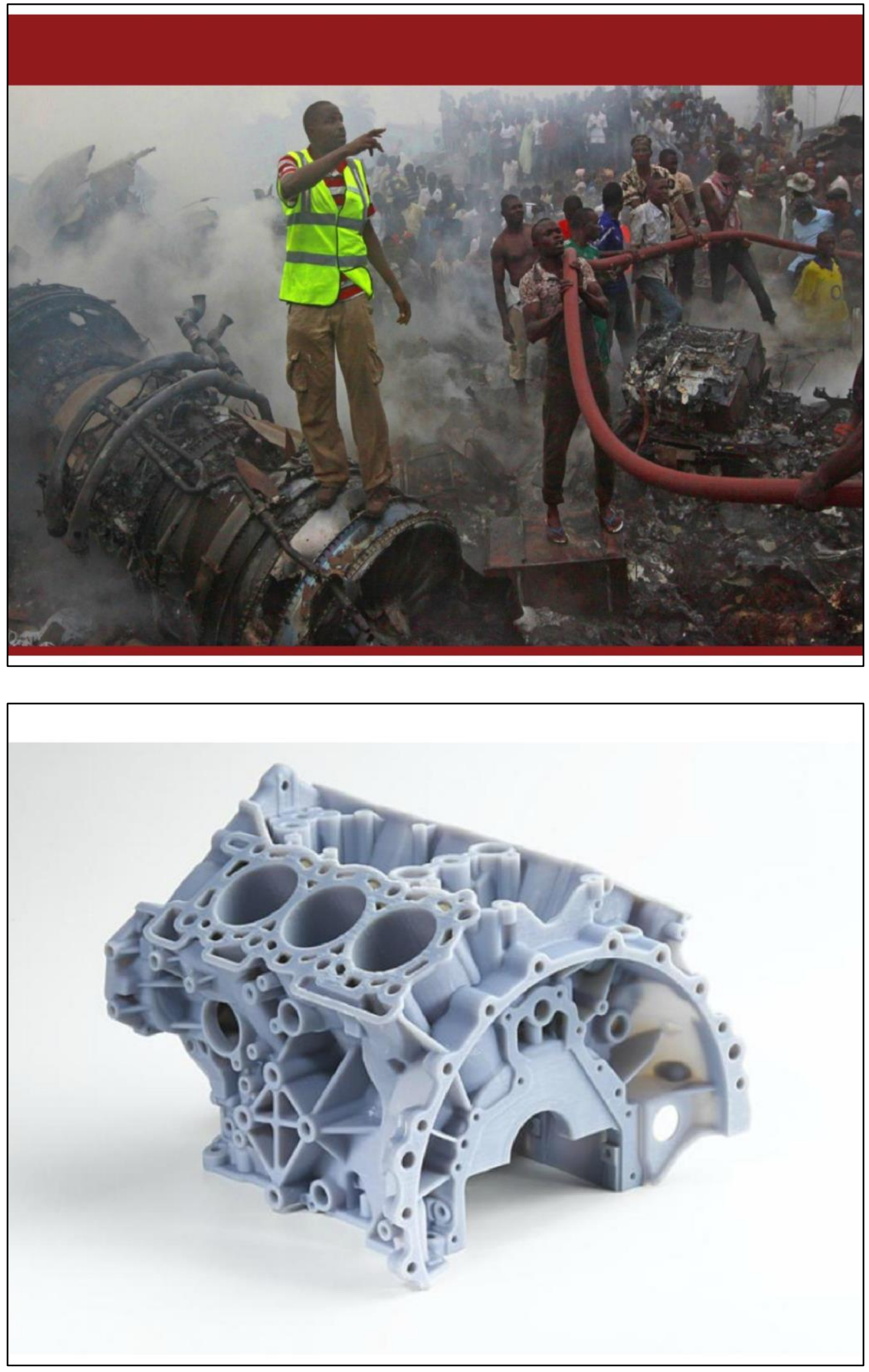

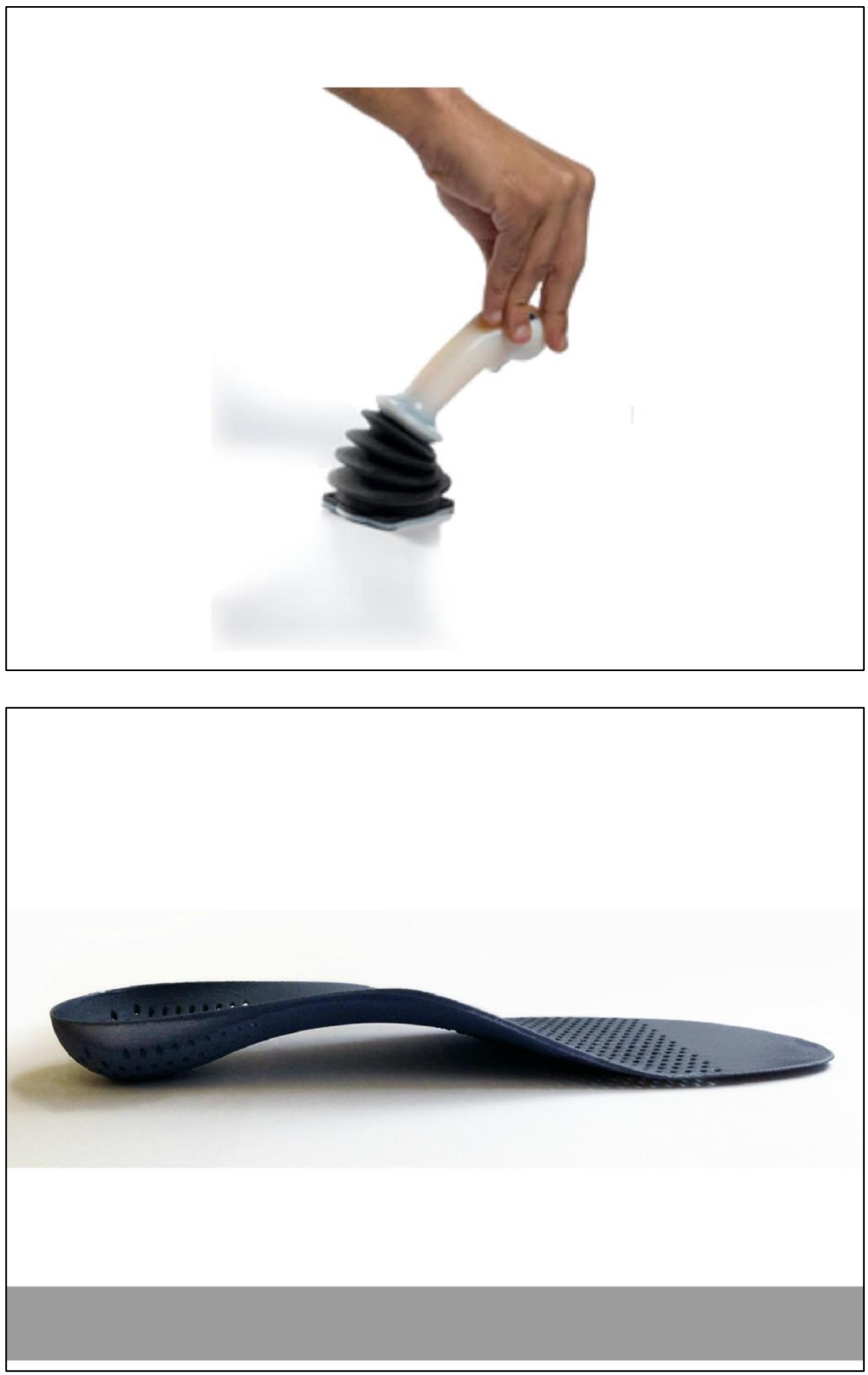

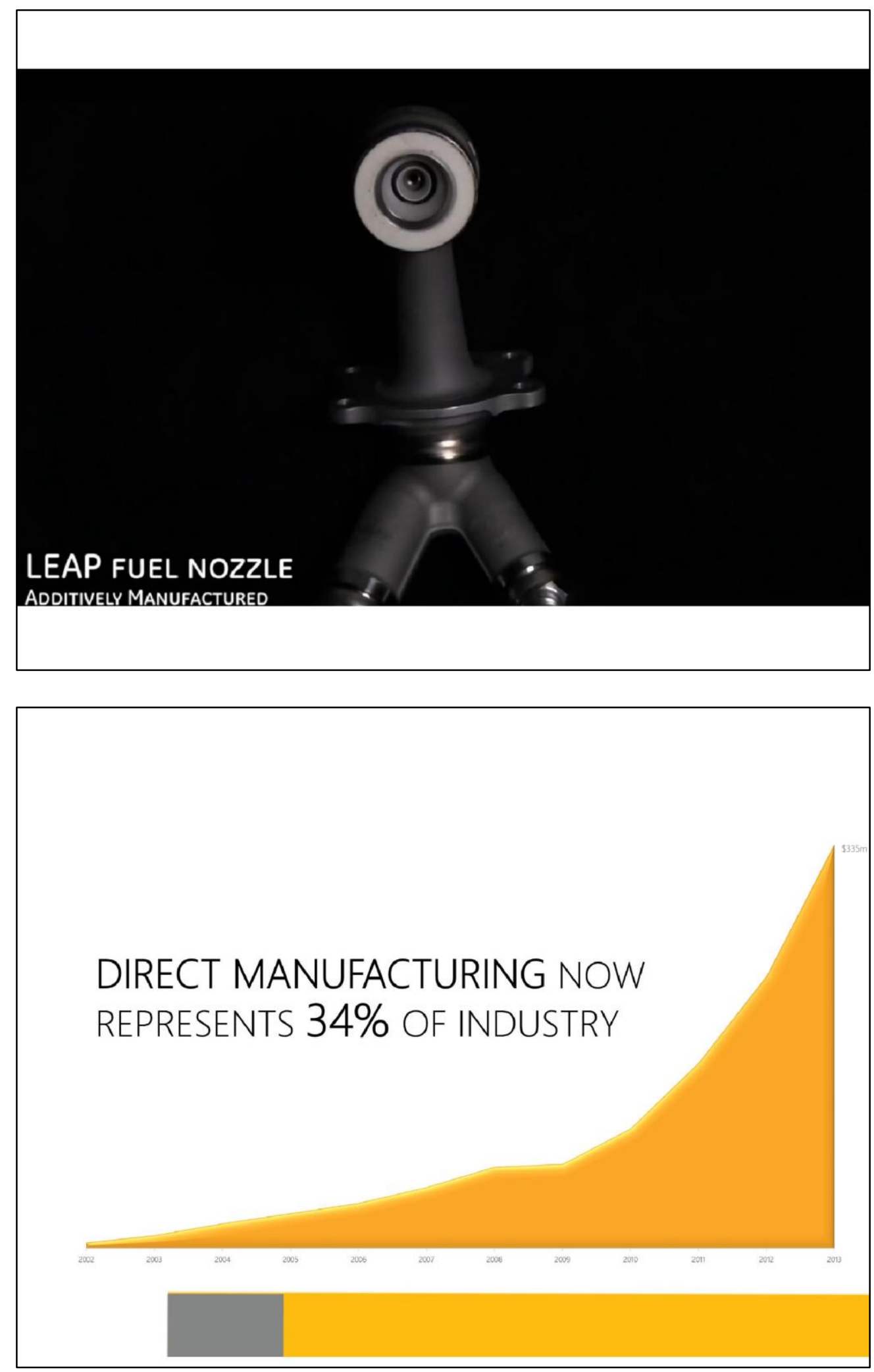

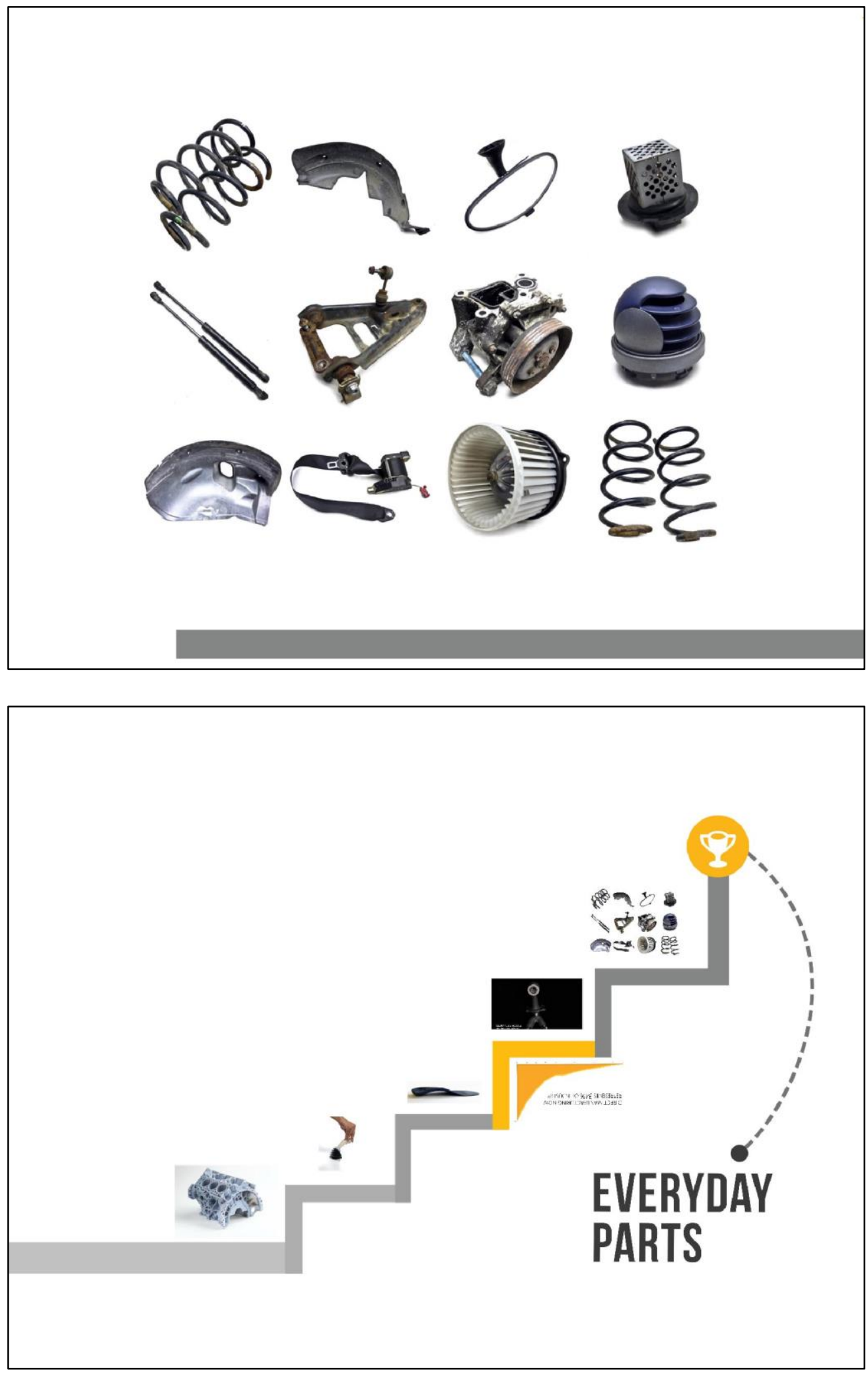

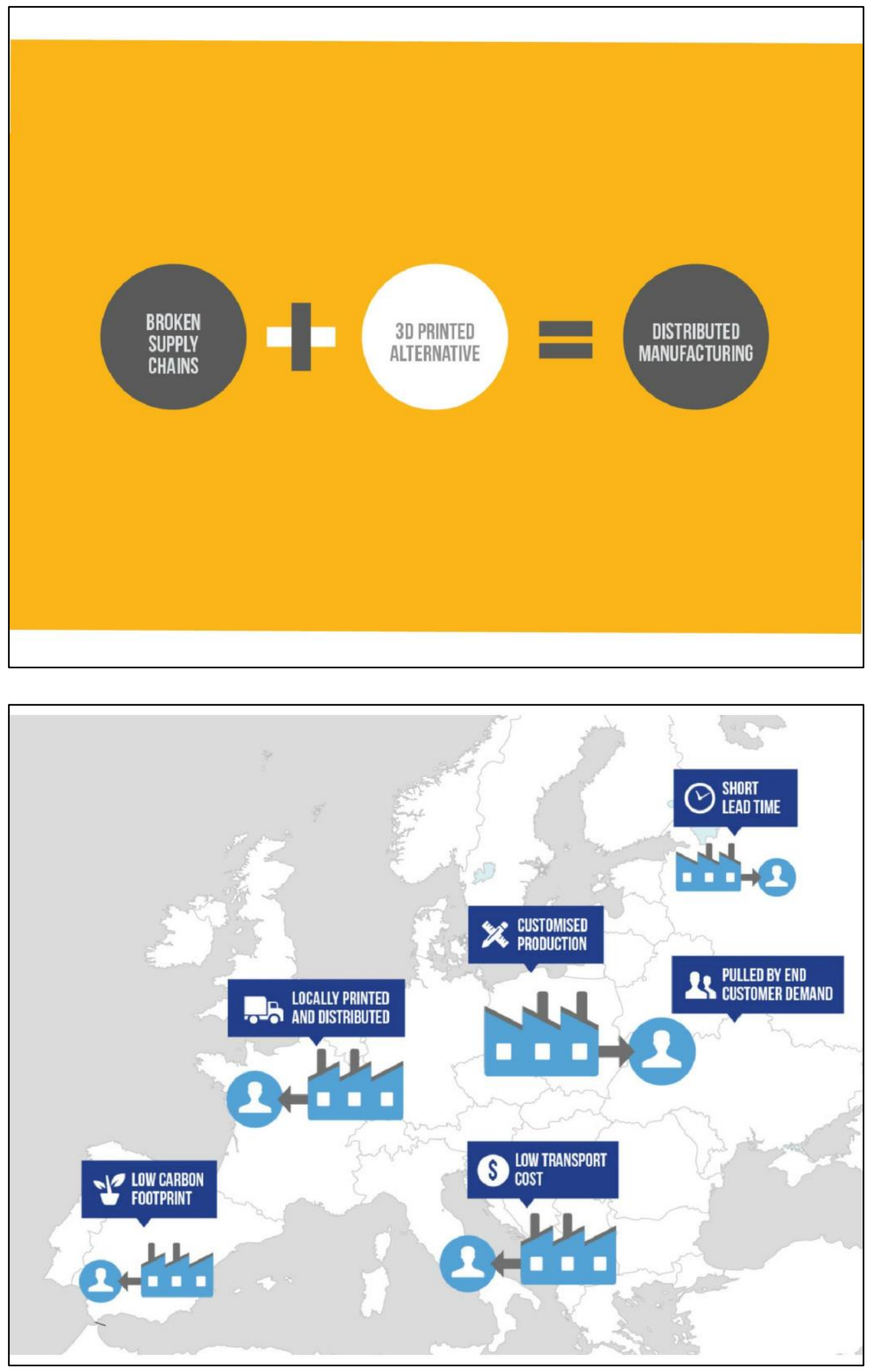

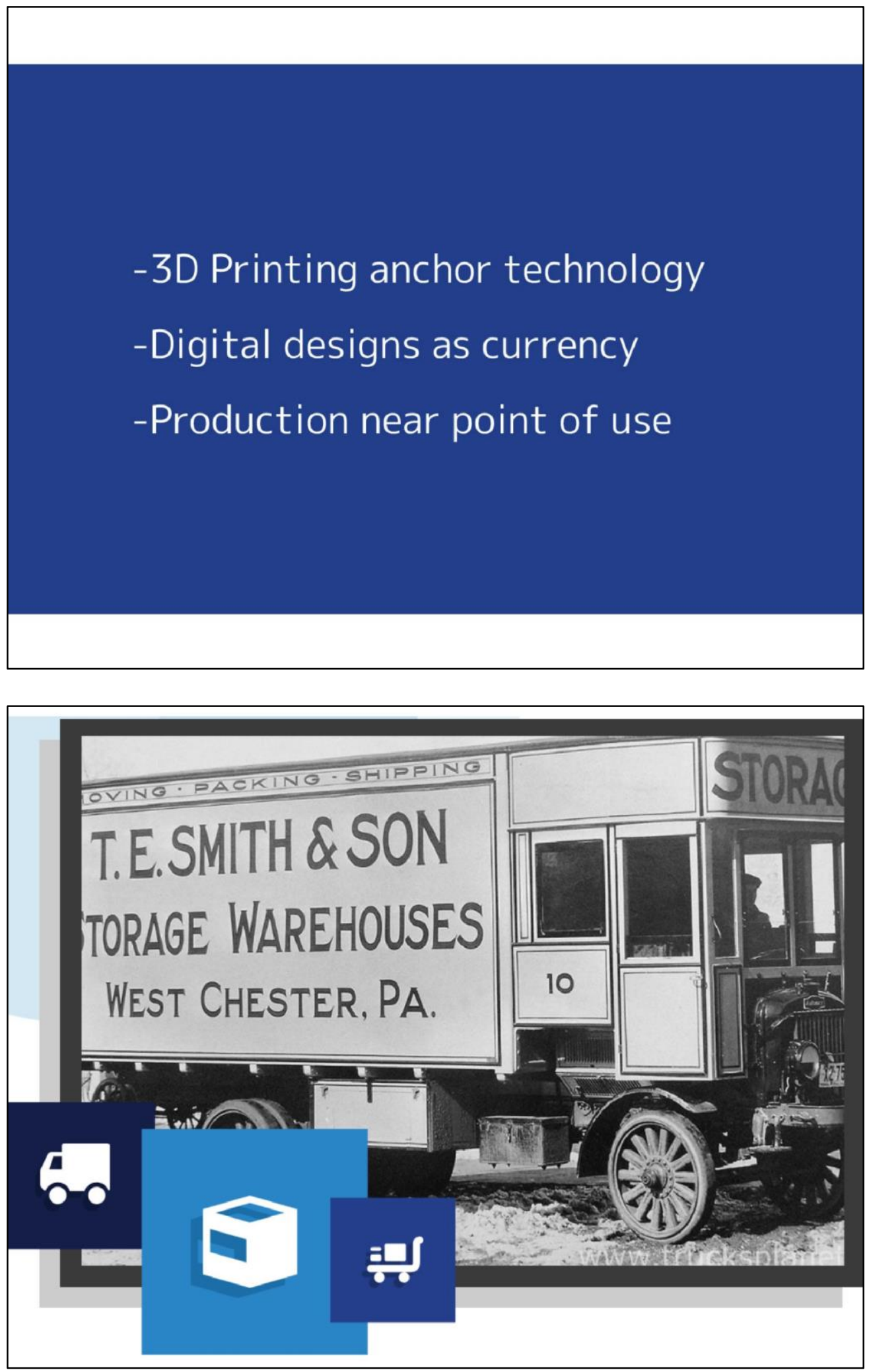

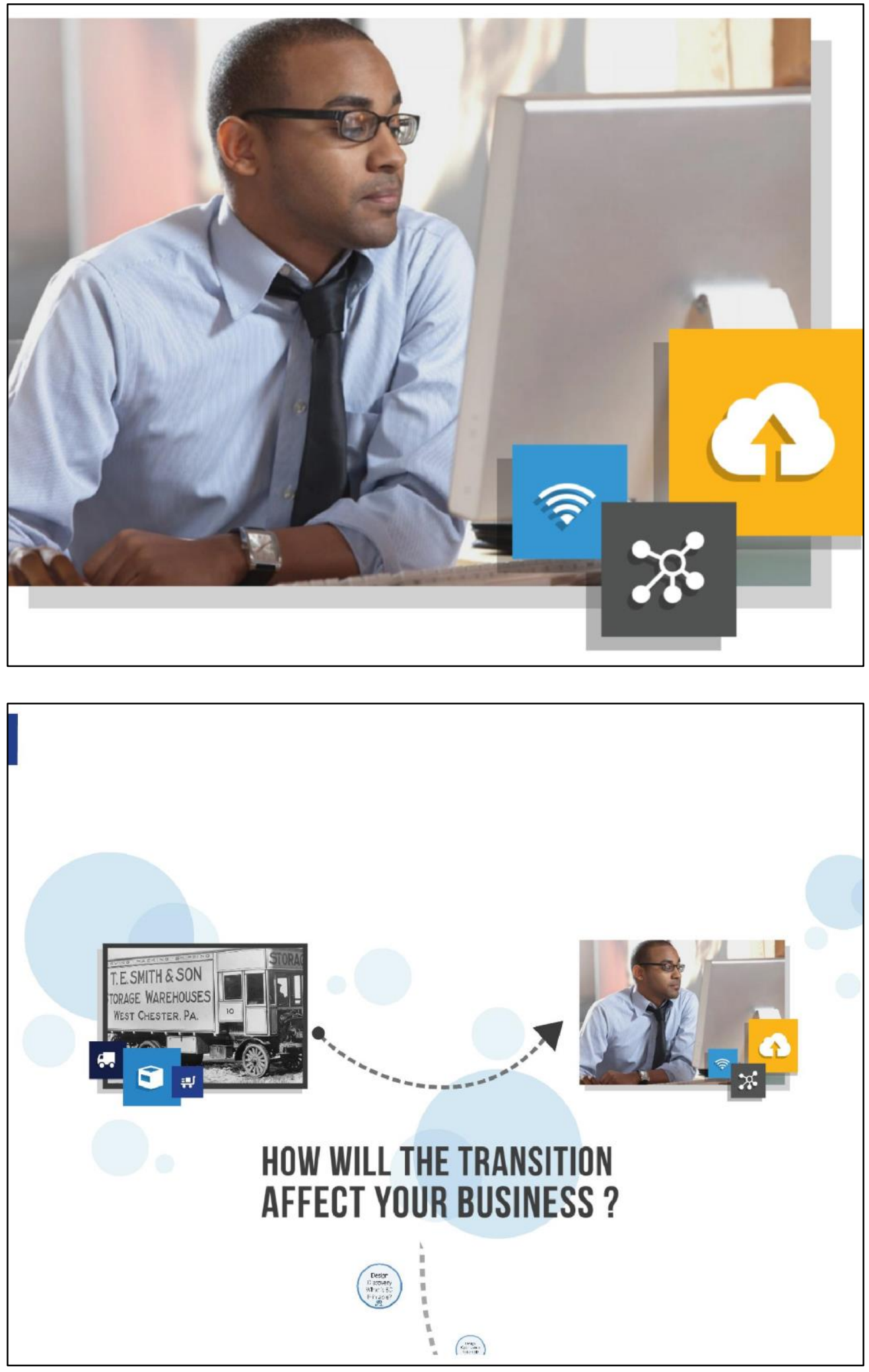

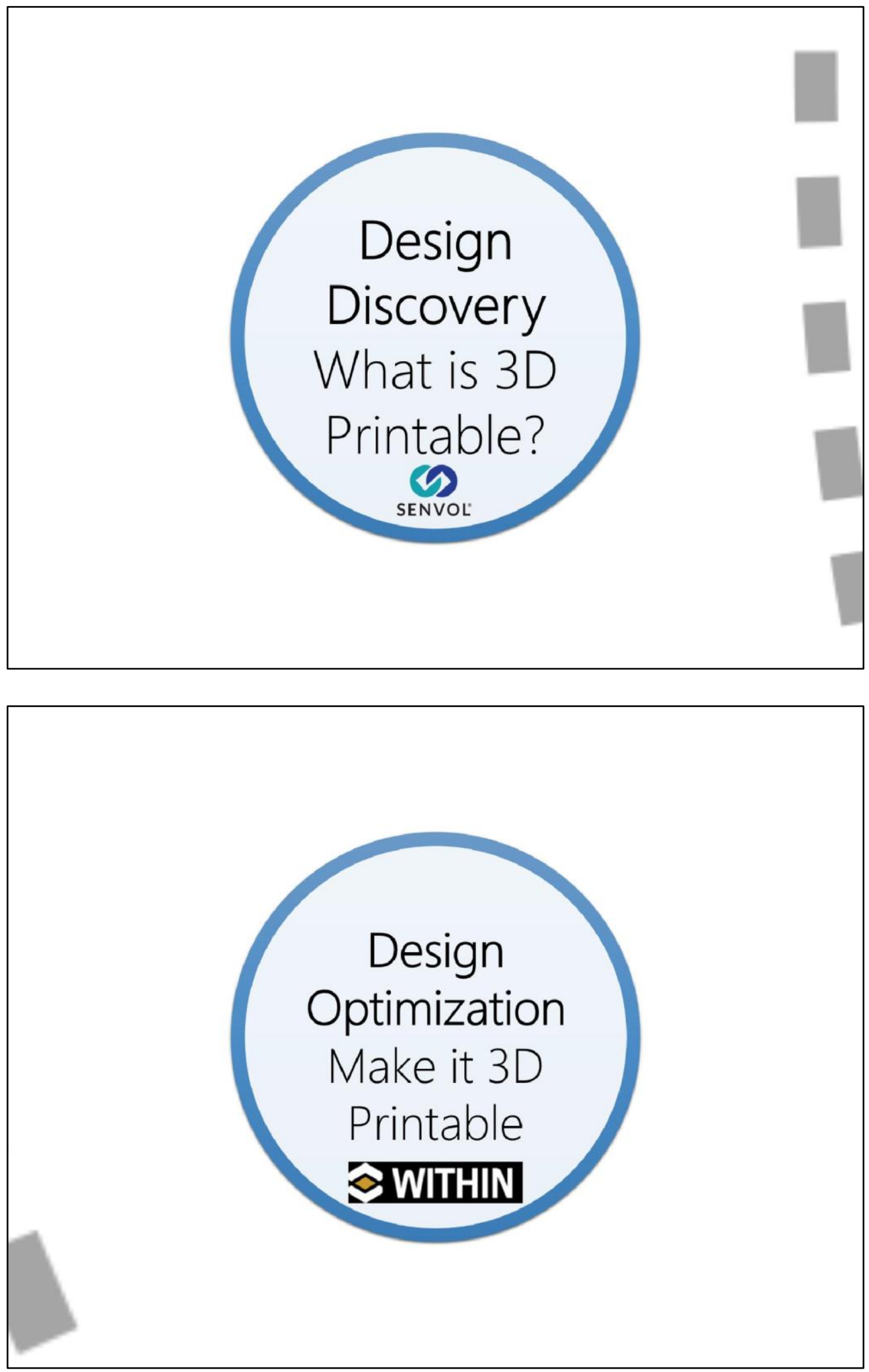

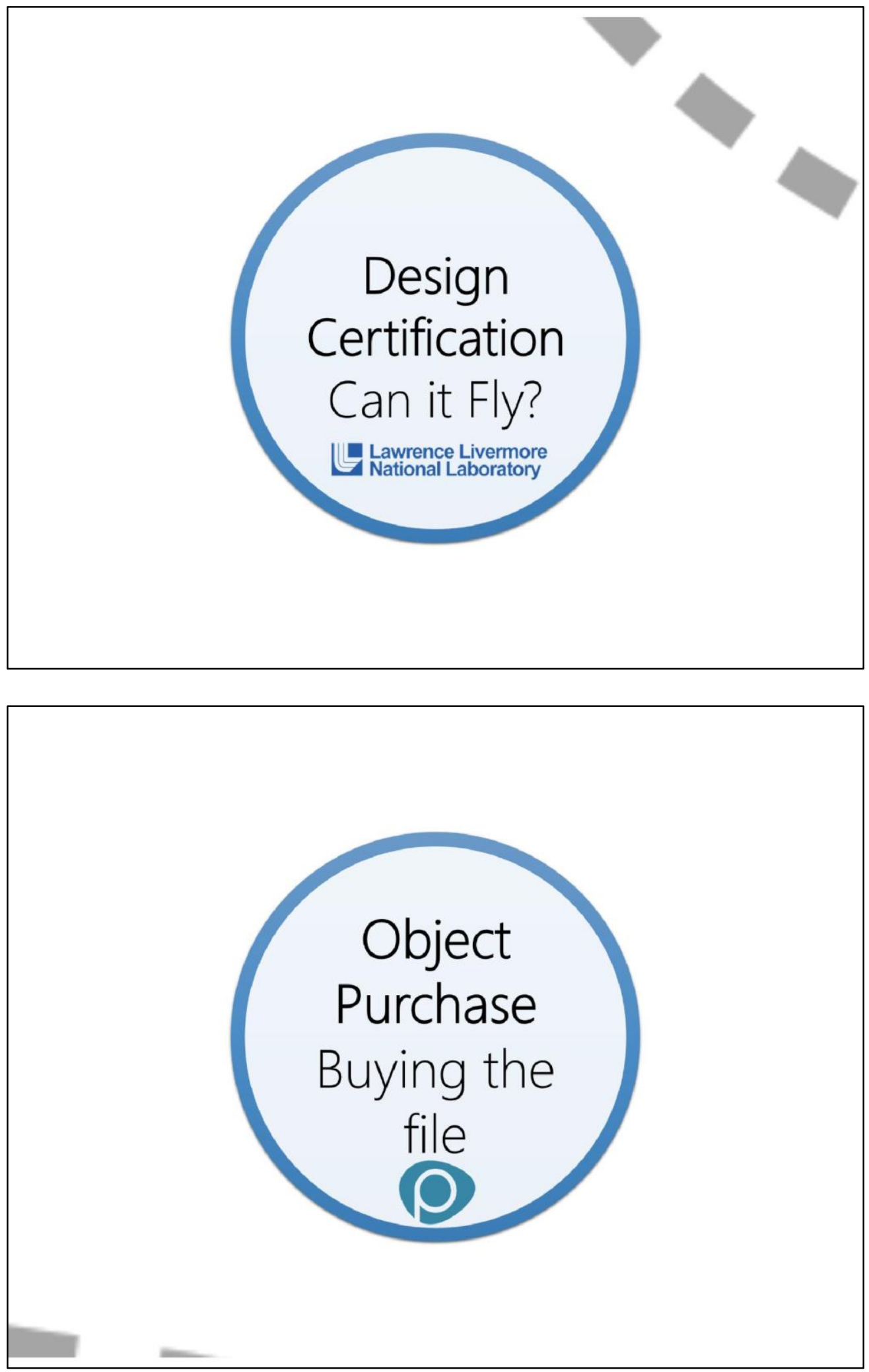

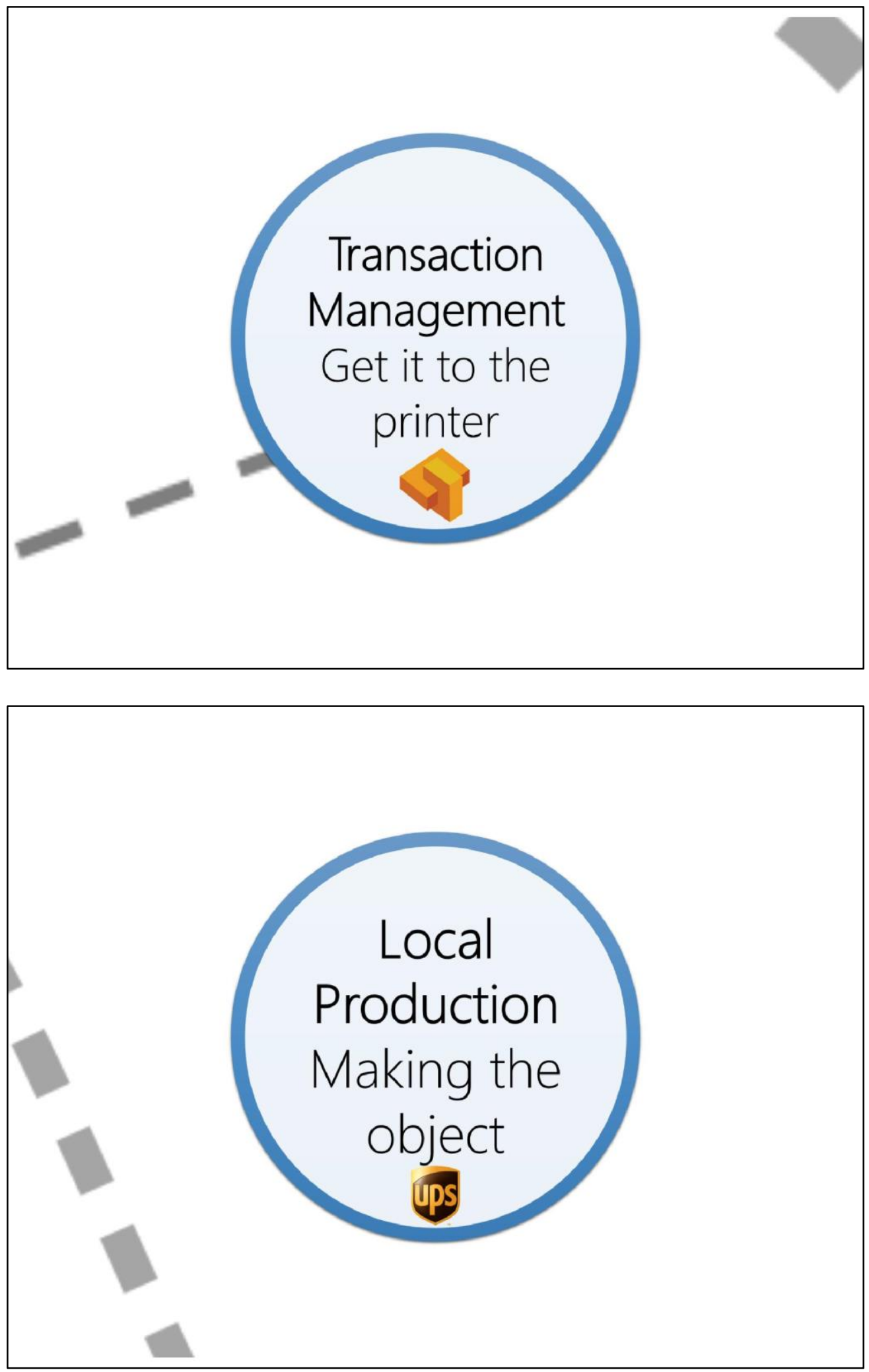

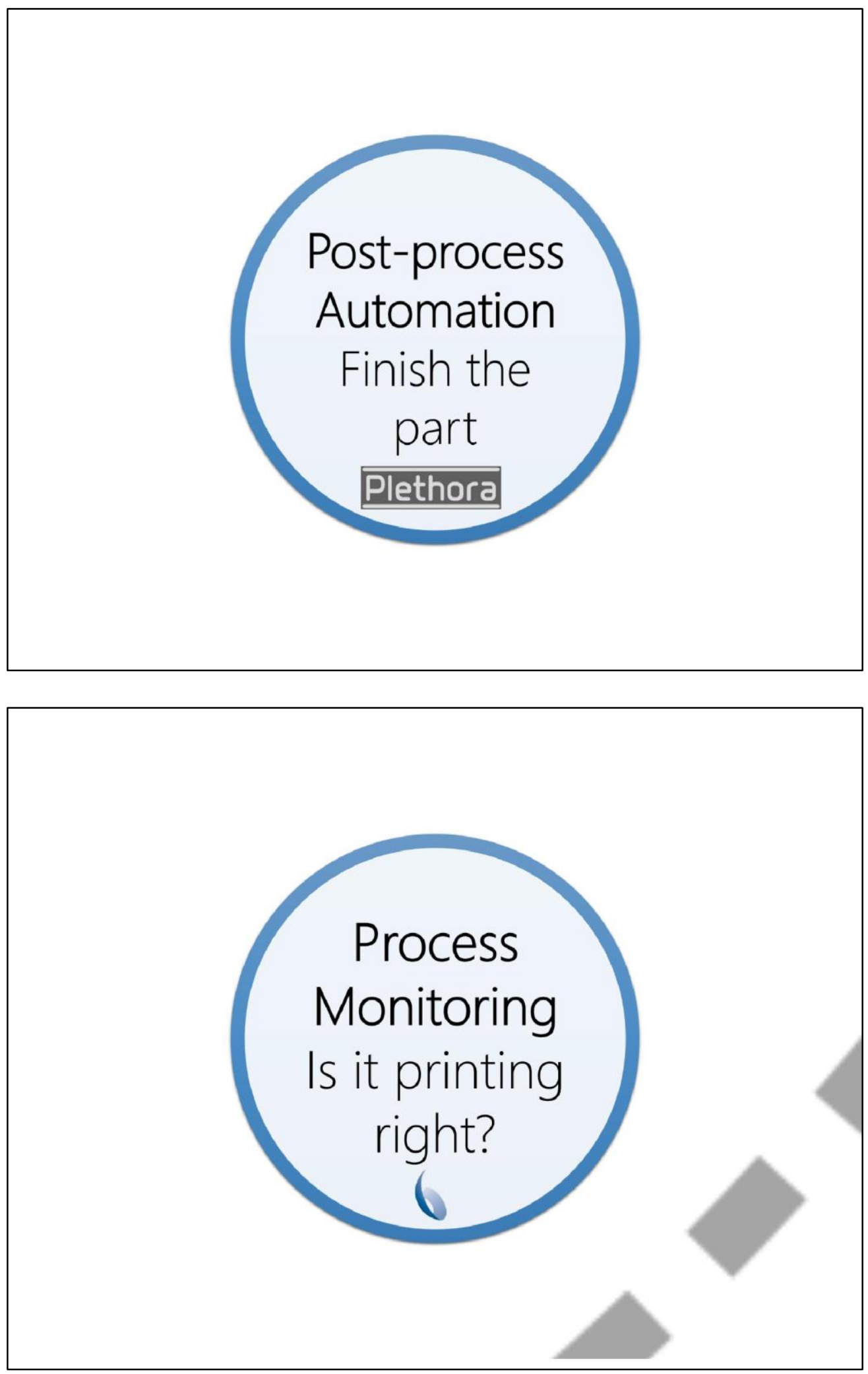

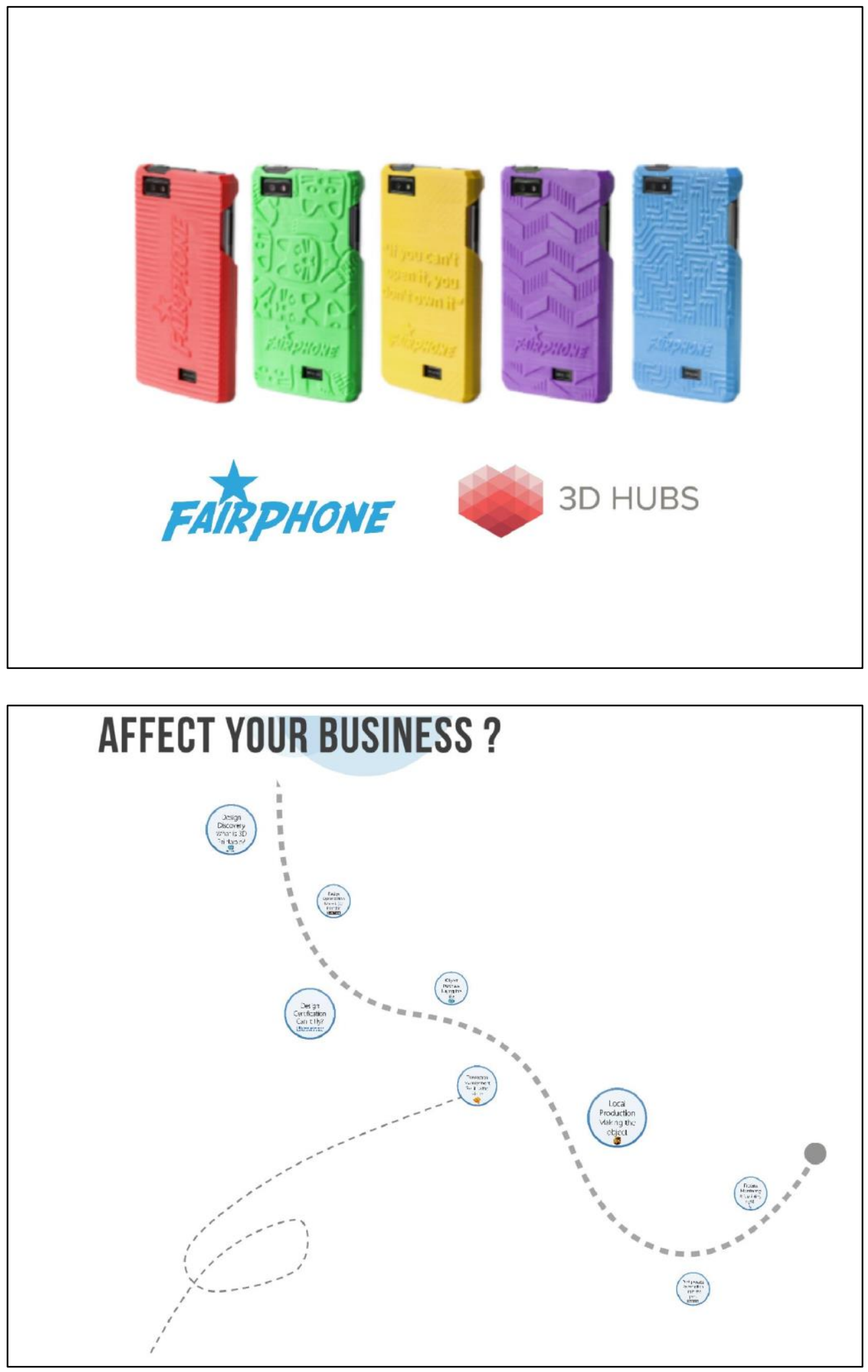

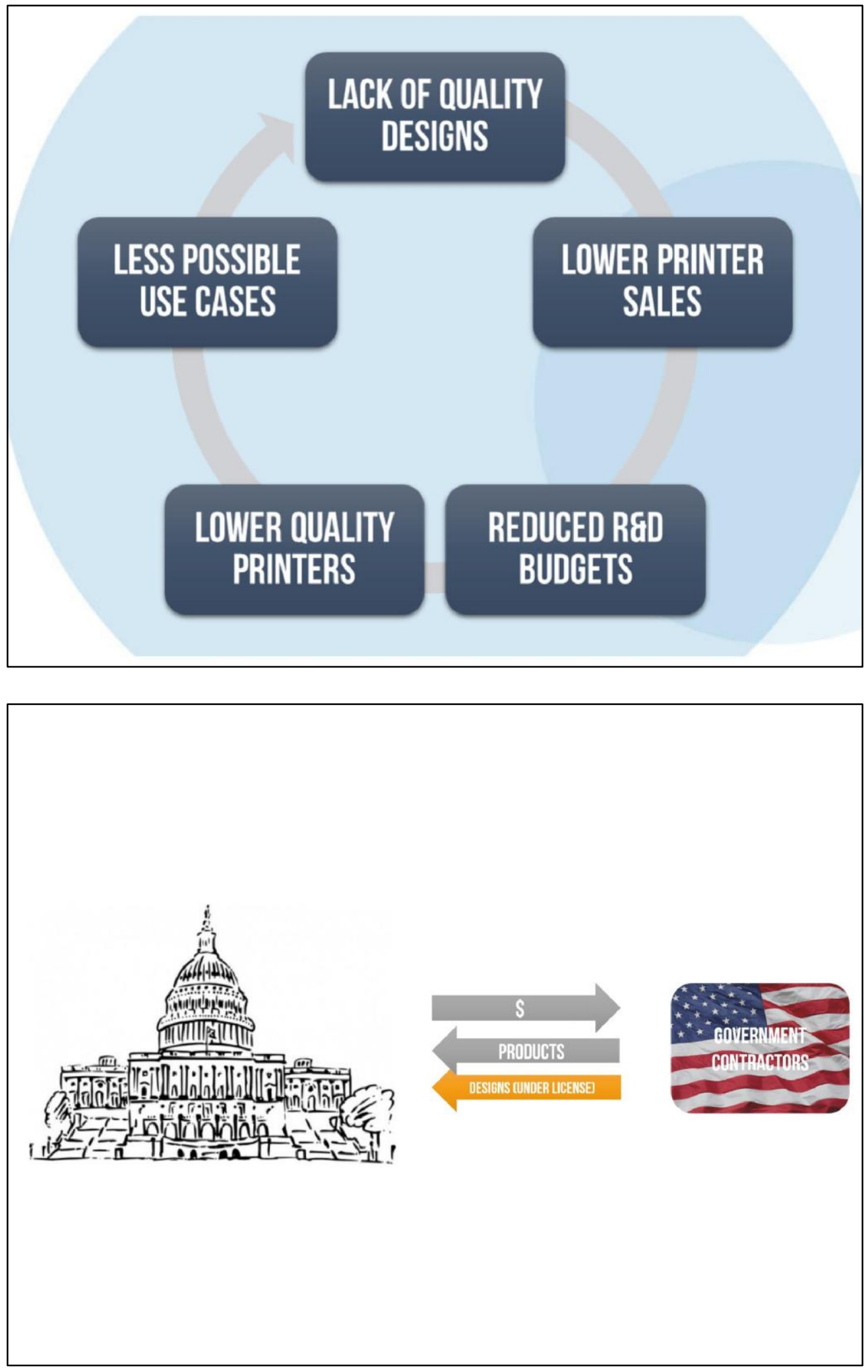


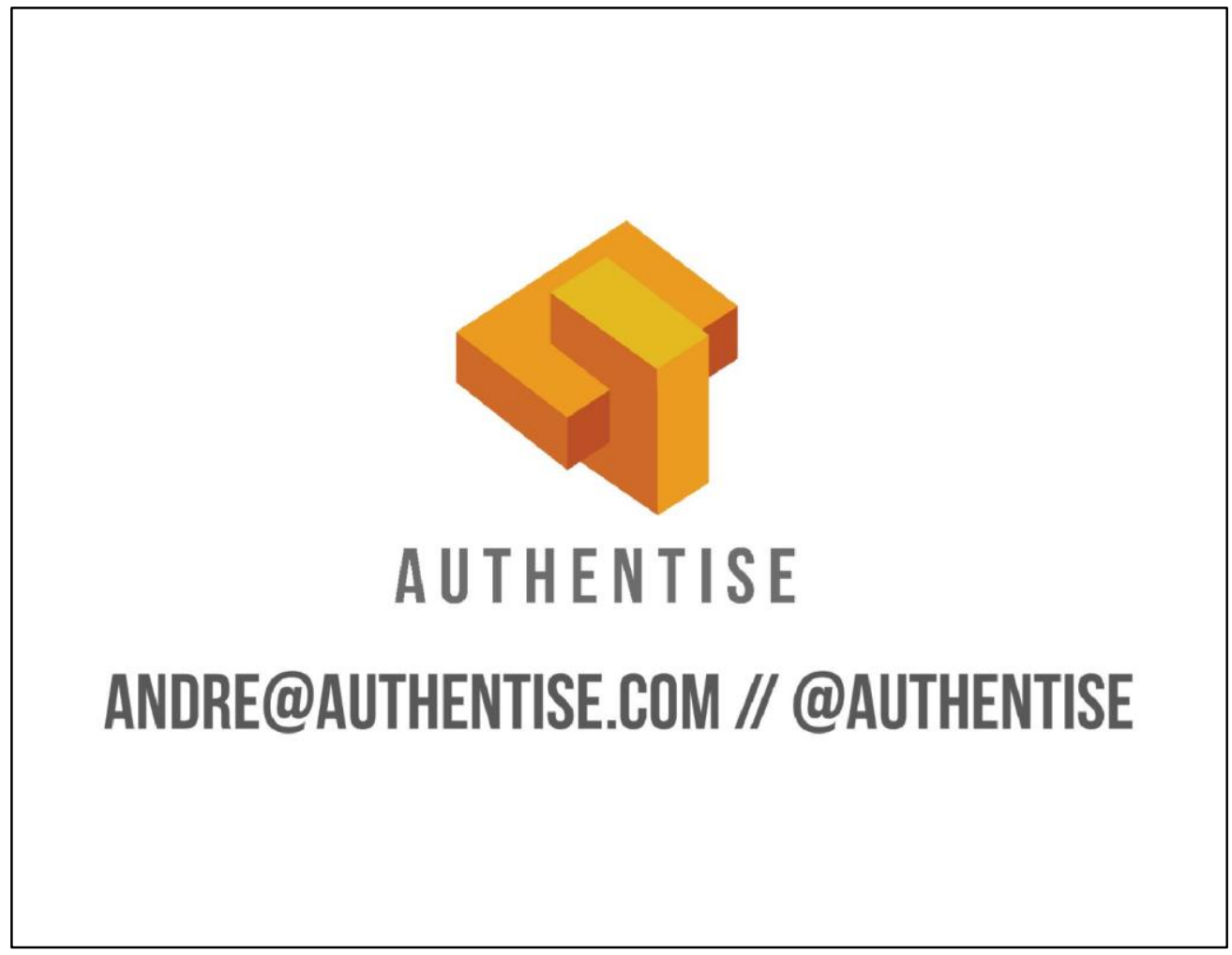




\section{$3 \quad$ Summary of Attendee Perceptions}

This section summarizes attendee perceptions as gathered throughout the symposium, including during presentations and through information gathering exercises. At the start of the symposium, attendees were asked to anonymously list as many thoughts / items as they could under each of the following categories:

- Risks;

- Challenges;

- Existing Solutions, and

- Potential / Theoretical Solutions.

20 percent of attendees submitted their responses, listed in Appendix A.

In addition, during the closing session, attendees were asked to identify thoughts / items under the following categories:

- Standards;

- Guidance;

- Tools, and

- Gaps.

The responses from this exercise are listed in Appendix B.

Several attendees identified culture / humans as a significant risk or challenge to the cybersecurity of DDM, and to cybersecurity in general. Cybersecurity education at all levels of a manufacturing organization was desired. Changing the priorities and culture of manufacturing organizations is challenging due to a lack of understanding of cybersecurity risks and benefits. Business cases or examples were desired. A few attendees mentioned legal requirements as a potential solution and there were a few comments questioning who bears the burden of the risk of an attack - the IP owners, the vendor(s), or the government.

Threats to the integrity of designs and systems were a common thread in responses. Some mentioned confidentiality of intellectual property as a concern and only a few identified availability concerns. Software vulnerabilities were called out a few times, but most responses focused on the final product. The nature of the digital supply chain was identified several times as a challenge with attendees specifically calling out the volume and types of data to be protected in a distributed and open manufacturing environment.

Quality control and event detection capabilities were desired. A few attendees mentioned the use of encryption throughout the manufacturing process as a potential solution. Other potential / desired technical capabilities identified by respondents included: distributed network security solutions, authentication mechanisms, automated and real-time monitoring and control, embedded security solutions, and residual data removal tools. It was stressed in responses and throughout the symposium that any technical solution must be simple and easy and preferably all-encompassing- "an easy button". 
Another common thread in responses was the suggestion for guidelines specific to DDM based on NIST SP 800-53 [1], the NIST Cybersecurity Framework [2], existing ISO standards, and industry best practices. Technical standards, such as protocols and formats, were also mentioned by several as representing a gap, or opportunity, for improving cybersecurity. Attendees provided the following list of standards and guidelines as providing a potential foundation for future DDM-specific cybersecurity standards and guidelines.

- IEC 62264-1:2013 - Enterprise-control system integration -- Part 1: Models and terminology [4]

- ISA-95, Enterprise-Control System Integration [5]

- ISO / ASTM52915 - 13, Standard Specification for Additive Manufacturing File Format (AMF) Version $1.1[3]$

- ISO 10303 -242:2014, , Industrial automation systems and integration -- Product data representation and exchange -- Part 242: Application protocol: Managed model-based $3 D$ engineering [6]

- ISO 14306:2012, Industrial automation systems and integration -- JT file format specification for $3 D$ visualization [7]

- ISO 14739-1:2014, Document management -- 3D use of Product Representation Compact (PRC) format -- Part 1: PRC 10001 [8]

- ISO/IEC 27000:2014, Information technology -- Security techniques -- Information security management systems -- Overview and vocabulary [9]

- NAS 9924, Cybersecurity Baseline [10]

- NIACAP-DIACAP (now obsolete, see DoDI 8510.01 and [11] CNSSP No. 22[12])

- NIST Framework for Improving Critical Infrastructure Cybersecurity [2]

- $\quad$ NIST IR 8023, Risk Management for Replication Devices [13]

- NIST SP 800-53 Revision 4, Security and Privacy Controls for Federal Information Systems and Organizations [1]

- $\quad$ NIST SP 800-82, Revision 2, Guide to Industrial Control Systems (ICS) Security [14] 


\section{Conclusions}

Direct Digital Manufacturing is poised to revolutionize the manufacturing industry. A collaborative public and private approach is necessary to improving the cybersecurity of DDM processes and technology. This symposium was intended to be a step in that direction.

Although the presenters were from diverse backgrounds representing a variety of viewpoints, each made similar points:

- Cybersecurity risks to DDM are very real;

- Cybersecurity threats are the Achilles heel of the current manufacturing revolution;

- There is a real opportunity to make the manufacturing supply chain more secure than it has ever been, and

- The time to build cybersecurity in to the DDM process is now.

Attendees identified several risks and opportunities for building cybersecurity into DDM. Many attendees identified the integrity of designs and machines as a major risk while a few also mentioned intellectual property concerns. Gaps and potential solutions were grouped into four categories:

- Education / awareness of risks and cost/benefits;

- Technical solutions such as encryption capabilities and network monitoring;

- Technical standards such as a security option in existing standard file formats, and

- Guidance / best practice documents based on existing NIST publications.

With its expertise in advanced manufacturing and information technology, NIST is well poised to address these concerns. The NIST ITL has already developed cybersecurity guidance related to Cyber Physical Systems and Industrial Control Systems. There is an opportunity to include DDM cybersecurity considerations into future revisions of existing programs and publications. Also, the National Initiative for Cybersecurity Education (NICE) has begun to look at how to help manufacturers be more aware of cybersecurity risks that they may not have recognized. Additionally, the National Cybersecurity Center of Excellence (NCCoE) uses existing standards and technology to architect solutions to difficult cybersecurity problems and DDM may be a candidate. Results from this symposium will help guide future efforts in these areas. 


\section{Appendix A: Response Sheet Results}

At the beginning of the symposium, attendees were asked to list as many items/thoughts as they could under the following categories: Risks, Challenges, Existing Solutions, and Potential / Theoretical Solutions. Attendees were not limited as to the scope of their responses and encouraged to write whatever came to mind. The following is a compilation of the responses received in each category. Responses are listed in alphabetical order and were transcribed as closely as possible, including grammar, abbreviations, and spelling. References have been added where possible and are included in Appendix F. An analysis of the responses, along with responses in Appendix B, is provided in section 3 of this publication.

Risks:

- Altering data to change specs of finish products

- Availability

- Components in sensitive applications may have unintended / undesirable performance characteristics that are undetectable

- Confidentiality

- Corruption of imbedded software @ machine

- Corruption of STL files

- Damage of manufacturing equipment

- Design tools vulnerability -> CAD \& pre-cad part of

- Detection of inherent flaw - pilphereal IP is analyzed for existing flaw

- Ensure the small \& medium enterprise have the tools are reasonable price point

- EtherCat or Industrial IP security?

- Getting tools to the right level capability at right price / affordable

- Government entities each seem to have their own program for cyber security. The risk is two-fold: (1) they are talking, but not WORKING together. Wasting resources and efforts (2) Government is way behind industry, and not bringing them in to address this substantial gap

- Integrity

- IT/OT convergence --> how do I secure this... ...

- IVV of file transport from central storage to production facility

- Modification of model

- OEMs not ensuring security (\& keeping backdoors open for "maintenance")

- Tainted products (additional functionality)

- The human aspect (social engineering)

- Theft of intell property

- Theft of IP

- Treats to RF specturm - wireless is increasing the comms component of choice on factory floor. 802.3, 802.11ad etc

- Understanding 3D printer, direct digital in the context of 3D phenomenon [i.e. same files could be used for manufacturing or decision support

- Uneducated workforce 
- Un-maintained manufacturing equipment (outdated OS, virus definitions, firewall, firmware, etc.)

Challenges:

- 3D, HD, FMV

- Automated

- Automation security

- Balancing benefits of open-source / open architecture machines \& file formats with dangers of cyber vulnerabilities

- Digital rights management / digital asset management

- Digital supply chain

- DISTRIBUTED manufacturing --> factory to factory

- eCommerce --> Will be part of the supply chain and will provide its own set of challenges

- Educating workforce about cyber-physical concerns

- Embedded system / PLC, SCADA, ACS security

- Front end costs of cyber controls are hard to justify

- Having the right folks be the custodian of data / system

- How to capture design intent for validation / certification

- Integration of various data warehouse within enterprise that have to interface with each other (i.e. PDM/PLM, MRP/ERP/MES and Accounting/HR) to provide the integrity, availability, \& confidentiality

- Intellectual property management

- Lack of business case

- Manufacturing systems are not often updated (patches, firmware, more IT functionality than needed)

- Mfg culture, gap to IT culture

- Modeling and simulation precursor to decision to manufacture and design for manufacturing

- MOM [Manufacturing Operations Management Security]

- Organizational change management

- PCII - protected critical infrastructure Information

- PMI - production Manufacturing Information

- Poor acquisition policy that doesn't drive security

- Poor secure engineering design techniques (hardware \& software)

- Prioritization of what is really important

- Quality control of microarchitecture

- Real-Time systems (synchrophasor, EtherCAT, etc)

- Role based access for M2M (machine to machine) exchanges

- Security as a requirement for the PLC and PLC of infrastructure and PLC of FW/SW

- Sensor network security

- The value proposition

- Trust 
- Understand tools, techniques and processes to protect fidelity from design thru production - what tool, at what cost, at what reduction in efficiency

- Volume of 3D digital media

Existing Solutions

- 5 layer manufacturing protocol stack $[5,4]$

- Encryption

- Fundamental best practices are available in 800 series SPs and some contemporary IT security publications

- NIST framework is good starting point

- Training / awareness

- Use of existing protocol for traditional manufacturing

Potential / Theoretical Solutions

- 20 Critical controls for manufacturers

- Anecdotal

- Benchmark DoD/DOE defense contractors for best practices

- Content distribution networks - edge computing security

- Encrypt lifecycle

- Encrypted streaming

- Factory of the future dialog

- Focus on model based ecosystems: provides an architecture and governance

- IACAP-DIACAP - 800-134 (guess at \#) - DoD continues to evolve "mandatory" standard

- Increased use of encryption

- Need a single entity that government can use to advance itself in this area. To succeed needs non-government owner who can bring all gov. entities together pooling resources, and incorporate industry to get current best practices. Suggest DMDLL as they are already doing a project on this involving government and industry. Possibly a more comprehensive follow on project

- NEED AN EASY button for manufacturing floor

- NTSB and auto - safety - manufacturers are responsible for standards - policy and law follow recommendation but a federal law was necessary to institute the mandate for commercial sector

- Standards are probably the best way of balancing concerns of vulnerabilities, openness, \& privacy ( $\&$ business)

- What risk reduction strategies, tools, and solutions exist? - A primer for manufacturing would be great!! Perhaps a good project for NAMII?

- When we take people out of the loop a lot of vulnerabilities go away 


\section{Appendix B: Working Session Results}

During the working session, attendees were asked to identify any standards, guides, or tools that could be applied to cybersecurity in DDM. They were also asked to identify any gaps in those areas, or anything that was missed during the symposium. Attendees were not limited as to the scope of their responses. The following is a compilation of the responses received in each category. Responses are listed in alphabetical order and were transcribed as closely as possible, including grammar, abbreviations, and spelling. References have been added where possible. An analysis of the responses, along with responses in Appendix A, is provided in section 3 of this publication.

Standards:

- $\quad \mathrm{AMF} \&$ ISO JT [7]

- IEC [16]

- IEEE [17]

- ISO ? Dealing with PDF / PRC format [8]

- ISO [18]

- ISO 10303 AP 242 [6]

- $\quad$ ISO 27000 [9]

- ITSI

- NAS 9924 [10]

- National Aerospace STDs published by Aerospace Industries Assoc. www.aia-nas.org [19]

- NIST 800-53 [1]

- Sector SIGs

- Security Spec for ISO AMF standard [3]

- See references to draft CPS PWG working group report [20]

- $\quad$ Step 242 [6]

\section{Guides:}

- Cyber awareness for the shop floor

- NIST SP 800-82 [14]

- Overlay for 800-53 [1] is important in bridging IT to OT thinking

- Risk Management adapted for DDM

Tools:

- DEA tools

- NICE [21]

- Residual data removal tool

- Threat data sharing mechanisms 
Gaps:

- Authentication of Articles Connected to IoT

- Awareness of costs associated with NOT integrating security

- Breach Disclosure

- Drivers for secure hardware \& software design

- Encryption approaches

- FBI is an active player in cybersecurity

- Flaw hack marketplace

- Formats

- Integration approaches

- International laws and agreement to prosecute the sources of cybersecurity event and bad actors

- Manufacturing Protocol Stack (Purdue) [15]

- Material quality standards - powder (distribution, properties), polymer

- NEED a guide for Business Case Analysis (for cybersecurity in mfg); NEED data/case examples to support

- Rule of unfettered Innovation / open software mode

- Transport protocols

- Who owns the problem

- Who owns what? - IP ownership

- Who will own the solution - if industry doesn't does it roll over to government 


\section{Appendix C: Speaker Biographies}

The following are speaker biographies as included in the agenda for the symposium, in presentation order.

\section{Michael F. Molnar}

Director, NIST Advanced Manufacturing Program Office

Director, Advanced Manufacturing National Program Office (AMNPO)

Mike Molnar likes to be introduced simply as "a manufacturing guy from industry" with nearly 30 years of experience in advanced manufacturing. To help provide an industry focus in 2011 he was named the first Chief Manufacturing Officer of the National Institute of Standards and Technology. Today Mike leads the NIST Advanced Manufacturing Program Office for extramural manufacturing programs and also serves as the director of the interagency Advanced Manufacturing National Program Office. As called for by the Advanced Manufacturing Partnership initiative, the AMNPO's mission is to foster industry-led partnerships and to form a "whole of government" approach to strengthen competitiveness and innovation in U.S. manufacturing.

Mike's experience includes leadership roles in advanced manufacturing, metrology, manufacturing systems, quality, technology development, sustainability and industrial energy efficiency. His credentials include service as a Federal Fellow in the White House Office of Science and Technology Policy, and election as Fellow of both the American Society of Mechanical Engineers and the Society of Manufacturing Engineers. He is a licensed Professional Engineer, a Certified Manufacturing Engineer and a Certified Energy Manager. He received a Master of Business Administration from the University of Notre Dame, and both a Master of Science in Manufacturing Systems Engineering and a Bachelor of Science in Mechanical Engineering from the University of Wisconsin. He is an active member of professional societies, consortia and volunteer organizations.

\section{Christopher B. Williams}

Associate Professor, Virginia Tech Department of Mechanical Engineering

Christopher B. Williams is an Associate Professor with a joint appointment with the Department of Mechanical Engineering and the Department of Engineering Education at Virginia Tech. He is the Director of the Design, Research, and Education for Additive Manufacturing Systems (DREAMS) Laboratory and Associate Director of the Macromolecules \& Interfaces Institute. His research contributions have been recognized by six Best Paper awards at international design, manufacturing, and engineering education conferences. He is a recipient of a National Science Foundation CAREER Award (2013), the 2012 International Outstanding Young Researcher in Freeform and Additive Fabrication Award, and the 2010 Emerald Engineering Additive Manufacturing Outstanding Doctoral Research Award. Chris holds a Ph.D. and M.S. in Mechanical Engineering from the Georgia Institute of Technology (Atlanta, Georgia) and a B.S. with High Honors in Mechanical Engineering from the University of Florida (Gainesville, Florida). 
Scott Zimmerman CISSP-ISSEP

Principal IT Advisor, Concurrent Technologies Corporation (CTC)

\section{Dominick Glavach CISSP, GCIH}

Principle Fellow, Information Systems Security Engineer, CTC

Scott Zimmerman, CISSP-ISSEP is a Principal Technical Advisor at Concurrent Technologies Corporation with 20 plus years of Cyber Security experience. Mr. Zimmerman specialized expertise includes cyber security, cloud/mobile computing and systems engineering. Mr. Zimmerman's education includes a BS in Management Information Systems and AS in Electronic/Computer Technology. He is a Certified Information Systems Security Professional (CISSP); Information Systems Security Engineering Professional (ISSEP).

Mr. Glavach is a Principle Information Systems (IS) Security Engineer and CISO at Concurrent Technologies Corporation (CTC). He serves as the Cyber Security technical lead in CTC's Enterprise Infrastructure, provides CTC's clients with Cyber technical leadership and Subject Matter Expertise (SME). Mr. Glavach received his BS in Computer Science from the Indiana University of Pennsylvania, is a Certified Information System Security Professional (CISSP), an active member of the Information Assurance Technology Analysis Center SME Program and member of the Cloud Security Alliance (CSA).

The speakers specialize in cyber attack methods, attack warning and detection, and cyber countermeasures. They have presented numerous talks on cloud forensics, cyber adversaries and advanced persistent threats to a wide range of public and government audiences.

Concurrent Technologies Corporation (CTC) is an independent, nonprofit, applied scientific research and development professional services organization providing innovative management and technology-based solutions to government and industry. Established in 1987, CTC operates from more than 50 locations with a staff of over 1,400 employees. As a nonprofit 501(c)(3) organization, CTC's primary purpose and programs are to undertake applied scientific research and development activities that serve the public interest. We conduct impartial, in-depth assessments and technical evaluations that emphasize increased quality, enhanced effectiveness, and rapid technology transition and deployment. CTC offers a broad range of services and capabilities, coupled with real-world experience. For more information about CTC, visit WwW.ctc.com.

\section{Dr. Michael McGrath \\ NDIA Manufacturing Division}

Michael McGrath is an independent consultant who provides analytic support for government and industry technology programs. He is also a Senior Technical Advisor (and former Vice President) at Analytic Services Inc. (ANSER), a not-for-profit government services organization. He previously served as the Deputy Assistant Secretary of the Navy for Research, Development, Test and Evaluation (DASN(RDT\&E)), where he was a strong proponent for improvements in technology transition, modeling and simulation, and test and evaluation. In prior positions, he 
served as Vice President for Government Business at the Sarnoff Corporation, ADUSD for Dual Use and Commercial Programs in the Office of the Secretary of Defense (OSD), Assistant Director for Manufacturing at the Defense Systems Research Projects Agency (DARPA-DSO), and Director of the DoD Computer-aided Acquisition and Logistics Support (CALS) program. While at DARPA, he managed the Affordable Multi-Missile Manufacturing Program and the Agile Manufacturing program. He was also heavily involved in DARPA's dual-use Technology Reinvestment Project and has been a strong advocate for defense use of commercial technology advances. His early government career included positions in Logistics Management at Naval Air Systems Command and in Acquisition Management in OSD. He is a Senior Fellow at the Potomac Institute for Policy Studies, a director of South Carolina Research Authority Applied R\&D, and a member of the National Research Council's Materials and Manufacturing Board, the Defense Materials, Manufacturing and Infrastructure Committee (chair), the Penn State ARL Materials and Manufacturing Advisory Board, and the Georgia Tech Manufacturing Institute Advisory Board.

Dr. McGrath holds a BS in Space Science and Applied Physics and an MS in Aerospace Engineering from Catholic University, and a doctorate in Operations Research from George Washington University.

\section{Robert Zollo}

President, Avante Technology, LLC

Mr. Zollo is President and Founder of Avante Technology, LLC, a privately held company that develops, markets and licenses advanced 3D printing technology to 3D printer OEM, manufacturers and engineering firms. Prior to that he was President and Founder of Software Architects, Inc. a developer of electronic systems for OEM in a variety of industries, including 3D printing, digital imaging and optical recording. As Chairman of the Optical Storage Technology Association, Mr. Zollo was responsible for the development of ISO 13346, the international standard that defines the digital file format used in all DVD's, Blu-ray discs, CAT scan, MRI and digital X-ray systems. He also led the development of four patents relating to digital file management, image manipulation and file interoperability, and is the inventor of a patent pending method for controlling the printing of new engineering grade composite materials in FDM printers. Mr. Zollo holds a Bachelor of Science degree in Engineering from the U.S. Military Academy at West Point, an MBA from Southern Illinois University and conducted his graduate technical studies at the University of Southern California's school of engineering. $\mathrm{He}$ is currently working on enhancements to the new ISO AMF standard defining the 3D file description language for additive manufacturing applications.

\section{Dr. Claire Vishik}

Trust and Security Technology and Policy Director, Intel Corporation 
Dr. Claire Vishik's work at Intel Corporation focuses on hardware security, Trusted Computing, privacy enhancing technologies, some aspects of cryptography and related policy issues. Claire is a member of the Permanent Stakeholders Group (Advisory Board) of ENISA, the European Network and Information Security Agency. She is an advisor to a number of cybersecurity R\&D and policy projects, initiatives, and organizations, including the cryptography program at the University of Bristol or Oxford Cybersecurity Center for Capacity Building and is on the leadership teams of several organizations and initiatives tasked with the development of R\&D strategies in cybersecurity in the US, Europe, and beyond. Claire is active in standards development and is on the Board of Directors of the Trusted Computing Group and on the Council of the Information Security Forum. Claire received her PhD from the University of Texas at Austin. Prior to joining Intel, Claire worked at Schlumberger Laboratory for Computer Science and AT\&T Laboratories. Claire is the author of numerous papers and reports and an inventor on $30+$ pending and granted U.S. patents.

\section{Andre Wegner}

Co-founder \& CEO, Authentise

Andre Wegner is co-founder and CEO of Authentise (www.authentise.com), the licensing and services platform for Distributed Manufacturing. Authentise secure streaming and quality assurance technology for 3D printing enables design owners to share their digital manufacturing designs with confidence, and get paid per print. Authentise Consulting also assists Fortune 100 corporations put 3D printing at the heart for their business. He is a frequent speaker on emerging intellectual property issues in 3D Printing and opportunities of distributed manufacturing at events such as Singularity University, Rapid, Designer of Things, Inside 3D Printing, 3D Print Show, Pacific Crest \& WIRED. He has been quoted in publications such as BBC News, MIT Tech Review, Chicago Tribune, and Bloomberg. Prior to founding Authentise he managed a venture capital fund in Nigeria and advisory services in India. He is a graduate of St. Andrews University (UK), ESSEC (France) and Singularity University (California). 


\section{Appendix D: Attendees List}

\begin{tabular}{|c|c|}
\hline Registrant Name & Organization \\
\hline Clara Asmail & NIST MEP \\
\hline Lawrence Balash & Nova Corporation \\
\hline David Barrett & Department Of Navy-Chief Of Naval Operations \\
\hline Dean Bartles & UI Labs \\
\hline Michelle Bezdecny & Anser - OSD/Mantech \\
\hline Allen Egon Cholakian & IRDFproject Harvard / Columbia \\
\hline Bill Coccoli & NGC \\
\hline Thomas Conkle & G2, Inc. \\
\hline Khershed Cooper & NSF \\
\hline Charles Crum & Office Of Inspector General, Us Postal Service \\
\hline Nicholas Deliman & MDA Information Systems \\
\hline \multicolumn{2}{|l|}{ Tuong-Vy Do } \\
\hline Gavin Garner & University Of Virginia \\
\hline \multicolumn{2}{|l|}{ Dom Glavach } \\
\hline Daniel Green & Space And Naval Warfare Systems Command \\
\hline Ryan Hayleck & NAVSEA \\
\hline Paul Huang & NIST \\
\hline Brian Hubbard & G2, Inc. \\
\hline \multicolumn{2}{|l|}{ Michele Hughes } \\
\hline Lawrence John & Analytic Services Inc. \\
\hline Waide Jones & Lockheed Martin \\
\hline Ben Kassel & Naval Sea Systems Command \\
\hline Bruce Kramer & NSF \\
\hline Francis Lee & Howard County Public School Systems \\
\hline Michael Mcgrath & Analytic Services Inc (Anser) \\
\hline Mike Molnar & NIST \\
\hline Ed Morris & NCDMM \\
\hline Wesley Old Coyote & State Of Montana \\
\hline Yaowe Ong & CSC \\
\hline
\end{tabular}




\begin{tabular}{|c|c|}
\hline Celia Paulsen & NIST \\
\hline Al Payne & Theta Solutions \\
\hline Paul Petronelli & Palm Associates, Inc. \\
\hline James Rentsch & Aerospace Industries Association \\
\hline Chris Root & NAVAIR Fleet Readiness Center Southwest \\
\hline Scott Storms & NAVSSES \\
\hline Rebecca Taylor & NCMS \\
\hline Joe Veranese & NCDMM \\
\hline Patrick Violante & NAVSSES \\
\hline Claire Vishik & Intel \\
\hline R Wachter & \\
\hline Andre Wegner & Authentise Inc \\
\hline Eric Wilcox & SAIC \\
\hline Craig Young & DDC-ITS \\
\hline Scott Zimmerman & CTC \\
\hline Robert Zollo & Avante Technology, Llc \\
\hline
\end{tabular}




\section{Appendix E: Acronyms}

ACS

AM

$\mathrm{AMF}$

CAD

DDM

DEA

DMDII

DoD

DOE

ERP

FMV

FW

HD

IoT

IP

ISO

IT

IVV

MES

MOM

MRP

NAMII

NIST

NICE

NNMI

NTSB

OEM

OS
Access Control System

Additive Manufacturing

Additive Manufacturing File Format

Computer Aided Design

Direct Digital Manufacturing

Data envelopment analysis

Digital Manufacturing and Design Innovation Institute

Department of Defense

Department of Energy

Enterprise resource planning

Full Motion Video

Firmware

High Definition

Internet of Things

Intellectual Property

International Organization for Standardization

Information Technology

Independent Verification and Validation

Manufacturing Execution System

Manufacturing Operations Management

Material requirements planning

National Additive Manufacturing Innovation Institute

National Institute of Standards and Development

National Initiative for Cybersecurity Education

National Network for Manufacturing Innovation

National Transportation Safety Board

Original Equipment Manufacturer

Operating System 


$\begin{array}{ll}\text { OT } & \text { Operations/Operational Technology } \\ \text { PCII } & \text { Protected Critical Infrastructure Information } \\ \text { PDM } & \text { Product data management } \\ \text { PLC } & \text { Programmable Logic Controller } \\ \text { PLM } & \text { Product Lifecycle Management } \\ \text { PMI } & \text { Production Manufacturing Information } \\ \text { RF } & \text { Radio Frequency } \\ \text { SCADA } & \text { Supervisory Control and Data Acquisition } \\ \text { SIG } & \text { Special Interest Group } \\ \text { STD } & \text { Standard } \\ \text { STL } & \text { Stereolithography } \\ \text { SW } & \text { Software }\end{array}$




\section{Appendix F: References}

[1] NIST Special Publication (SP) 800-53 Revision 4, Security and Privacy Controls for Federal Information Systems and Organizations, Gaithersburg, Maryland, 2013, http://dx.doi.org/10.6028/NIST.SP.800-53r4

[2] Cybersecurity Framework, National Institute of Standards and Technology, http://www.nist.gov/cyberframework/, 2014

[3] ISO / ASTM52915 - 13, Standard Specification for Additive Manufacturing File Format (AMF) Version 1.1, Astm, 2013, http://www.astm.org/Standards/ISOASTM52915.htm

[4] IEC 62264-1:2013, Enterprise-control system integration -- Part 1: Models and terminology, International Organization for Standardization, 2013, http://www.iso.org/iso/catalogue_detail.htm?csnumber $=57308$

[5] ISA-95, Enterprise-Control System Integration, International Society of Automation, https://www.isa.org/isa95/

[6] ISO 10303-242:2014, Industrial automation systems and integration -- Product data representation and exchange -- Part 242: Application protocol: Managed model-based $3 D$ engineering, International Organization for Standardization, 2014, http://www.iso.org/iso/iso_catalogue/catalogue_tc/catalogue_detail.htm?csnumber=5762 $\underline{0}$

[7] ISO 14306:2012, Industrial automation systems and integration -- JT file format specification for 3D visualization, International Organization for Standardization,2012, http://www.iso.org/iso/catalogue_detail.htm?csnumber=60572

[8] ISO 14739-1:2014, Document management -- 3D use of Product Representation Compact (PRC) format -- Part 1: PRC 10001, International Organization for Standardization, 2014, http://www.iso.org/iso/catalogue_detail.htm?csnumber=54948

[9] ISO/IEC 27000:2014, Information technology -- Security techniques -- Information security management systems -- Overview and vocabulary, International Organization for Standardization, 2014, http://www.iso.org/iso/catalogue_detail?csnumber=63411

[10] NAS9924, Cybersecurity Baseline, Aerospace Industries Association,2013, https://global.ihs.com/doc_detail.cfm?\&rid=AIA\&input_doc_number=NAS\%209924\%2 
CNA\&item_s_key $=00601403 \&$ item key_date $=861003 \&$ input_doc number $=N A S \% 209$ 924\%2CNA\&input_doc_title=\#abstract

[11] Department of Defense Instruction (DoDI) 8510.01, Risk Management Framework (RMF) for DoD Information Technology (IT), Department of Defense, 2014, http://www.dtic.mil/whs/directives/corres/pdf/851001_2014.pdf

[12] Policy on Information Assurance Risk Management for National Security Systems, Committee on National Security Systems (CNSS), CNSSP No. 22, 2012, http://www.ncix.gov/publications/policy/docs/CNSSP_22.pdf

[13] Dempsey, Kelley and Paulsen, Celia. NIST Internal Report (IR) 8023, Risk Management for Replication Devices, National Institute of Standards and Technology, 2015, http://dx.doi.org/10.6028/NIST.IR.8023

[14] NIST Special Publication (SP) 800-82 Revision 2, Guide to Industrial Control Systems (ICS) Security, second public draft, National Institute of Standards and Technology, Gaithersburg, Maryland, 2008, http://csrc.nist.gov/publications/drafts/800$\underline{82 \text { r2/sp800_82_r2_second_draft.pdf }}$

[15] Williams, Theodore J. "The Purdue Enterprise Reference Architecture", Computers in Industry, 24 (1994), pp. 141-158, http://dx.doi.org/10.1016/0166-3615(94)90017-5.

[16] International Electrotechnical Commission (IEC), http://www.iec.ch/, 2015

[17] IEEE, https://www.ieee.org/index.html, 2015

[18] ISO - International Organization for Standardization, http://www.iso.org/iso/home.html, 2015

[19] Aerospace Industries Association, National Aerospace Standards Aerospace Industries Association, http://www.aia-aerospace.org/national_aerospace_standards/, 2015

[20] Cyber-Physical Systems Public Working Group, http://www.cpspwg.org/, 2015

[21] The National Initiative for Cybersecurity Education (NICE), National Institute of Standards and Technology, http://csrc.nist.gov/nice/, 2015 